\title{
Effective fractal dimension: foundations and applications
}

\author{
by \\ John M. Hitchcock \\ A dissertation submitted to the graduate faculty \\ in partial fulfillment of the requirements for the degree of \\ DOCTOR OF PHILOSOPHY
}

\author{
Major: Computer Science \\ Program of Study Committee: \\ Jack H. Lutz, Major Professor \\ Pavan Aduri \\ Clifford Bergman \\ Soumendra N. Lahiri \\ Giora Slutzki
}

Iowa State University

Ames, Iowa

2003

Copyright (C) John M. Hitchcock, 2003. All rights reserved. 


\section{Graduate College}

Iowa State University

This is to certify that the doctoral dissertation of

John M. Hitchcock

has met the dissertation requirements of Iowa State University

Major Professor

For the Major Program 


\section{TABLE OF CONTENTS}

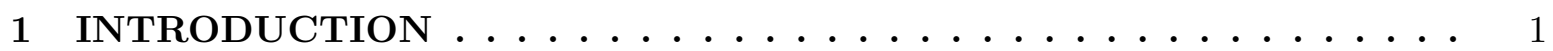

1.1 Foundations of Effective Fractal Dimension . . . . . . . . . . . . . . . 3

1.1.1 Effective Strong Dimension . . . . . . . . . . . . . . . 4

1.1.2 Gales Suffice for Constructive Dimension . . . . . . . . . . . . . 5

1.1.3 Entropy Rates and Kolmogorov Complexity . . . . . . . . . . . 5

1.1.4 Log-Loss Unpredictability . . . . . . . . . . . . . . . . 6

1.2 The Arithmetical Hierarchy . . . . . . . . . . . . . . . . . . 6

1.2.1 Correspondence Principles . . . . . . . . . . . . . 6

1.2.2 Complexity of Dimension Classes . . . . . . . . . . . . . . . . 7

1.3 Polynomial-Time Reductions . . . . . . . . . . . . . . 8

1.3.1 Small Spans . . . . . . . . . . . . . . . . 8

1.3.2 Degrees of Arbitrary Dimensions _ . . . . . . . . . . . . . . 9

1.3.3 Reductions to Nondense Languages . . . . . . . . . . . . . . . . . 9

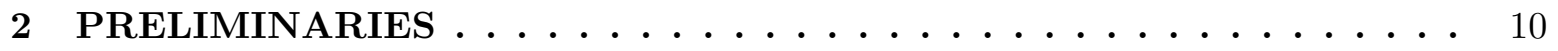

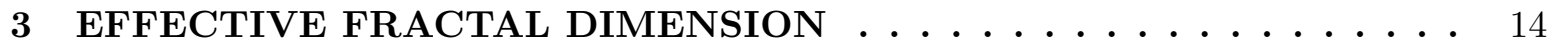

3.1 Hausdorff and Packing Dimensions . . . . . . . . . . . . . . . 14

3.1.1 Gale Characterizations . . . . . . . . . . . . . . . . 16

3.2 Constructive Dimensions . . . . . . . . . . . . . . . . . . . . . 21

3.2.1 Gales Suffice for Constructive Dimension . . . . . . . . . . . . . . . 32

3.2 .2 Entropy Rates . . . . . . . . . . . . . . . . . . . . . 35

3.3 Resource-Bounded Dimensions . . . . . . . . . . . . . . . . . 40 
3.3.1 Log-Loss Unpredictability . . . . . . . . . . . . . . . . . . . 43

3.3.2 Entropy Rates and Kolmogorov Complexity . . . . . . . . . . . . . . . . 48

3.4 Scaled Dimension . . . . . . . . . . . . . . . . . . . . 53

3.4.1 Unpredictability Tools . . . . . . . . . . . . . . . . . . . 57

4 CONSTRUCTIVE DIMENSION AND THE ARITHMETICAL HIER-

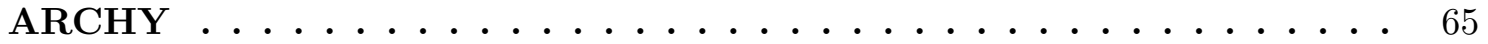

4.1 Effective Borel Hierarchies . . . . . . . . . . . . . . . . . . . . . 65

4.2 Correspondence Principles . . . . . . . . . . . . . . . . . . . . . . 68

4.3 Classification of Dimension Classes . . . . . . . . . . . . . . . . 73

4.3.1 Category Methods ..................... 75

4.3 .2 Wadge Reductions . . . . . . . . . . . . . 76

4.3 .3 Ad Hoc Methods . . . . . . . . . . . . . . . . . . . . . . 83

5 RESOURCE-BOUNDED DIMENSION AND POLYNOMIAL-TIME RE-

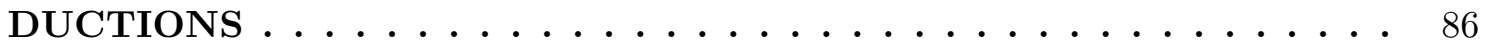

5.1 Small Spans in Scaled Dimension _ . . . . . . . . . . . . . . . . 87

5.1.1 Scaled Non-Bi-Immunity and Compressibility . . . . . . . . . . . . . 89

5.1 .2 Small Span Theorem . . . . . . . . . . . . . . . . . . . . 93

5.1 .3 Lower Spans vs. Degrees in Orders -2 Through $2 \ldots$. . . . . . . . . . 99

$5.1 .4 \leq_{1-\mathrm{tt}^{\mathrm{P}}}^{\mathrm{P}}$-Lower Spans vs. $\leq_{\mathrm{m}}^{\mathrm{p}}$-Lower Spans . . . . . . . . . . . . . . . 104

5.1.5 The Scaled Dimension of $\mathcal{C}_{\mathrm{m}}^{\mathrm{p}}(\mathrm{E})$ in ESPACE . . . . . . . . . . . . 107

5.2 Degrees of Arbitrary Dimensions _ . . . . . . . . . . . . . . . . 110

5.3 Reductions to Nondense Languages . . . . . . . . . . . . . . . . . . 126

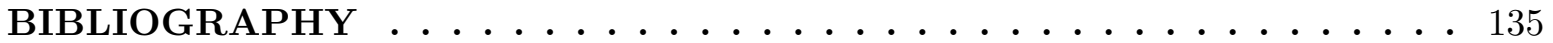




\section{ACKNOWLEDGMENTS}

My foremost thanks go to my advisor Jack Lutz. Jack brought me into research in 1999 while I was still an undergraduate. In the four years since he has provided me with excellent research advice and I have thoroughly enjoyed working with him.

The research presented in this thesis was supported in part by National Science Foundation Grant 9988483. I am grateful to Lance Fortnow for hosting me as a summer intern at NEC Research Institute in Princeton in 2002. In the summer of 2000 I benefited from a research assistantship with Ricky Kendall in the U.S. Department of Energy's Ames Laboratory.

Parts of this dissertation are based on papers co-authored with Krishna Athreya, Jack Lutz, Elvira Mayordomo, and Bas Terwijn. I thank them for collaborating with me. I also thank Pavan Aduri, Cliff Bergman, and Giora Slutzki for working with me.

I thank my mother for encouraging my interest in computer science and mathematics.

Above all, I thank my wife Ruth for her love, understanding, and encouragement. 


\section{INTRODUCTION}

The dimension of an object is a measure of its complexity. Effective fractal dimension makes this idea more precise and useful for theoretical computer science.

Intuitively, in Euclidean space, the dimension of a set is the amount of information that is necessary and sufficient to identify any point in the set. Traditionally, points have dimension 0 as there is nothing to specify, curves have dimension 1 because they can be parametrized by a single real coordinate, and surfaces have dimension 2 since two coordinates describe every point on a surface. However, this intuitive concept of dimension is not completely adequate. For example, a Peano space filling curve includes every point in the unit square, but the unit square should be 2-dimensional.

In 1919, Hausdorff [17] used measure theory to give a mathematically sophisticated notion of dimension that is now most commonly called Hausdorff dimension. This dimension agrees with our intuition for simple sets. Points have Hausdorff dimension 0, simple curves have dimension 1, and simple surfaces have dimension 2. It is possible for complex curves to have dimension larger than 1 and for complex surfaces to have dimension larger than 2. In fact, the Peano space filling curve has Hausdorff dimension 2. The Hausdorff dimension of a set need not be an integer; it can be an arbitrary real number.

Hausdorff dimension has become a powerful tool in fractal geometry [13]. Fractals are sets that typically have simple definitions but still exhibit complex properties. Fractals often have non-integral dimension. One of the simplest examples of a fractal is the Cantor middlethirds set, an uncountable, totally disconnected, measure 0 subset of the unit interval that

has Hausdorff dimension $\frac{\log 2}{\log 3}$. The von Koch curve has Hausdorff dimension $\frac{\log 4}{\log 3}$ and is an example of a fractal in the plane. These dimensions capture the similarity ratios of the Cantor 
set and von Koch curve and can be thought of as a measure of their complexity.

The definition of Hausdorff dimension is sufficiently general to work in any metric space. The Cantor space, consisting of all infinite binary sequences, is a metric space that is of central importance to theoretical computer science. For example, it is standard to identify a decision problem, a subset of the finite binary strings, with the element of Cantor space that is its characteristic sequence. In this way, complexity classes, classes of decision problems, can be viewed as subsets of Cantor space. However, most interesting complexity classes are countable and all countable sets have Hausdorff dimension 0, so Hausdorff dimension is not a useful measure of complexity for studying these classes.

This problem of countability also occurs when Lebesgue measure is used as a notion of size for complexity classes - all interesting classes have measure 0. Lutz [33] overcame this when he introduced resource-bounded measure, an effective version of Lebesgue measure. The basis for this effectivization is Ville's theorem [62] that gives an equivalent way to define the Lebesgue measure 0 sets using martingales, functions which represent a gambler's capital in a fair game. Martingales were introduced by Lévy [27] and Ville [62] and have also been used extensively by Schnorr $[52,53,54]$ and others in the investigation of algorithmic randomness and constructive measure.

Lutz defined the notion of resource-bounded measure 0 by imposing appropriate computability and complexity constraints on the martingales from Ville's theorem. The resource bound is a parameter in the definition; the special cases of computable measure, polynomialspace measure, and polynomial-time measure are of particular interest. These measures define a reasonable and interesting notion of size within the class DEC of decidable problems and also within various countable complexity classes including the class ESPACE of problems decidable in exponential space and the class $\mathrm{E}$ of problems decidable in exponential time. In particular, DEC does not have computable measure 0, ESPACE does not have polynomial-space measure, and E does not have polynomial-time measure 0. On the other hand, subclasses of these classes can have resource-bounded measure 0.

More recently, Lutz $[30,31]$ has also effectivized Hausdorff dimension in much the same 
way. The key is an analogue of Ville's theorem that characterizes Hausdorff dimension using gales, generalizations of martingales where the gambling game is not fair. This yields a new, simpler definition of Hausdorff dimension for Cantor space. Computability and complexity constraints are placed on these gales to define a variety of effective fractal dimensions including constructive dimension and resource-bounded dimension. Constructive dimension is fundamentally related to algorithmic randomness and provides a definition of dimension for individual elements of Cantor space. Resource-bounded dimension is defined using a general resource bound as a parameter (just like resource-bounded measure) and includes computable dimension, polynomial-space dimension, and polynomial-time dimension as special cases. These dimensions have the same relationship with resource-bounded measure as Hausdorff dimension has with Lebesgue measure.

These effective dimensions serve as a useful notion of complexity for theoretical computer science. They have been shown to be closely related to several measures of complexity including Kolmogorov complexity [43, 31, 23], Boolean circuit-size complexity [30, 23], and predictability [15]. Applications have also been given to the study of polynomial-time degrees [2] and approximate optimization [19].

In Chapter 3 we present the theory of effective fractal dimension and make several contributions to it. Chapter 4 investigates relationships between constructive dimension and the arithmetical hierarchy. Classes involving polynomial-time reductions are studied using resource-bounded dimension in Chapter 5. The remainder of this introduction is a summary of the main contributions of this dissertation.

\subsection{Foundations of Effective Fractal Dimension}

In Chapter 3 we define the effective fractal dimensions and present their basic properties. Along the way, several foundational results are proved. We now highlight some of them. 


\subsubsection{Effective Strong Dimension}

In the 1980s, a new concept of fractal dimension, called the packing dimension, was introduced independently by Tricot [61] and Sullivan [59]. Packing dimension shares with Hausdorff dimension the mathematical advantage of being based on a measure. Over the past two decades, despite its greater complexity (requiring an extra optimization over all countable decompositions of a set in its definition), packing dimension has become, next to Hausdorff dimension, the most important notion of fractal dimension, yielding extensive applications [13]. Packing dimension agrees with Hausdorff dimension for sets that are sufficiently regular, but the two dimensions can be different.

After reviewing Lutz's gale characterization of Hausdorff dimension, we prove that packing dimension can also be characterized using gales in Section 3.1.1. Moreover, notwithstanding the greater complexity of packing dimension's definition (and the greater complexity of its behavior on compact sets, as established by Mattila and Mauldin [41]), our gale characterization of packing dimension is an exact dual of - and every bit as simple as - the gale characterization of Hausdorff dimension. (This duality and simplicity are in the statement of our gale characterization; its proof is more involved than its counterpart for Hausdorff dimension.)

Effectivizing our gale characterization of packing dimension produces for each of Lutz's

effective dimensions an effective strong dimension that is its exact dual. Just as the Hausdorff dimension of a set is bounded above by its packing dimension, the effective dimension of a set is bounded above by its effective strong dimension. Moreover, just as in the classical case, the effective dimension coincides with the strong effective dimension for sets that are sufficiently regular.

Throughout Chapter 3 we present the theory of effective strong dimension alongside that of effective dimension. The material on packing dimension and effective strong dimension is joint work with Athreya, Lutz, and Mayordomo and is based on [5]. 


\subsubsection{Gales Suffice for Constructive Dimension}

Lutz [31] originally formulated constructive dimension using supergales, functions which are similar to gales but lack an equality property. (Supergales are to gales as supermartingales are to martingales.) For the classical and resource-bounded dimensions, it is easy to see that gales and supergales give equivalent definitions. For constructive dimension, Lutz used supergales rather than gales because he was able to show that optimal constructive supergales exist. These optimal supergales give constructive dimension some very nice properties. The questions of whether optimal constructive gales exist and whether gales can be used to equivalently define constructive dimension were left open. In Section 3.2.1 we show that certain forms of optimal constructive gales do exist and that gales and supergales are equivalent for defining constructive dimension. This section is based on [21]. The main result is also due independently to Fenner [14].

\subsubsection{Entropy Rates and Kolmogorov Complexity}

For each set $X$ of sequences, Staiger [57] defined a kind of entropy rate that coincides with classical Hausdorff dimension and proved results relating a computable version of this entropy rate to Hausdorff dimension. In Section 3.2.2 we show that a constructive version of Staiger's entropy rate gives an analogous characterization of constructive dimension. We show in Section 3.3.2 that Staiger's computable entropy rate coincides with computable dimension and also that a polynomial-space entropy rate characterizes polynomial-space dimension. The material on entropy rates is based on [18].

There is a natural relationship between entropy rates and Kolmogorov complexity. In Section 3.3.2 we use this to extend Mayordomo's Kolmogorov complexity characterization

of constructive dimension [43] to the computable and polynomial-space dimensions by using resource-bounded Kolmogorov complexity. 


\subsubsection{Log-Loss Unpredictability}

Given a set $X$ of infinite sequences over a finite alphabet, consider the problem of designing a single predictor that performs well on all sequences in $X$. We define the log-loss unpredictability of $X$ as the minimal average log-loss rate that a predictor can achieve on all members of $X$; here the rate is given by a limit inferior. Using a limit superior, the strong log-loss unpredictability of $X$ is defined analogously. In Section 3.3.1 we show that log-loss unpredictability is Hausdorff dimension and that strong log-loss unpredictability is packing dimension. This extends to resource-bounded dimension by imposing resource-bounds on the predictors to define resource-bounded unpredictabilities. This result explains and illuminates the relationships between prediction and Hausdorff dimension that were obtained by Fortnow and Lutz [15], Ryabko [49, 50, 51], and Staiger [57]. This section is based on [20].

To overcome limitations of resource-bounded dimension for investigating certain complex-

ity classes within ESPACE, Hitchcock, Lutz, and Mayordomo [23] introduced a theory of resource-bounded scaled dimension. This is in analogy with the classical theory of generalized dimension (see [47]) that arises naturally from Hausdorff dimension. In Section 3.4 we review scaled dimension and develop some log-loss unpredictability tools for working with it. This section is based on [22].

\subsection{The Arithmetical Hierarchy}

In Chapter 4 we investigate relationships between the arithmetical hierarchy and constructive dimension. We identify the levels of the arithmetical hierarchy in which the Hausdorff and constructive dimensions of a set are guaranteed to be equal. The constructive dimension classes are precisely located in the arithmetical hierarchy.

\subsubsection{Correspondence Principles}

In early lectures on effective dimension [36], Lutz conjectured that there should be a correspondence principle stating that the constructive dimension of every sufficiently simple 
set $X$ coincides with its classical Hausdorff dimension. In Section 4.2 we provide such a principle, along with a new proof of an analogous correspondence principle for computable dimension. Our correspondence principle for constructive dimension says that for every set $X$ that is an arbitrary union of $\Pi_{1}^{0}$-definable sets of sequences, the constructive and Hausdorff dimensions of $X$ are equal. The correspondence principle for computable dimension (which was first proven by Staiger [57]) says that for every $\Sigma_{2}^{0}$-definable set $X$ of sequences, the computable and Hausdorff dimensions of $X$ are equal. We show that these results are optimal in the arithmetical hierarchy. This section is based on [18].

\subsubsection{Complexity of Dimension Classes}

Just as Martin-Löf [40] used constructive measure to define the randomness of individual sequences, Lutz [31] used constructive dimension to define the dimensions of individual sequences. Each element $S$ of Cantor space is assigned a dimension and a strong dimension which are the constructive dimension of $\{S\}$ and the constructive strong dimension of $\{S\}$, respectively.

For any $\alpha \in[0,1]$, let $\mathrm{DIM}^{\alpha}$ be the class of all sequences that have dimension $\alpha$ and let $\mathrm{DIM}_{\mathrm{str}}^{\alpha}$ be the class of all sequences that have strong dimension $\alpha$. We investigate the complexities of these dimension classes in terms of the arithmetical hierarchy of subsets of Cantor space. We show that $\mathrm{DIM}^{0}$ is properly $\Pi_{2}^{0}$, and for all $\Delta_{2}^{0}$-computable $\alpha \in(0,1]$ we show that $\mathrm{DIM}^{\alpha}$ is properly $\Pi_{3}^{0}$. To classify the strong dimension classes, we introduce a more powerful effective Borel hierarchy where a co-enumerable predicate is used rather than a enumerable predicate in the definition of the $\Sigma_{1}^{0}$ level. We show that $\operatorname{DIM}_{\text {str }}^{1}$ is properly in the $\Pi_{2}^{0}$ level of this stronger hierarchy. For all $\Delta_{2}^{0}$-computable $\alpha \in[0,1)$, we show that $\operatorname{DIM}_{\mathrm{str}}^{\alpha}$ is properly in the $\Pi_{3}^{0}$ level of this hierarchy. This section is joint work with Lutz and Terwijn and is based on [24]. 


\subsection{Polynomial-Time Reductions}

In Chapter 5 we use resource-bounded dimension to investigate complexity classes involving polynomial-time reductions.

\subsubsection{Small Spans}

In Section 5.1 we use resource-bounded scaled dimension to strengthen from both ends the contrasting theorems of Juedes and Lutz [25] and Ambos-Spies, Merkle, Reimann, and Stephan [2] regarding spans under polynomial-time reductions.

1. The small span theorem of [25] for $\leq_{\mathrm{m}}^{\mathrm{p}}$-reductions [25] is strengthened from resourcebounded measure to $-3^{\text {rd }}$-order resource-bounded scaled dimension.

2. The result of [2] that $\leq_{\mathrm{m}}^{\mathrm{p}}$-lower spans and -degrees have the same dimension in $\mathrm{E}$ is extended to all orders $-2 \leq i \leq 2$ of scaled dimension in E. This implies that there is no small span theorem in $-2^{\text {nd }}$-order scaled dimension in E.

These results suggest that contrast between the $-2^{\text {nd }}-$ and $-3^{\text {rd }}$-orders of resource-bounded scaled dimension will be useful for studying complexity classes involving polynomial-time reductions. For example, our results imply that the many-one complete degree of NP unconditionally has $-3^{\text {rd }}$-order scaled dimension 0 in $\mathrm{E}$, but that in order -2 the scaled dimension of the complete degree is the same as the scaled dimension of NP. Scaled dimension therefore provides two different types of dimension for studying NP. The NP-complete degree provides all the dimension of NP in order -2 , but in order -3 the NP-complete degree unconditionally has dimension 0 .

We also prove analogous results for scaled dimension in ESPACE. We are able to show that determining the $-1^{\text {st }}$ - or $-2^{\text {nd }}$-order scaled dimension of the class of complete languages for E within ESPACE would fully derandomize BPP or separate P from PSPACE. In contrast, without any hypothesis we show that the complete languages for $\mathrm{E}$ have $-3^{\text {rd }}$-order dimension 0 in ESPACE and $-2^{\text {nd }}$ - and $-1^{\text {st }}$-order dimension 1 in E.

This section is based on [22]. 


\subsubsection{Degrees of Arbitrary Dimensions}

Ambos-Spies, Merkle, Reimann, and Stephan [2] showed that for any $\Delta_{2}^{0}$-computable real number $\alpha$ there is a decision problem $A \in \mathrm{E}$ such that the polynomial-time many-one degree of $A$ has dimension $\alpha$ in E. In Section 5.2 we extend this result to show that for every pair of $\Delta_{2}^{0}$-computable real numbers $0 \leq \alpha \leq \beta \leq 1$ there is a decision problem $A \in \mathrm{E}$ such that the polynomial-time many-one degree of $A$ has dimension $\alpha$ in $\mathrm{E}$ and strong dimension $\beta$ in E. This extension uses our log-loss unpredictability characterization of polynomial-time dimension.

This section is joint work with Athreya, Lutz, and Mayordomo and is based on [5].

\subsubsection{Reductions to Nondense Languages}

Polynomial-time measure has been used by Lutz and Mayordomo [37] and Lutz and Zhao [39] to investigate the size of the class of problems that can be efficiently reduced to languages that are not exponentially dense. In order to use polynomial-time dimension to understand the frequency of inapproximability for the MAX3SAT optimization problem, a result similar to those in [37, 39] (but using weaker reductions) was proved in [19] for polynomial-time dimension. In Section 5.3 we strengthen the result of [19] to more powerful reductions. This gives a new proof of a result due to Watanabe [63] regarding the densities of hard languages for exponential time. 


\section{PRELIMINARIES}

We use the set $\mathbb{Z}$ of integers, the set $\mathbb{Z}^{+}$of (strictly) positive integers, the set $\mathbb{N}$ of natural numbers (i.e., nonnegative integers), the set $\mathbb{Q}$ of rational numbers, the set $\mathbb{R}$ of real numbers, and the set $[0, \infty)$ of nonnegative reals. All logarithms in this thesis are base 2 . We use the slow-growing function $\log ^{*} n=\min \left\{j \in \mathbb{N} \mid t_{j} \geq n\right\}$, where $t_{0}=0$ and $t_{j+1}=2^{t_{j}}$, and Shannon's binary entropy function $\mathcal{H}:[0,1] \rightarrow[0,1]$ defined by

$$
\mathcal{H}(\beta)=\beta \log \frac{1}{\beta}+(1-\beta) \log \frac{1}{1-\beta}
$$

where $0 \log \frac{1}{0}=0$.

A language, or decision problem, is a set $A \subseteq\{0,1\}^{*}$. We usually identify a language $A$ with its characteristic sequence $\chi_{A} \in \mathbf{C}$ defined by $\chi_{A}[n]=$ if $s_{n} \in A$ then 1 else 0 , where $s_{0}=\lambda, s_{1}=0, s_{2}=1, s_{3}=00, \ldots$ is the standard enumeration of $\{0,1\}^{*}$. That is, we usually (but not always) use $A$ to denote both the set $A \subseteq\{0,1\}^{*}$ and the sequence $A=\chi_{A} \in \mathbf{C}$. In the same way, classes of languages are routinely identified with subsets of Cantor space.

A string is a finite, binary string $w \in\{0,1\}^{*}$. We write $|w|$ for the length of a string $w$ and $\lambda$ for the empty string. For $i, j \in\{0, \ldots,|w|-1\}$, we write $w[i . . j]$ for the string consisting of the $i^{\text {th }}$ through the $j^{\text {th }}$ bits of $w$ and $w[i]$ for $w[i . . i]$, the $i^{\text {th }}$ bit of $w$. Note that the $0^{\text {th }}$ bit $w[0]$ is the leftmost bit of $w$ and that $w[i . . j]=\lambda$ if $i>j$. We also define $w\lceil i=w[0 . . i-1]$ as the first $i$ bits of $w$. A sequence is an infinite, binary sequence. If $S$ is a sequence and $i, j \in \mathbb{N}$, then the notations $S[i . . j], S[i], S \uparrow i$ are defined exactly as for strings. We work in the Cantor space $\mathbf{C}$ consisting of all sequences. A string $w \in\{0,1\}^{*}$ is a prefix of a sequence $S \in \mathbf{C}$, and we write $w \sqsubseteq S$, if $S \uparrow|w|=w$. The cylinder generated by a string $w \in\{0,1\}^{*}$ is 
$\mathbf{C}_{w}=\{S \in \mathbf{C} \mid w \sqsubseteq S\}$. Note that $\mathbf{C}_{\lambda}=\mathbf{C}$.

Given a set $A \subseteq\{0,1\}^{*}$ and $n \in \mathbb{N}$, we use the abbreviations $A_{=n}=A \cap\{0,1\}^{n}$ and $A_{\leq n}=A \cap\{0,1\}^{\leq n}$. A prefix set is a set $A \subseteq\{0,1\}^{*}$ such that no element of $A$ is a prefix of another element of $A$.

For each $i \in \mathbb{N}$ we define a class $G_{i}$ of functions from $\mathbb{N}$ into $\mathbb{N}$ as follows [33].

$$
\begin{aligned}
G_{0} & =\left\{f \mid(\exists k)\left(\forall^{\infty} n\right) f(n) \leq k n\right\} \\
G_{i+1} & =2^{G_{i}(\log n)}=\left\{f \mid\left(\exists g \in G_{i}\right)\left(\forall^{\infty} n\right) f(n) \leq 2^{g(\log n)}\right\}
\end{aligned}
$$

We also define the functions $\hat{g}_{i} \in G_{i}$ by $\hat{g}_{0}(n)=2 n, \hat{g}_{i+1}(n)=2^{\hat{g}_{i}(\log n)}$. We regard the functions in these classes as growth rates. In particular, $G_{0}$ contains the linearly bounded growth rates and $G_{1}$ contains the polynomially bounded growth rates. It is easy to show that each $G_{i}$ is closed under composition, that each $f \in G_{i}$ is $o\left(\hat{g}_{i+1}\right)$, and that each $\hat{g}_{i}$ is $o\left(2^{n}\right)$. Thus $G_{i}$ contains superpolynomial growth rates for all $i>1$, but all growth rates in the $G_{i}$-hierarchy are subexponential.

Let CE be the class of computably enumerable languages. Within the class DEC of all decidable languages, we are interested in the exponential complexity classes $\mathrm{E}_{i}=\operatorname{DTIME}\left(2^{G_{i-1}}\right)$ and $\mathrm{E}_{i} \mathrm{SPACE}=\operatorname{DSPACE}\left(2^{G_{i-1}}\right)$ for $i \geq 1$. The much-studied classes $\mathrm{E}=\mathrm{E}_{1}=\operatorname{DTIME}\left(2^{\text {linear }}\right)$, $\mathrm{EXP}=\mathrm{E}_{2}=\operatorname{DTIME}\left(2^{\text {polynomial }}\right), \operatorname{ESPACE}=\mathrm{E}_{1} \mathrm{SPACE}=\operatorname{DSPACE}\left(2^{\text {linear }}\right)$, and $\operatorname{EXPSPACE}=$ $\mathrm{E}_{2} \mathrm{SPACE}=\mathrm{DSPACE}\left(2^{\text {polynomial }}\right)$ are of particular interest.

We use the following classes of functions.

$$
\begin{aligned}
\text { all } & =\left\{f \mid f:\{0,1\}^{*} \rightarrow\{0,1\}^{*}\right\} \\
\text { comp } & =\{f \in \text { all } \mid f \text { is computable }\} \\
\mathrm{p}_{i} & =\left\{f \in \text { all } \mid f \text { is computable in } G_{i} \text { time }\right\}(i \geq 1) \\
\mathrm{p}_{i} \text { space } & =\left\{f \in \text { all } \mid f \text { is computable in } G_{i} \text { space }\right\}(i \geq 1)
\end{aligned}
$$

(The length of the output is included as part of the space used in computing $f$.) We write p 
for $\mathrm{p}_{1}$ and pspace for $\mathrm{p}_{1}$ space.

A constructor is a function $\delta:\{0,1\}^{*} \rightarrow\{0,1\}^{*}$ that satisfies $x_{\neq}^{\sqsubset} \delta(x)$ for all $x$. The result of a constructor $\delta$ (i.e., the language constructed by $\delta$ ) is the unique language $R(\delta)$ such that $\delta^{n}(\lambda) \sqsubseteq R(\delta)$ for all $n \in \mathbb{N}$. Intuitively, $\delta$ constructs $R(\delta)$ by starting with $\lambda$ and then iteratively generating successively longer prefixes of $R(\delta)$. We write $R(\Delta)$ for the set of languages $R(\delta)$ such that $\delta$ is a constructor in $\Delta$. The following facts are the reason for our interest in the above-defined classes of functions [33].

$$
\begin{aligned}
& R(\text { all })=\mathbf{C} . \\
& R(\text { comp })=\text { DEC } .
\end{aligned}
$$

For $i \geq 1, R\left(\mathrm{p}_{i}\right)=\mathrm{E}_{i}$.

For $i \geq 1, R\left(\mathrm{p}_{i}\right.$ space $)=\mathrm{E}_{i} \mathrm{SPACE}$.

If $D$ is a discrete domain (such as $\mathbb{N},\{0,1\}^{*}, \mathbb{N} \times\{0,1\}^{*}$, etc.), then a function $f: D \longrightarrow$ $[0, \infty)$ is $\Delta$-computable if there is a function $\hat{f}: \mathbb{N} \times D \longrightarrow \mathbb{Q} \cap[0, \infty)$ such that $|\hat{f}(r, x)-f(x)| \leq$ $2^{-r}$ for all $r \in \mathbb{N}$ and $x \in D$ and $\hat{f} \in \Delta$ (with $r$ coded in unary and the output coded in binary). We say that $f$ is exactly $\Delta$-computable if $f: D \longrightarrow \mathbb{Q} \cap[0, \infty)$ and $f \in \Delta$.

Let resource $\in\{$ time, space $\}$ and let $t(n)$ be a resource bound. Let $l \in \mathbb{N}$. A function $f: \mathbb{N}^{l} \times\{0,1\}^{*} \rightarrow[0, \infty) \cap \mathbb{Q}$ is $t(n)$-resource exactly computable if there is a Turing machine that computes $f\left(k_{1}, \ldots, k_{l}, w\right)$ using at most $t\left(k_{1}+\cdots+k_{l}+|w|\right)$ resource for all $\left(k_{1}, \ldots, k_{l}, w\right) \in$ $\mathbb{N}^{l} \times\{0,1\}^{*}$. Let $g: \mathbb{N}^{l} \times\{0,1\}^{*} \rightarrow[0, \infty)$ be a real-valued function. An approximation of $g$ is a function $\hat{g}: \mathbb{N}^{l+1} \times\{0,1\}^{*} \rightarrow[0, \infty)$ such that

$$
|g(x)-\hat{g}(r, x)| \leq 2^{-r}
$$

for all $x \in \mathbb{N}^{l} \times\{0,1\}^{*}$ and $r \in \mathbb{N}$. We say that $g$ is $t(n)$-resource computable if there is an exactly $t(n)$-resource computable approximation $\hat{g}$ of $g$. A family of functions $\left(f_{i}\right.$ : $\mathbb{N}^{l} \times\{0,1\}^{*} \rightarrow[0, \infty) \mid i \in \mathbb{N}$ ) is uniformly $t(n)$-resource (exactly) computable if the function $f(i, x)=f_{i}(x)$ is $t(n)$-resource (exactly) computable.

We say that $f$ is lower semicomputable if there is a computable function $\hat{f}: D \times \mathbb{N} \rightarrow \mathbb{Q}$ 
such that

(a) for all $(x, t) \in D \times \mathbb{N}, \hat{f}(x, t) \leq \hat{f}(x, t+1)<f(x)$, and

(b) for all $x \in D, \lim _{t \rightarrow \infty} \hat{f}(x, t)=f(x)$.

Finally, we say that $f$ is $\Delta_{2}^{0}$-computable if $f$ is computable (i.e., comp-computable) relative to the halting oracle.

A real number $\alpha \in[0, \infty)$ is computable (respectively, $\Delta_{2}^{0}$-computable) if the function $f:\{0\} \rightarrow[0, \infty)$ defined by $f(0)=\alpha$ is computable (respectively, $\Delta_{2}^{0}$-computable). 


\section{EFFECTIVE FRACTAL DIMENSION}

This chapter introduces effective fractal dimension. First we recall the classical Hausdorff and packing dimensions and present their gale characterizations that form the foundation for the constructive and resource-bounded dimensions and strong dimensions.

\subsection{Hausdorff and Packing Dimensions}

We begin this section by reviewing the classical definitions of some fractal dimensions and the relationships among them. Since we are primarily interested in binary sequences and (equivalently) decision problems, we focus on fractal dimension in the Cantor space $\mathbf{C}$.

For each $k \in \mathbb{N}$, we let $\mathcal{A}_{k}$ be the collection of all prefix sets $A$ such that $A_{<k}=\emptyset$. For each $X \subseteq \mathbf{C}$, we then define the families

$$
\begin{aligned}
& \mathcal{A}_{k}(X)=\left\{A \in \mathcal{A}_{k} \mid X \subseteq \bigcup_{w \in A} \mathbf{C}_{w}\right\}, \\
& \mathcal{B}_{k}(X)=\left\{A \in \mathcal{A}_{k} \mid(\forall w \in A) \mathbf{C}_{w} \cap X \neq \emptyset\right\} .
\end{aligned}
$$

If $A \in \mathcal{A}_{k}(X)$, then we say that the prefix set $A$ covers the set $X$. If $A \in \mathcal{B}_{k}(X)$, then we call the prefix set $A$ a packing of $X$. For $X \in \mathbf{C}, s \in[0, \infty)$, and $k \in \mathbb{N}$, we then define

$$
\begin{aligned}
& H_{k}^{s}(X)=\inf _{A \in \mathcal{A}_{k}(X)} \sum_{w \in A} 2^{-s|w|} \\
& P_{k}^{s}(X)=\sup _{A \in \mathcal{B}_{k}(X)} \sum_{w \in A} 2^{-s|w|} .
\end{aligned}
$$


Since $H_{k}^{s}(X)$ and $P_{k}^{s}(X)$ are monotone in $k$, the limits

$$
\begin{aligned}
& H^{s}(X)=\lim _{k \rightarrow \infty} H_{k}^{s}(X), \\
& P_{\infty}^{s}(X)=\lim _{k \rightarrow \infty} P_{k}^{s}(X)
\end{aligned}
$$

exist, though they may be infinite. We then define

$$
P^{s}(X)=\inf \left\{\sum_{i=0}^{\infty} P_{\infty}^{s}\left(X_{i}\right) \mid X \subseteq \bigcup_{i=0}^{\infty} X_{i}\right\}
$$

The set functions $H^{s}$ and $P^{s}$ have the technical properties of an outer measure [13], and the (possibly infinite) quantities $H^{s}(X)$ and $P^{s}(X)$ are thus known as the $s$-dimensional Hausdorff (outer) cylinder measure of $X$ and the s-dimensional packing (outer) cylinder measure of $X$, respectively. The set function $P_{\infty}^{s}$ is not an outer measure; this is the reason for the extra optimization (3.1) in the definition of the packing measure.

Definition. Let $X \subseteq \mathbf{C}$.

1. The Hausdorff dimension of $X$ is $\operatorname{dim}_{H}(X)=\inf \left\{s \in[0, \infty) \mid H^{s}(X)=0\right\}$.

2. The packing dimension of $X$ is $\operatorname{dim}_{P}(X)=\inf \left\{s \in[0, \infty) \mid P^{s}(X)=0\right\}$.

There is a well-known characterization of packing dimension as a modified box dimension. For each $X \subseteq \mathbf{C}$ and $n \in \mathbb{N}$, let

$$
N_{n}(X)=\left|\left\{w \in\{0,1\}^{n} \mid(\exists S \in X) w \sqsubseteq S\right\}\right|
$$

Then the upper box dimension of $X$ is

$$
\overline{\operatorname{dim}}_{\mathrm{B}}(X)=\limsup _{n \rightarrow \infty} \frac{\log N_{n}(X)}{n} .
$$

The lower box dimension $\underline{\operatorname{dim}}_{\mathrm{B}}(X)$, which we do not use here, is obtained by using a limit inferior in place of the limit superior in (3.2). When $\underline{\operatorname{dim}}_{\mathrm{B}}(X)=\overline{\operatorname{dim}}_{\mathrm{B}}(X)$, this quantity, 
written $\operatorname{dim}_{\mathrm{B}}(X)$, is called the box dimension of $X$.

Box dimensions are over 60 years old, have been re-invented many times, and have been named many things, including Minkowski dimension, Kolmogorov entropy, Kolmogorov dimension, topological entropy, metric dimension, logarithmic density, and information dimension. Box dimensions are often used in practical applications of fractal geometry because they are easy to estimate, but they are not well-behaved mathematically. The modified upper box dimension

$$
\overline{\operatorname{dim}}_{\mathrm{MB}}(X)=\inf \left\{\sup _{i} \overline{\operatorname{dim}}_{\mathrm{B}}\left(X_{i}\right) \mid X \subseteq \bigcup_{i=0}^{\infty} X_{i}\right\}
$$

is much better behaved. (Note that (3.3), like (3.1), is an optimization over all countable decompositions of $X$.) In fact, the following relations are well-known [13].

Theorem 3.1. For all $X \subseteq \mathbf{C}, 0 \leq \operatorname{dim}_{\mathrm{H}}(X) \leq \overline{\operatorname{dim}}_{\mathrm{MB}}(X)=\operatorname{dim}_{\mathrm{P}}(X) \leq \overline{\operatorname{dim}}_{\mathrm{B}}(X) \leq 1$.

The above dimensions are monotone, i.e., $X \subseteq Y$ implies $\operatorname{dim}(X) \leq \operatorname{dim}(Y)$, and stable, i.e., $\operatorname{dim}(X \cup Y)=\max \{\operatorname{dim}(X), \operatorname{dim}(Y)\}$. The Hausdorff and packing dimensions are also countably stable, i.e., $\operatorname{dim}\left(\cup_{i=0}^{\infty} X_{i}\right)=\sup \left\{\operatorname{dim}\left(X_{i}\right) \mid i \in \mathbb{N}\right\}$.

\subsubsection{Gale Characterizations}

In this subsection we review the gale characterization of Hausdorff dimension and prove the dual gale characterization of packing dimension.

Definition. Let $s \in[0, \infty)$.

1. An s-supergale is a function $d:\{0,1\}^{*} \longrightarrow[0, \infty)$ that satisfies the condition

$$
d(w) \geq 2^{-s}[d(w 0)+d(w 1)]
$$

for all $w \in\{0,1\}^{*}$.

2. An s-gale is an s-supergale that satisfies (3.4) with equality for all $w \in\{0,1\}^{*}$.

3. A supermartingale is a 1-supergale. 
4. A martingale is a 1-gale.

Intuitively, we regard a supergale $d$ as a strategy for betting on the successive bits of a sequence $S \in \mathbf{C}$. More specifically, $d(w)$ is the amount of capital that $d$ has after betting on the prefix $w$ of $S$. If $s=1$, then the right-hand side of (3.4) is the conditional expectation of $d(w b)$ given that $w$ has occurred (when $b$ is a uniformly distributed binary random variable). Thus a martingale models a gambler's capital when the payoffs are fair. (The expected capital after the bet is the actual capital before the bet.) In the case of an $s$-gale, if $s<1$, the payoffs are less than fair; if $s>1$, the payoffs are more than fair.

We use the following known generalization of the Kraft inequality.

Lemma 3.2. (Lutz [30]) Let $s \in[0, \infty)$. If $d$ is an s-supergale and $B \subseteq\{0,1\}^{*}$ is a prefix set, then for all $w \in\{0,1\}^{*}, \sum_{u \in B} 2^{-s|u|} d(w u) \leq d(w)$.

We now define two criteria for the success of a supergale.

Definition. Let $d$ be an s-supergale, where $s \in[0, \infty)$.

1. We say that $d$ succeeds on a sequence $S \in \mathbf{C}$ if

$$
\limsup _{n \rightarrow \infty} d(S \uparrow n)=\infty
$$

The success set of $d$ is $S^{\infty}[d]=\{S \in \mathbf{C} \mid d$ succeeds on $S\}$.

2. We say that $d$ succeeds strongly on a sequence $S \in \mathbf{C}$ if

$$
\liminf _{n \rightarrow \infty} d(S \uparrow n)=\infty
$$

The strong success set of $d$ is $S_{\mathrm{str}}^{\infty}[d]=\{S \in \mathbf{C} \mid d$ succeeds strongly on $S\}$.

We have written conditions (3.5) and (3.6) in a fashion that emphasizes their duality. Condition (3.5) simply says that the set of values $d(S \uparrow n)$ is unbounded, while condition (3.6) says that $d(S\lceil n) \rightarrow \infty$ as $n \rightarrow \infty$. 
The following characterization of Lebesgue measure using martingales is foundational to resource-bounded measure.

Theorem 3.3. (Ville [62]) Let $X \subseteq \mathbf{C}$. The following conditions are equivalent.

1. $X$ has Lebesgue measure 0 in $\mathbf{C}$.

2. There is a martingale $d$ with $X \subseteq S^{\infty}[d]$.

3. There is a martingale $d$ with $X \subseteq S_{\mathrm{str}}^{\infty}[d]$.

Ville [62] proved the equivalence of the first two conditions. The equivalence of the second two conditions is well known and easy to prove. We note that replacing "martingale" by "supermartingale" in the theorem yields further equivalent conditions.

The Hausdorff and packing dimensions can be similarly characterized using gales and supergales. For this, we define the following sets of real numbers.

Notation. Let $X \subseteq \mathbf{C}$.

1. $\mathcal{G}(X)$ is the set of all $s \in[0, \infty)$ for which there exists an $s$-gale $d$ such that $X \subseteq S^{\infty}[d]$.

2. $\mathcal{G}^{\operatorname{str}}(X)$ is the set of all $s \in[0, \infty)$ for which there exists an $s$-gale $d$ such that $X \subseteq S_{\text {str }}^{\infty}[d]$.

3. $\widehat{\mathcal{G}}(X)$ is the set of all $s \in[0, \infty)$ for which there exists an $s$-supergale $d$ such that $X \subseteq S^{\infty}[d]$.

4. $\widehat{\mathcal{G}}^{\text {str }}(X)$ is the set of all $s \in[0, \infty)$ for which there exists an $s$-supergale $d$ such that $X \subseteq S_{\mathrm{str}}^{\infty}[d]$.

Note that $s^{\prime} \geq s \in \mathcal{G}(X)$ implies that $s^{\prime} \in \mathcal{G}(X)$, and similarly for the classes $\mathcal{G}^{\operatorname{str}}(X)$, $\widehat{\mathcal{G}}(X)$, and $\widehat{\mathcal{G}}^{\text {str }}(X)$. The following fact is also clear.

Observation 3.4. For all $X \subseteq \mathbf{C}, \mathcal{G}(X)=\widehat{\mathcal{G}}(X)$ and $\mathcal{G}^{\operatorname{str}}(X)=\widehat{\mathcal{G}}^{\operatorname{str}}(X)$.

For Hausdorff dimension, Lutz proved the following fundamental theorem. 
Theorem 3.5. (Gale Characterization of Hausdorff Dimension - Lutz [30]) For all $X \subseteq \mathbf{C}$,

$$
\operatorname{dim}_{\mathrm{H}}(X)=\inf \mathcal{G}(X)
$$

In analogy with Ville's martingale characterization of Lebesgue measure in Theorem 3.3, Lutz proved the gale characterization of Hausdorff dimension by showing that for all $X \subseteq \mathbf{C}$, $H^{s}(X)=0$ if and only if there is an $s$-gale $d$ with $X \subseteq S^{\infty}[d]$.

The following theorem is a dual of Theorem 3.5 for packing dimension.

Theorem 3.6. (Gale Characterization of Packing Dimension) For all $X \subseteq \mathbf{C}$,

$$
\operatorname{dim}_{P}(X)=\inf \mathcal{G}^{\operatorname{str}}(X)
$$

We will use the following lemma to prove Theorem 3.6.

Lemma 3.7. For each family of sets $\left\{X_{k} \subseteq \mathbf{C} \mid k \in \mathbb{N}\right\}$, inf $\mathcal{G}^{\operatorname{str}}\left(\bigcup_{k} X_{k}\right)=\sup _{k} \inf \mathcal{G}^{\operatorname{str}}\left(X_{k}\right)$. Proof. The inequality inf $\mathcal{G}^{\operatorname{str}}\left(\bigcup_{k} X_{k}\right) \geq \sup _{k} \inf \mathcal{G}^{\operatorname{str}}\left(X_{k}\right)$ holds trivially.

To prove that inf $\mathcal{G}^{\operatorname{str}}\left(\bigcup_{k} X_{k}\right) \leq \sup _{k} \inf \mathcal{G}^{\operatorname{str}}\left(X_{k}\right)$, let $s>\sup _{k} \inf \mathcal{G}^{\operatorname{str}}\left(X_{k}\right)$. Then for each $k \in \mathbb{N}$ there is an $s$-gale $d_{k}$ such that $X_{k} \subseteq S_{\text {str }}^{\infty}\left[d_{k}\right]$. We define an $s$-gale $d$ by

$$
d(w)=\sum_{k \in \mathbb{N}} \frac{2^{-k}}{d_{k}(\lambda)} \cdot d_{k}(w)
$$

for all $w \in\{0,1\}^{*}$. Then for each $k$, for any $S \in X_{k}$, we have

$$
d(S \uparrow n) \geq \frac{2^{-k}}{d_{k}(\lambda)} \cdot d_{k}(S \uparrow n)
$$

for all $n$, so $S \in S_{\text {str }}^{\infty}[d]$. Therefore $\bigcup_{k} X_{k} \subseteq S_{\text {str }}^{\infty}[d]$ and the lemma follows.

Proof of Theorem 3.6. Let $X \subseteq$ C. By Theorem 3.1, it suffices to show that $\overline{\operatorname{dim}}_{\mathrm{MB}}(X)=$ $\inf \mathcal{G}^{\operatorname{str}}(X)$.

To see that $\overline{\operatorname{dim}}_{\mathrm{MB}}(X) \leq \inf \mathcal{G}^{\operatorname{str}}(X)$, let $s>\inf \mathcal{G}^{\operatorname{str}}(X)$. It suffices to show that $\overline{\operatorname{dim}}_{\mathrm{MB}}(X) \leq s$. 
By our choice of $s$, there is an $s$-gale $d$ such that $X \subseteq S_{\mathrm{str}}^{\infty}[d]$. For each $n \in \mathbb{N}$, let

$$
B_{n}=\left\{w \in\{0,1\}^{n} \mid d(w)>d(\lambda)\right\}
$$

and

$$
Y_{n}=\left\{S \in \mathbf{C} \mid S \uparrow n \in B_{n}\right\}
$$

For each $i \in \mathbb{N}$, let

$$
X_{i}=\bigcap_{n=i}^{\infty} Y_{n}
$$

and note that

$$
X \subseteq \bigcup_{i=0}^{\infty} X_{i}
$$

For all $n \geq i \in \mathbb{N}$, we have $X_{i} \subseteq Y_{n}$, whence the generalized Kraft inequality (Lemma 3.2) tells us that

$$
N_{n}\left(X_{i}\right) \leq N_{n}\left(Y_{n}\right)=\left|B_{n}\right|<2^{s n}
$$

It follows that, for all $i \in \mathbb{N}$,

$$
\overline{\operatorname{dim}}_{\mathrm{B}}\left(X_{i}\right)=\limsup _{n \rightarrow \infty} \frac{\log N_{n}\left(X_{i}\right)}{n} \leq s
$$

whence by (3.7),

$$
\overline{\operatorname{dim}}_{\mathrm{MB}}(X) \leq \sup _{i \in \mathbb{N}} \overline{\operatorname{dim}}_{\mathrm{B}}\left(X_{i}\right) \leq s
$$

To see that $\inf \mathcal{G}^{\operatorname{str}}(X) \leq \overline{\operatorname{dim}}_{\mathrm{MB}}(X)$, let $s>s^{\prime}>s^{\prime \prime}>\overline{\operatorname{dim}}_{\mathrm{MB}}(X)$. It suffices to show that $\inf \mathcal{G}^{\operatorname{str}}(X) \leq s$. Since $s^{\prime \prime}>\overline{\operatorname{dim}}_{\mathrm{MB}}(X)$, there exist sets $X_{0}, X_{1}, \ldots \subseteq \mathbf{C}$ such that $X=\bigcup_{i=0}^{\infty} X_{i}$ and $\overline{\operatorname{dim}}_{\mathrm{B}}\left(X_{i}\right)<s^{\prime \prime}$ for all $i \in \mathbb{N}$. By Lemma 3.7, it suffices to show that $s \in \mathcal{G}^{\operatorname{str}}\left(X_{i}\right)$ for all $i \in \mathbb{N}$.

Fix $i \in \mathbb{N}$. Since $\overline{\operatorname{dim}}_{\mathrm{B}}\left(X_{i}\right)<s^{\prime \prime}$, there exists $n_{0} \in \mathbb{N}$ such that, for all $n \geq n_{0}, \frac{\log N_{n}\left(X_{i}\right)}{n}<$ $s^{\prime \prime}$, i.e., $N_{n}\left(X_{i}\right)<2^{s^{\prime \prime} n}$. For each $n \geq n_{0}$, let

$$
A_{n}=\left\{S\left\lceil n \mid S \in X_{i}\right\}\right.
$$


(noting that $\left|A_{n}\right|=N_{n}\left(X_{i}\right)$ ), and define $d_{n}:\{0,1\}^{*} \rightarrow[0, \infty)$ by

$$
d_{n}(w)= \begin{cases}2^{\left(s-s^{\prime}\right)|w|} \sum_{\substack{u \\ w u \in A_{n}}} 2^{-s^{\prime}|u|} & \text { if }|w| \leq n \\ 2^{(s-1)(|w|-n)} d_{n}(w\lceil n) & \text { if }|w|>n .\end{cases}
$$

It is routine to verify that $d_{n}$ is an $s$-gale for each $n \geq n_{0}$. Note also that $d_{n}(w)=2^{\left(s-s^{\prime}\right) n}$ for all $n \geq n_{0}$ and $w \in A_{n}$. Let $d=\sum_{n=n_{0}}^{\infty} d_{n}$. Then

$$
\begin{aligned}
d(\lambda) & =\sum_{n=n_{0}}^{\infty} d_{n}(\lambda)=\sum_{n=n_{0}}^{\infty}\left|A_{n}\right| 2^{-s^{\prime} n}=\sum_{n=n_{0}}^{\infty} N_{n}\left(X_{i}\right) 2^{-s^{\prime} n} \\
& <\sum_{n=n_{0}}^{\infty} 2^{\left(s^{\prime \prime}-s^{\prime}\right) n}<\infty
\end{aligned}
$$

so $d$ is an $s$-gale by linearity. Let $S \in X_{i}$. Then, for all $n \geq n_{0}, S\left\lceil n \in A_{n}\right.$, so

$$
d\left(S\lceil n) \geq d_{n}\left(S\lceil n) \geq 2^{\left(s-s^{\prime}\right) n} .\right.\right.
$$

Thus $S \in S_{\text {str }}^{\infty}[d]$. This shows that $X_{i} \subseteq S_{\text {str }}^{\infty}[d]$, whence $s \in \mathcal{G}^{\operatorname{str}}\left(X_{i}\right)$.

By Observation 3.4, we could equivalently use $\widehat{\mathcal{G}}(X)$ and $\widehat{\mathcal{G}}^{\text {str }}(X)$ in Theorems 3.5 and 3.6, respectively.

\subsection{Constructive Dimensions}

In this section we present the constructive dimensions, the most fundamental of the effective dimensions. Constructive dimension was introduced by Lutz [31] as an effectivization of Hausdorff dimension by using constructive supergales. Packing dimension is effectivized in the same way to give constructive strong dimension.

Definition. An s-supergale $d$ is constructive if it is lower semicomputable.

Notation. Let $X \subseteq \mathbf{C}$. 
1. $\mathcal{G}_{\text {constr }}(X)$ is the set of all $s \in[0, \infty)$ for which there exists a constructive $s$-gale $d$ such that $X \subseteq S^{\infty}[d]$.

2. $\mathcal{G}_{\text {constr }}^{\text {str }}(X)$ is the set of all $s \in[0, \infty)$ for which there exists a constructive $s$-gale $d$ such that $X \subseteq S_{\text {str }}^{\infty}[d]$.

3. $\widehat{\mathcal{G}}_{\text {constr }}(X)$ is the set of all $s \in[0, \infty)$ for which there exists a constructive $s$-supergale $d$ such that $X \subseteq S^{\infty}[d]$.

4. $\widehat{\mathcal{G}}_{\text {constr }}^{\text {str }}(X)$ is the set of all $s \in[0, \infty)$ for which there exists a constructive $s$-supergale $d$ such that $X \subseteq S_{\mathrm{str}}^{\infty}[d]$.

Constructive dimension [31] and constructive strong dimension are defined as follows. This definition is motivated by Theorems 3.5 and 3.6. Intuitively, constructive dimension is a constructive version of Hausdorff dimension and constructive strong dimension is a constructive version of packing dimension.

Definition. Let $X \subseteq \mathbf{C}$.

1. The constructive dimension of $X$ is

$$
\operatorname{cdim}(X)=\inf \widehat{\mathcal{G}}_{\text {constr }}(X) .
$$

2. The constructive strong dimension of $X$ is

$$
\operatorname{cDim}(X)=\inf \widehat{\mathcal{G}}_{\text {constr }}^{\text {str }}(X) .
$$

The constructive dimensions have the following relationship with each other and the Hausdorff and packing dimensions.

Observation 3.8. For any $X \subseteq \mathbf{C}$,

$$
\begin{aligned}
0 & \leq \operatorname{dim}_{\mathrm{H}}(X) \leq \operatorname{cdim}(X) \\
& \left.\leq \operatorname{dim}_{\mathrm{P}}(X) \leq \mathrm{I}\right)
\end{aligned}
$$


It will be very useful to consider the dimensions of individual sequences.

Definition. Let $S \in \mathbf{C}$.

1. The dimension of $S$ is

$$
\operatorname{dim}(S)=\operatorname{cdim}(\{S\})
$$

2. The strong dimension of $S$ is

$$
\operatorname{Dim}(S)=\operatorname{cDim}(\{S\})
$$

Martin-Löf [40] used constructive null sets (Martin-Löf tests) to give a definition of individual randomness for sequences that is now commonly called Martin-Löf randomness. This randomness notion is robust and has many equivalent definitions. In particular, Schnorr $[52,53]$ showed that Martin-Löf randomness can be equivalently defined using constructive martingales and supermartingales. Let RAND be the class of all Martin-Löf random sequences.

Theorem 3.9. (Schnorr $[52,53])$ Let $S \in \mathbf{C}$. The following conditions are equivalent.

1. $S \notin \mathrm{RAND}$.

2. There is a constructive martingale $d$ with $S \in S^{\infty}[d]$.

3. There is a constructive supermartingale $d$ with $S \in S^{\infty}[d]$.

The dimension of individual sequences refines Martin-Löf nonrandomness.

Proposition 3.10. (Lutz [31]) Let $S \in \mathbf{C}$. If $\operatorname{dim}(S)<1$, then $S \notin \mathrm{RAND}$.

Proof. Assume that $\operatorname{dim}(S)<1$ and let $d$ be a constructive $s$-supergale with $S \in S^{\infty}[d]$ and $s<1$. Since $d$ is a supermartingale, $S \notin$ RAND by Theorem 3.9.

Schnorr showed that there are optimal constructive supermartingales and martingales. We will use the following notion of optimality for constructive supergales. 
Definition. Let $d^{*}$ be a supergale and let $\mathcal{D}$ be a class of supergales. We say that $d^{*}$ is multiplicatively optimal for $\mathcal{D}$ if for each $d \in \mathcal{D}$ there is an $\alpha>0$ such that $d^{*}(w) \geq \alpha d(w)$ for all $w \in\{0,1\}^{*}$.

Lutz used Levin's universal constructive semimeasure [64] to show that there exist multiplicatively optimal supergales.

Theorem 3.11. (Lutz [31]) For any computable $s \in[0, \infty)$ there is a constructive s-supergale $\mathbf{d}^{(s)}$ that is multiplicatively optimal for the class of constructive s-supergales.

Proof. A semimeasure is a function $\nu:\{0,1\}^{*} \rightarrow[0, \infty)$ satisfying $\nu(w 0)+\nu(w 1) \leq \nu(w)$ (i.e., a 0-gale). Levin [64] proved that there is a constructive semimeasure $\mathbf{M}$ that is multiplicatively optimal for the class of all constructive semimeasures. The $s$-gale we want is defined by

$$
\mathbf{d}^{(s)}(w)=2^{s|w|} \mathbf{M}(w)
$$

for all $w \in\{0,1\}^{*}$.

Corollary 3.12. Let $X \subseteq$ C. Let $s>\operatorname{cdim}(X)$ and $t>\operatorname{cDim}(X)$ be computable. Then $X \subseteq S^{\infty}\left[\mathbf{d}^{(s)}\right]$ and $X \subseteq S_{\text {str }}^{\infty}\left[\mathbf{d}^{(t)}\right]$.

Proof. Since $s>\operatorname{cdim}(X)$, for some $s^{\prime}<s$ there is a constructive $s^{\prime}$-supergale $d_{s^{\prime}}$ such that $X \subseteq S^{\infty}\left[d_{s^{\prime}}\right]$. Since $s^{\prime}<s, d_{s^{\prime}}$ is also a constructive $s$-supergale, so $S^{\infty}\left[d_{s^{\prime}}\right] \subseteq S^{\infty}\left[\mathbf{d}^{(s)}\right]$ by Theorem 3.11. The proof that $X \subseteq S_{\mathrm{str}}^{\infty}\left[\mathbf{d}^{(t)}\right]$ is analogous.

The following is a cornerstone of constructive dimension theory.

Theorem 3.13. (Lutz [31]) For any $X \subseteq \mathbf{C}$,

$$
\operatorname{cdim}(X)=\sup _{S \in X} \operatorname{dim}(S)
$$

and

$$
\mathrm{cDim}(X)=\sup _{S \in X} \operatorname{Dim}(S)
$$


Proof. We prove the first equality; the proof for strong dimension is analogous. Let

$$
r=\sup _{S \in X} \operatorname{dim}(S)
$$

The inequality $\operatorname{cdim}(X) \geq r$ is clear by monotonicity. For the other inequality, let $t>$ $r$ be computable. For each $S \in X$, we have $\operatorname{dim}(S)<t$, so $S \in S^{\infty}\left[\mathbf{d}^{(t)}\right]$ by Corollary 3.12. Therefore $X \subseteq S^{\infty}\left[\mathbf{d}^{(t)}\right]$ and $\operatorname{cdim}(X) \leq t$. As $t>r$ is an arbitrary computable real, $\operatorname{cdim}(X) \leq r$ follows.

Mayordomo showed that constructive dimension has a fundamental relationship with Kolmogorov complexity.

Theorem 3.14. (Mayordomo [43]) For any $S \in \mathbf{C}$,

$$
\operatorname{dim}(S)=\liminf _{n \rightarrow \infty} \frac{K(S \uparrow n)}{n}
$$

and

$$
\operatorname{Dim}(S)=\limsup _{n \rightarrow \infty} \frac{K(S\lceil n)}{n}
$$

Proof. We give the proof for dimension; the proof for strong dimension is analogous.

Let $t>\operatorname{dim}(S)$ be rational. Then by Corollary $3.12, S \in S^{\infty}\left[\mathbf{d}^{(t)}\right]$. Recall from the proof of Theorem 3.11 that $\mathbf{d}^{(t)}(w)=2^{t|w|} \mathbf{M}(w)$ for all $w \in\{0,1\}^{*}$. Since $\mathbf{d}^{(t)}$ succeeds on $S$, there is an infinite set $J$ such that $\mathbf{d}^{(t)}(S\lceil n) \geq 1$ for all $n \in J$. Therefore

$$
-\log \mathbf{M}\left(S\lceil n) \leq-\log 2^{-t n}=t n\right.
$$

for all $n \in J$. As

$$
|-\log \mathbf{M}(w)-K(w)|=O(\log |w|)
$$

(see [28]), the inequality

$$
\liminf _{n \rightarrow \infty} \frac{K(S\lceil n)}{n} \leq t
$$


follows.

Now let

$$
t>s>r>\liminf _{n \rightarrow \infty} \frac{K(S \uparrow n)}{n}
$$

be rational. Then for infinitely many $n, K(S \uparrow n)<r n$. Then using $(3.1)$, there is an infinite set $J$ such that $-\log \mathbf{M}(S \uparrow n)<s n$ for all $n \in J$. Therefore

$$
\mathbf{d}^{(t)}\left(S\lceil n)=2^{t n} \mathbf{M}\left(S\lceil n)>2^{(t-s) n}\right.\right.
$$

for all $n \in J$, so $S \in S^{\infty}[\mathbf{d}(t)]$ and $\operatorname{dim}(S) \leq t$.

Note that any of the usual variants of Kolmogorov complexity can be used in Theorem 3.14 as they all coincide up to a logarithmic additive term.

The rate at which a gambler can increase its capital when betting in a given situation is a fundamental concern of classical and algorithmic information and computational learning theories. In the setting of constructive gamblers, the following quantities are of particular relevance.

Definition. Let $d$ be a supermartingale, let $S \in \mathbf{C}$, and let $X \subseteq \mathbf{C}$.

1. The lower $d$-Lyapunov exponent of $S$ is

$$
\lambda_{d}(S)=\liminf _{n \rightarrow \infty} \frac{\log d(S\lceil n)}{n} .
$$

2. The upper $d$-Lyapunov exponent of $S$ is

$$
\Lambda_{d}(S)=\limsup _{n \rightarrow \infty} \frac{\log d(S\lceil n)}{n} .
$$

3. The lower Lyapunov exponent of $S$ is

$$
\lambda(S)=\sup \left\{\lambda_{d}(S) \mid d \text { is a constructive supermartingale }\right\} .
$$


4. The upper Lyapunov exponent of $S$ is

$$
\Lambda(S)=\sup \left\{\Lambda_{d}(S) \mid d \text { is a constructive supermartingale }\right\}
$$

5. The lower Lyapunov exponent of $X$ is

$$
\lambda(X)=\inf _{S \in X} \lambda(S)
$$

6. The upper Lyapunov exponent of $X$ is

$$
\Lambda(X)=\inf _{S \in X} \Lambda(S)
$$

Lyapunov exponents such as these were investigated by Schnorr [53, 55], Ryabko [51], and Staiger $[57,58]$ (using slightly different notations) prior to the effectivization of Hausdorff dimension. The quantities $\lambda_{d}(S)$ and $\Lambda_{d}(S)$ are also called "exponents of increase" of $d$ on $S$. It is implicit in Staiger's paper [57] that

$$
\Lambda_{\text {comp }}(S)=1-\operatorname{dim}_{\text {comp }}(S)
$$

for all $S \in \mathbf{C}$, where $\Lambda_{\text {comp }}(S)$ is defined like $\Lambda(S)$ above, but with $d$ required to be a computable martingale. Similar reasoning leads to the following characterizations of the Lyapunov exponents.

Theorem 3.15. Let $S \in \mathbf{C}$ and $X \subseteq \mathbf{C}$. Then $\Lambda(S)=1-\operatorname{dim}(S), \lambda(S)=1-\operatorname{Dim}(S)$, $\Lambda(X)=1-\operatorname{cdim}(X)$, and $\lambda(X)=1-\operatorname{cDim}(X)$.

Proof. We show that $\Lambda(S)=1-\operatorname{dim}(S)$. A similar argument shows that $\lambda(S)=1-\operatorname{Dim}(S)$. By Theorem 3.13, $\Lambda(X)=1-\operatorname{cdim}(X)$ and $\lambda(X)=1-\operatorname{cDim}(X)$ follow from the statements about sequences.

Let $t<s<\Lambda(S)$ with $t$ computable, and let $d$ be a constructive martingale for which $\lambda_{d}(S)>s$. Then for infinitely many $n, d\left(S\lceil n)>2^{s n}\right.$. Define a constructive $(1-t)-$ 
gale $d^{\prime}$ by $d^{\prime}(w)=2^{-t|w|} d(w)$ for all $w \in\{0,1\}^{*}$. Then for infinitely many $n$, we have $d^{\prime}\left(S\lceil n)=2^{-t n} d\left(S\lceil n)>2^{(s-t) n}\right.\right.$, so $S \in S^{\infty}[d]$. Therefore $\operatorname{dim}(S)<1-t$. This holds for all computable $t<\Lambda(S)$, so $\operatorname{dim}(S) \leq 1-\Lambda(S)$.

Let $s>\operatorname{dim}(S)$ be computable, and let $d$ be a constructive $s$-gale with $S \in S^{\infty}[d]$. Define a constructive martingale $d^{\prime}$ by $d^{\prime}(w)=2^{(1-s)|w|} d(w)$ for all $w \in\{0,1\}^{*}$. For infinitely many $n$, we have $d\left(S\lceil n)>1\right.$, and for each of these $n, d^{\prime}\left(S\lceil n)>2^{(1-s) n}\right.$. Therefore $\Lambda_{d^{\prime}}(S) \geq 1-s$, so $\Lambda(S) \geq 1-s$. This holds for all $s>\operatorname{dim}(S)$, so $\Lambda(S) \geq 1-\operatorname{dim}(S)$.

In the classical case, Tricot [61] has defined a set to be regular if its Hausdorff and packing dimensions coincide, and defined its irregularity to be the difference between these two fractal dimensions. Analogously, we define the c-irregularity (i.e., constructive irregularity) of a sequence $S \in \mathbf{C}$ to be $\operatorname{Dim}(S)-\operatorname{dim}(S)$, and we define the c-irregularity of a set $X \subseteq \mathbf{C}$ to be $\mathrm{cDim}(X)-\operatorname{cdim}(X)$. We define a sequence or set to be c-regular (i.e., constructively regular) if its c-irregularity is 0 .

As the following result shows, the c-irregularity of a sequence may be any real number in $[0,1]$

Theorem 3.16. For any two real numbers $0 \leq \alpha \leq \beta \leq 1$, there is a sequence $S \in \mathbf{C}$ such that $\operatorname{dim}(S)=\alpha$ and $\operatorname{Dim}(S)=\beta$.

Proof. Let $R$ be a Martin-Löf random sequence. It is well-known that

$$
K(R \uparrow n) \geq n-O(1)
$$

Write $R=r_{1} r_{2} r_{3} \ldots$ where $\left|r_{n}\right|=2 n-1$ for all $n$. Note that $\left|r_{1} \cdots r_{n}\right|=n^{2}$.

For each $n$, define

$$
\gamma_{n}= \begin{cases}\frac{1-\alpha}{\alpha} & \text { if } \log ^{*} n \text { is odd } \\ \frac{1-\beta}{\beta} & \text { if } \log ^{*} n \text { is even }\end{cases}
$$

and let

$$
k_{n}=\left\lceil\left|r_{n}\right| \gamma_{n}\right\rceil
$$


We now define $S \in \mathbf{C}$ as

$$
S=r_{1} 0^{k_{1}} r_{2} 0^{k_{2}} \cdots r_{n} 0^{k_{n}} \cdots
$$

Note that for all $n$,

$$
\begin{aligned}
\left|r_{n} 0^{k_{n}}\right| & =\left\lceil\left|r_{n}\right|\left(1+\gamma_{n}\right)\right\rceil \\
& = \begin{cases}\left\lceil\frac{1}{\alpha}\left|r_{n}\right|\right\rceil & \text { if } \log ^{*} n \text { is odd } \\
\left\lceil\frac{1}{\beta}\left|r_{n}\right|\right] & \text { if } \log ^{*} n \text { is even }\end{cases}
\end{aligned}
$$

Let $w \sqsubseteq S$. Then for some $n$,

$$
w=r_{1} 0^{k_{1}} \cdots r_{n-1} 0^{k_{n-1}} r_{n}^{\prime} 0^{j}
$$

where $r_{n}^{\prime} \sqsubseteq r_{n}$ and $0 \leq j \leq k_{n}$. We have

$$
\begin{aligned}
K(w) & \leq K\left(r_{1} \cdots r_{n-1} r_{n}^{\prime}\right)+K\left(k_{1}\right)+\cdots K\left(k_{n-1}\right)+K(j)+O(1) \\
& \leq\left|r_{1} \cdots r_{n-1} r_{n}^{\prime}\right|+O(n \log n) \\
& \leq(n-1)^{2}+O(n \log n) .
\end{aligned}
$$

Also,

$$
\begin{aligned}
K\left(r_{1} \cdots r_{n-1} r_{n}^{\prime}\right) & \leq K(w)+K\left(k_{1}\right)+\cdots+K\left(k_{n-1}\right)+K(j)+O(1) \\
& \leq K(w)+O(n \log n)
\end{aligned}
$$

so by $(3.2)$,

$$
\begin{aligned}
K(w) & \geq K\left(r_{1} \cdots r_{n-1} r_{n}^{\prime}\right)-O(n \log n) \\
& \geq\left|r_{1} \cdots r_{n-1} r_{n}^{\prime}\right|-O(n \log n) \\
& \geq(n-1)^{2}-O(n \log n)
\end{aligned}
$$


We bound the length of $w$ in terms of $n$ as

$$
\begin{aligned}
|w| & \geq\left|r_{1}\right|\left(1+\gamma_{1}\right)+\cdots+\left|r_{n-1}\right|\left(1+\gamma_{n-1}\right)+\left|r_{n}^{\prime}\right| \\
& \geq \frac{\left|r_{1} \cdots r_{n-1}\right|}{\beta} \\
& =\frac{1}{\beta}(n-1)^{2}
\end{aligned}
$$

and

$$
\begin{aligned}
|w| & \leq\left|r_{1}\right|\left(1+\gamma_{1}\right)+\cdots+\left|r_{n-1}\right|\left(1+\gamma_{n-1}\right)+\left|r_{n}\right|\left(1+\gamma_{n}\right)+n \\
& \leq \frac{\left|r_{1} \cdots r_{n-1} r_{n}\right|}{\alpha}+n \\
& \leq \frac{1}{\alpha}(n+1)^{2} .
\end{aligned}
$$

From (3.3) and (3.5), we have

$$
\limsup _{m \rightarrow \infty} \frac{K(S \uparrow m)}{m} \leq \limsup _{n \rightarrow \infty} \frac{(n-1)^{2}+O(n \log n)}{\frac{1}{\beta}(n-1)^{2}}=\beta,
$$

and (3.4) and (3.6) yield

$$
\liminf _{m \rightarrow \infty} \frac{K(S \uparrow m)}{m} \geq \liminf _{n \rightarrow \infty} \frac{(n-1)^{2}-O(n \log n)}{\frac{1}{\alpha}(n+1)^{2}}=\alpha .
$$

For each $n$, let

$$
w_{n}=r_{1} 0^{k_{1}} \cdots r_{n} 0^{k_{n}}
$$

Recall the sequence of towers defined by $t_{j}$ by $t_{0}=1$ and $t_{j+1}=2^{t_{j}}$. If $j$ is even, then for all 
$t_{j-1}<i \leq t_{j}, \gamma_{i}=\frac{1-\beta}{\beta}$. Then

$$
\begin{aligned}
\left|w_{t_{j}}\right| & \leq t_{j}+\sum_{i=1}^{t_{j}}\left|r_{i}\right|\left(1+\gamma_{i}\right) \\
& =t_{j}+\sum_{i=1}^{t_{j-1}}\left|r_{i}\right|\left(1+\gamma_{i}\right)+\frac{1}{\beta} \sum_{i=t_{j-1}+1}^{t_{j}}\left|r_{i}\right| \\
& \leq t_{j}+\frac{1}{\alpha} t_{j-1}^{2}+\frac{1}{\beta}\left(t_{j}^{2}-t_{j-1}^{2}\right) \\
& \leq \frac{1}{\beta} t_{j}^{2}+t_{j}+O\left(\left(\log t_{j}\right)^{2}\right) .
\end{aligned}
$$

Similarly, if $j$ is odd, we have

$$
\begin{aligned}
\left|w_{t_{j}}\right| & \geq \sum_{i=1}^{t_{j}}\left|r_{i}\right|\left(1+\gamma_{i}\right) \\
& =\sum_{i=1}^{t_{j-1}}\left|r_{i}\right|\left(1+\gamma_{i}\right)+\frac{1}{\alpha} \sum_{i=t_{j-1}+1}^{t_{j}}\left|r_{i}\right| \\
& \geq \frac{1}{\beta} t_{j-1}^{2}+\frac{1}{\alpha}\left(t_{j}^{2}-t_{j-1}^{2}\right) \\
& \geq \frac{1}{\alpha} t_{j}^{2}-O\left(\left(\log t_{j}\right)^{2}\right) .
\end{aligned}
$$

Combining (3.4) and (3.9), we have

$$
\limsup _{m \rightarrow \infty} \frac{K(S \uparrow m)}{m} \geq \limsup _{n \rightarrow \infty} \frac{K\left(w_{t_{2 n}}\right)}{\left|w_{t_{2 n}}\right|} \geq \beta
$$

Putting (3.3) together with (3.10) yields

$$
\liminf _{m \rightarrow \infty} \frac{K(S \uparrow m)}{m} \leq \liminf _{n \rightarrow \infty} \frac{K\left(w_{t_{2 n+1}}\right)}{\left|w_{t_{2 n+1}}\right|} \leq \alpha .
$$

By Theorem 3.14, (3.8), and (3.12), we have $\operatorname{dim}(S)=\alpha$. We also have $\operatorname{Dim}(S)=\beta$ from Theorem 3.14, (3.7), and (3.11).

Examples of c-regular sequences that are more natural than those given by Theorem 3.16 can be obtained by generalizing the construction of Chaitin's random real number $\Omega[11]$. 
Mayordomo [43] and, independently, Tadaki [60] defined for each $s \in(0,1]$ and each infinite, computably enumerable set $A \subseteq\{0,1\}^{*}$, the real number

$$
\theta_{A}^{s}=\sum\left\{2^{\frac{|\pi|}{s}} \mid \pi \in\{0,1\}^{*} \text { and } U(\pi) \in A\right\}
$$

where $U$ is a universal self-delimiting Turing machine. Given Theorem 3.14, the following fact is implicit in Tadaki's paper.

Theorem 3.17. (Tadaki [60]) For each $s \in(0,1]$ and each infinite, computably enumerable set $A \subseteq\{0,1\}^{*}$, the (binary expansion of the) real number $\theta_{A}^{s}$ is c-regular with $\operatorname{dim}\left(\theta_{A}^{s}\right)=$ $\operatorname{Dim}\left(\theta_{A}^{s}\right)=s$.

\subsubsection{Gales Suffice for Constructive Dimension}

In [35], a conference paper preceding [31], Lutz defined constructive dimension using constructive gales. There Lutz used an incorrect assertion about martingales to argue that for each computable $s$ there exists a constructive $s$-gale that is multiplicatively optimal for the class of constructive $s$-gales. These "optimal gales" were then used to prove Theorem 3.13. These flawed arguments were subsequently noticed and corrected in [31] by reformulating constructive dimension in terms of constructive supergales. The multiplicatively optimal supergales of Theorem 3.11 exist and Theorem 3.13 is true in the reformulation. However, Lutz left open the questions of whether there exist optimal constructive gales and whether constructive dimension can be equivalently defined using constructive gales. We now address these questions.

Theorem 3.18. Let $0 \leq r<t$ be computable real numbers. Then for any constructive $r$ supergale $d$, there exists a constructive t-gale $d^{\prime}$ such that $S^{\infty}[d] \subseteq S^{\infty}\left[d^{\prime}\right]$ and $S_{\mathrm{str}}^{\infty}[d] \subseteq S_{\mathrm{str}}^{\infty}\left[d^{\prime}\right]$.

Proof. Let $d$ be a constructive $r$-supergale, and assume without loss of generality that $d(\lambda)<$ 1. Define the language $A=\left\{w \in\{0,1\}^{*} \mid d(w)>1\right\}$. Observe that $A$ is computably enumer- 
able. For all $n \in \mathbb{N}$,

$$
\sum_{w \in\{0,1\}^{n}} d(w) \leq 2^{r n}
$$

so $\left|A_{=n}\right| \leq 2^{r n}$.

For each $n \in \mathbb{N}$, define a function $d_{n}^{\prime}:\{0,1\}^{*} \rightarrow[0, \infty)$ by

$$
d_{n}^{\prime}(w)= \begin{cases}2^{-t(n-|w|)} \cdot\left|\left\{v \in A_{=n} \mid w \sqsubseteq v\right\}\right| & \text { if }|w| \leq n \\ 2^{(t-1)(|w|-n)} d_{n}^{\prime}(w\lceil n) & \text { if }|w|>n\end{cases}
$$

Then for all $n, d_{n}^{\prime}$ is a $t$-gale and $d_{n}^{\prime}(w)=1$ for all $w \in A_{=n}$.

Let $s \in(r, t)$ be computable and define a function $d^{\prime}$ on $\{0,1\}^{*}$ by

$$
d^{\prime}=\sum_{n=0}^{\infty} 2^{(s-r) n} d_{n}^{\prime}
$$

Then

$$
d^{\prime}(\lambda)=\sum_{n=0}^{\infty} 2^{(s-r) n} 2^{-t n}\left|A_{=n}\right| \leq \sum_{n=0}^{\infty} 2^{(s-t) n}<\infty,
$$

and it follows by induction that $d^{\prime}(w)<\infty$ for all strings $w$. Therefore, by linearity, $d^{\prime}$ is a $t$-gale. Also, because the language $A$ is computably enumerable, $d^{\prime}$ is constructive.

For all $w \in A$,

$$
d^{\prime}(w) \geq 2^{(s-r)|w|} d_{|w|}^{\prime}(w)=2^{(s-r)|w|} .
$$

If $S \in S^{\infty}[d]$, then $S$ has infinitely many prefixes in $A$, so it follows that $S \in S^{\infty}\left[d^{\prime}\right]$. Similarly, if $S \in S_{\mathrm{str}}^{\infty}[d]$, then all but finitely many prefixes of $S$ are in $A$, so $S \in S_{\mathrm{str}}^{\infty}\left[d^{\prime}\right]$.

Constructive dimension and constructive strong dimension may now be equivalently defined using gales instead of supergales.

Theorem 3.19. For all $X \subseteq \mathbf{C}$,

$$
\operatorname{cdim}(X)=\inf \mathcal{G}_{\text {constr }}(X)
$$


and

$$
\operatorname{cDim}(X)=\inf \mathcal{G}_{\text {constr }}^{\text {str }}(X)
$$

Proof. Because any gale is also a supergale, $\mathcal{G}_{\text {constr }}(X) \subseteq \widehat{\mathcal{G}}_{\text {constr }}(X)$, so

$$
\operatorname{cdim}(X)=\inf \widehat{\mathcal{G}}_{\text {constr }}(X) \leq \inf \mathcal{G}_{\text {constr }}(X)
$$

is immediate.

Let $t>r>\operatorname{cdim}(X)$ be computable real numbers and let $d$ be a constructive $r$-supergale such that $X \subseteq S^{\infty}[d]$. By Theorem 3.18, there is a constructive $t$-gale $d^{\prime}$ such that $X \subseteq$ $S^{\infty}[d] \subseteq S^{\infty}\left[d^{\prime}\right]$, so $t \in \mathcal{G}_{\text {constr }}(X)$. As this holds for any computable $t>\operatorname{cdim}(X)$, we have $\inf \mathcal{G}_{\text {constr }}(X) \leq \operatorname{cdim}(X)$.

The proof for constructive strong dimension is analogous.

We can also state the existence of a form of optimal constructive gales.

Definition. Let $d^{*}$ be a supergale and let $\mathcal{D}$ be a class of supergales. We say that $d^{*}$ is success-optimal for $\mathcal{D}$ if for every $d \in \mathcal{D}, S^{\infty}[d] \subseteq S^{\infty}\left[d^{*}\right]$ and $S_{\mathrm{str}}^{\infty}[d] \subseteq S_{\mathrm{str}}^{\infty}\left[d^{*}\right]$.

Corollary 3.20. For all computable real numbers $t>r \geq 0$ there exists a constructive $t$-gale that is success-optimal for the class of constructive r-supergales.

Proof. Let $\mathbf{d}^{(r)}$ be the constructive $r$-supergale from Theorem 3.11 that is multiplicatively optimal for the constructive $r$-supergales. Take the constructive $t$-gale $d^{\prime}$ from Theorem 3.18 that succeeds everywhere that $\mathbf{d}^{(r)}$ does. Therefore $S^{\infty}[d] \subseteq S^{\infty}\left[\mathbf{d}^{(r)}\right] \subseteq S^{\infty}\left[d^{\prime}\right]$ (and similarly for strong success) for any constructive $r$-supergale $d$, so the corollary is proved.

The optimal gales provided by Corollary 3.20 may not be technically as strong as possible, in two respects.

1. Lutz's optimal constructive $r$-supergale is multiplicatively optimal, whereas our optimal constructive $t$-gale is only success-optimal. Does there exist a constructive $t$-gale that is multiplicatively optimal for the class of constructive $r$-supergales? 
2. Our proof seems to require the hypothesis $t>r$. Does there exist a constructive $r$-gale that is success-optimal for the class of constructive $r$-supergales?

However, the optimality in Corollary 3.20 remains strong enough to directly prove Theorem 3.13 using the gale definition of Theorem 3.19.

\subsubsection{Entropy Rates}

In this section we show that the constructive dimensions characterize entropy rates of the type investigated by Staiger $[56,57]$.

Definition. Let $A \subseteq\{0,1\}^{*}$.

1. The entropy rate of $A \subseteq\{0,1\}^{*}$ is

$$
H_{A}=\limsup _{n \rightarrow \infty} \frac{\log \left|A_{=n}\right|}{n} .
$$

2. We define the sets of sequences

$$
A^{\text {i.o. }}=\left\{S \in \mathbf{C} \mid\left(\exists^{\infty} n\right) S \uparrow n \in A\right\}
$$

and

$$
A^{\text {a.e. }}=\left\{S \in \mathbf{C} \mid\left(\forall^{\infty} n\right) S \uparrow n \in A\right\} .
$$

In $[56,57], A^{\text {i.o. }}$ is called $A^{\delta}$, the $\delta$-limit of $A$. Staiger observed that $H_{A}$ has a useful alternate characterization.

Lemma 3.21. (Staiger [56]) For any $A \subseteq\{0,1\}^{*}$,

$$
H_{A}=\inf \left\{s \mid \sum_{w \in A} 2^{-s|w|}<\infty\right\} .
$$

Proof. Let

$$
r=\inf \left\{s \mid \sum_{w \in A} 2^{-s|w|}<\infty\right\} .
$$


Let $s>H_{A}$ and $\epsilon=s-H_{A}$. Then for some $n_{0}$, we have

$$
H_{A}+\frac{\epsilon}{2}>\frac{\log \left|A_{=n}\right|}{n}
$$

for all $n \geq n_{0}$. We have

$$
\sum_{w \in A} 2^{-s|w|}=\sum_{w \in A} 2^{-\left(H_{A}+\epsilon\right)|w|}=\sum_{n=0}^{\infty}\left|A_{=n}\right| 2^{-\left(H_{A}+\epsilon\right) n}
$$

and

$$
\begin{aligned}
\sum_{n=n_{0}}^{\infty}\left|A_{=n}\right| 2^{-\left(H_{A}+\frac{\epsilon}{2}\right) n} 2^{-\frac{\epsilon}{2} n} & <\sum_{n=n_{0}}^{\infty}\left|A_{=n}\right| 2^{-\left(\frac{\log |A=n|}{n}\right) n} 2^{-\frac{\epsilon}{2} n} \\
& =\sum_{n=n_{0}}^{\infty} 2^{-\frac{\epsilon}{2} n} \\
& <\infty
\end{aligned}
$$

so

$$
\sum_{w \in A} 2^{-s|w|}<\infty
$$

Therefore $r \leq s$. As $s>H_{A}$ is arbitrary, we have $r \leq H_{A}$.

For the other inequality, let $s>r$. Then since

$$
\sum_{n=0}^{\infty} 2^{-s n}\left|A_{=n}\right|=\sum_{w \in A} 2^{-s|w|}<\infty
$$

there is some $n_{0}$ such that $2^{-s n}\left|A_{=n}\right| \leq 1$ for all $n \geq n_{0}$. Therefore

$$
\frac{\log \left|A_{=n}\right|}{n} \leq s
$$

for all $n \geq n_{0}$, so $H_{A} \leq s$. Since $s>r$ is arbitrary, we have $H_{A} \leq r$.

We now give a general definition of entropy rate of a class of sequences that takes a class of languages as a parameter. 
Definition. Let $X \subseteq \mathbf{C}$ and let $\mathcal{C}$ be a class of languages.

1. The $\mathcal{C}$-entropy rate of $X$ is

$$
\mathcal{H}_{\mathcal{C}}(X)=\inf \left\{H_{A} \mid X \subseteq A^{\text {i.o. }} \text { and } A \in \mathcal{C}\right\}
$$

2. The strong $\mathcal{C}$-entropy rate of $X$ is

$$
\mathcal{H}_{\mathcal{C}}^{\text {str }}(X)=\inf \left\{H_{A} \mid X \subseteq A^{\text {a.e. }} \text { and } A \in \mathcal{C}\right\}
$$

For now we are interested in the cases when $\mathcal{C}=\mathrm{ALL}$, the class of all languages, and $\mathcal{C}=\mathrm{CE}$, the class of computably enumerable languages. In Section 3.3.2 the PSPACE- and DECentropy rates will also be of interest.

The classical Hausdorff and packing dimensions may be characterized in terms of entropy rates.

Theorem 3.22. For any $X \subseteq \mathbf{C}$,

$$
\operatorname{dim}_{\mathrm{H}}(X)=\mathcal{H}_{\mathrm{ALL}}(X)
$$

and

$$
\operatorname{dim}_{\mathrm{P}}(X)=\mathcal{H}_{\mathrm{ALL}}^{\mathrm{str}}(X)
$$

Proof. A proof of the Hausdorff dimension part can be found in [56]; it also follows from Theorem 32 of [47]. We omit a proof for packing dimension. (Both parts can also be proved using the arguments from the proofs of Lemmas 3.23 and 3.24 below.)

In analogy with Theorem 3.22 we will show that the constructive dimensions can be characterized using the constructive entropy rates $\mathcal{H}_{\mathrm{CE}}(X)$ and $\mathcal{H}_{\mathrm{CE}}^{\mathrm{str}}(X)$. First we show that the dimensions are lower bounds of the entropy rates. 
Lemma 3.23. For any $X \subseteq \mathbf{C}$,

$$
\operatorname{cdim}(X) \leq \mathcal{H}_{\mathrm{CE}}(X)
$$

and

$$
\mathrm{cDim}(X) \leq \mathcal{H}_{\mathrm{CE}}^{\mathrm{str}}(X)
$$

Proof. Let $A \subseteq\{0,1\}^{*}$ and let $t>s>H_{A}$. For each $n \in \mathbb{N}$, define a function $d_{n}:\{0,1\}^{*} \rightarrow$ $[0, \infty)$ by

$$
d_{n}(w)= \begin{cases}2^{-t(n-|w|)} \cdot\left|\left\{v \in A_{=n} \mid w \sqsubseteq v\right\}\right| & \text { if }|w| \leq n \\ 2^{(t-1)(|w|-n)} d_{n}(w\lceil n) & \text { if }|w|>n .\end{cases}
$$

Then each $d_{n}$ is a $t$-gale. Define a function $d$ on $\{0,1\}^{*}$ by $d=\sum_{n=0}^{\infty} 2^{(t-s) n} d_{n}$. Then

$$
d(\lambda)=\sum_{n=0}^{\infty} 2^{(t-s) n} 2^{-t n}\left|A_{=n}\right|=\sum_{w \in A} 2^{-s|w|}<\infty
$$

because $s>H_{A}$. By induction, $d(w)<\infty$ for all strings $w$, so $d:\{0,1\}^{*} \rightarrow[0, \infty)$. By linearity, $d$ is also a $t$-gale. For any $w \in A$, we have

$$
d(w) \geq 2^{(t-s)|w|} d_{|w|}(w)=2^{(t-s)|w|},
$$

so it follows that $A^{\text {i.o. }} \subseteq S^{\infty}[d]$ and $A^{\text {a.e. }} \subseteq S_{\text {str }}^{\infty}[d]$.

Let $r>\mathcal{H}_{\mathrm{CE}}(X)$ be arbitrary. Then there is a computably enumerable $A$ with $X \subseteq A^{\text {i.o. }}$ and $H_{A}<r$. We can also choose $t$ and $s$ computable so that $H_{A}<s<t<r$. Because $A$ is computably enumerable, the $t$-gale $d$ defined above is constructive. Since $X \subseteq A^{\text {i.o. }} \subseteq S^{\infty}[d]$, we have $\operatorname{cdim}(X) \leq t<r$. As this holds for all $r>\mathcal{H}_{\mathrm{CE}}(X)$, we have $\operatorname{cdim}(X) \leq \mathcal{H}_{\mathrm{CE}}(X)$.

The proof that $\mathrm{cDim}(X) \leq \mathcal{H}_{\mathrm{CE}}^{\mathrm{str}}(X)$ is analogous to the previous paragraph.

Next we give lower bounds for the dimensions by entropy rates. 
Lemma 3.24. For all $X \subseteq \mathbf{C}$,

$$
\mathcal{H}_{\mathrm{CE}}(X) \leq \operatorname{cdim}(X)
$$

and

$$
\mathcal{H}_{\mathrm{CE}}^{\mathrm{str}}(X) \leq \mathrm{cDim}(X)
$$

Proof. Suppose that $d$ is an $s$-supergale with $X \subseteq S^{\infty}[d]$. Assume without loss of generality that $d(\lambda)<1$ and let $A=\{w \mid d(w)>1\}$. Then for all $n \in \mathbb{N}$,

$$
\sum_{w \in\{0,1\}^{n}} d(w) \leq 2^{s n}
$$

and $\left|A_{=n}\right| \leq 2^{s n}$. Also, $X \subseteq S^{\infty}[d] \subseteq A^{\text {i.o. }}$. For any $t>s$,

$$
\sum_{w \in A} 2^{-t|w|}=\sum_{n=0}^{\infty} 2^{-t n}\left|A_{=n}\right| \leq \sum_{n=0}^{\infty} 2^{(s-t) n}<\infty
$$

so $H_{A} \leq t$. Therefore $H_{A} \leq s$.

If we let $s>\operatorname{cdim}(X)$ be computable, then there is a constructive $s$-supergale $d$ with $X \subseteq S^{\infty}[d]$. Then the set $A$ defined above is computably enumerable and $X \subseteq A^{\text {i.o. }}$, so $\mathcal{H}_{\mathrm{CE}}(X) \leq H_{A}$. We showed that $H_{A} \leq s$, so $\mathcal{H}_{\mathrm{CE}}(X) \leq s$. Therefore $\mathcal{H}_{\mathrm{CE}}(X) \leq \operatorname{cdim}(X)$.

The proof that $\mathcal{H}_{\mathrm{CE}}^{\mathrm{str}}(X) \leq \mathrm{cDim}(X)$ is analogous.

We now have an equivalence between constructive entropy rates and the constructive dimensions.

Theorem 3.25. For all $X \subseteq \mathbf{C}$,

$$
\operatorname{cdim}(X)=\mathcal{H}_{\mathrm{CE}}(X)
$$

and

$$
\operatorname{cDim}(X)=\mathcal{H}_{\mathrm{CE}}^{\mathrm{str}}(X)
$$


Proof. This follows immediately from Lemmas 3.23 and 3.24.

\subsection{Resource-Bounded Dimensions}

In this section we define resource-bounded dimension and resource-bounded strong dimension. Resource-bounded dimension was introduced by Lutz [30] by effectivizing the gale characterization of Hausdorff dimension (Theorem 3.5) in much the same way that the martin-

gale characterization of Lebesgue measure (Theorem 3.3) was effectivized to define resourcebounded measure [33].

Unless otherwise specified, $\Delta$ denotes any of the resource bounds defined in Chapter 2. We begin by recalling the definition of resource-bounded measure. This is done by restricting the martingales in Theorem 3.3 to be $\Delta$-computable.

Definition. Let $X \subseteq \mathbf{C}$.

1. We say that $X$ has $\Delta$-measure 0 , and write $\mu_{\Delta}(X)=0$, if there is a $\Delta$-computable martingale $d$ such that $X \subseteq S^{\infty}[d]$.

2. We say that $X$ has $\Delta$-measure 1 , and write $\mu_{\Delta}(X)=1$, if $\mu_{\Delta}\left(X^{c}\right)=0$.

3. We say that $X$ has measure 0 in $R(\Delta)$, and write $\mu(X \mid R(\Delta))=0$, if $\mu_{\Delta}(X \cap R(\Delta))=0$.

4. We say that $X$ has measure 1 in $R(\Delta)$, and write $\mu(X \mid R(\Delta))=1$, if $\mu_{\Delta}\left(X^{c} \mid R(\Delta)\right)=$ 0.

The definition of measure in $R(\Delta)$ is justified by the following theorem.

Theorem 3.26. (Measure Conservation Theorem $(\operatorname{Lutz}[33])) \mu_{\Delta}(R(\Delta)) \neq 0$.

In particular, Theorem 3.26 implies that the conditions $\mu_{\Delta}(X \mid R(\Delta))=0$ and $\mu_{\Delta}(X \mid$ $R(\Delta))=1$ are mutually exclusive.

To effectivize the Hausdorff and packing dimensions, we use $\Delta$-computable gales and supergales. The following sets are similar to those defined in Section 3.2 that were used for the constructive dimensions. 
Notation. Let $X \subseteq \mathbf{C}$.

1. $\mathcal{G}_{\Delta}(X)$ is the set of all $s \in[0, \infty)$ for which there exists a $\Delta$-computable $s$-gale $d$ such that $X \subseteq S^{\infty}[d]$.

2. $\mathcal{G}_{\Delta}^{\operatorname{str}}(X)$ is the set of all $s \in[0, \infty)$ for which there exists a $\Delta$-computable $s$-gale $d$ such that $X \subseteq S_{\text {str }}^{\infty}[d]$.

3. $\widehat{\mathcal{G}}_{\Delta}(X)$ is the set of all $s \in[0, \infty)$ for which there exists a $\Delta$-computable $s$-supergale $d$ such that $X \subseteq S^{\infty}[d]$.

4. $\widehat{\mathcal{G}}_{\Delta}^{\mathrm{str}}(X)$ is the set of all $s \in[0, \infty)$ for which there exists a $\Delta$-computable $s$-supergale $d$ such that $X \subseteq S_{\mathrm{str}}^{\infty}[d]$.

The following effectivizations of Hausdorff and packing dimension are motivated by Theorems 3.5 and 3.6 .

Definition. Let $X \subseteq \mathbf{C}$ and $S \in \mathbf{C}$.

1. The $\Delta$-dimension of $X$ is

$$
\operatorname{dim}_{\Delta}(X)=\inf \mathcal{G}_{\Delta}(X)
$$

2. The $\Delta$-strong dimension of $X$ is

$$
\operatorname{Dim}_{\Delta}(X)=\inf \mathcal{G}_{\Delta}^{\operatorname{str}}(X)
$$

3. The dimension of $X$ in $R(\Delta)$ is

$$
\operatorname{dim}(X \mid R(\Delta))=\operatorname{dim}_{\Delta}(X \cap R(\Delta)) .
$$

4. The strong dimension of $X$ in $R(\Delta)$ is

$$
\operatorname{Dim}(X \mid R(\Delta))=\operatorname{Dim}_{\Delta}(X \cap R(\Delta)) .
$$


The polynomial-time dimensions $\operatorname{dim}_{\mathrm{p}}(X)$ and $\operatorname{Dim}_{\mathrm{p}}(X)$ are also called the feasible dimension and the feasible strong dimension, respectively. The notation $\operatorname{dim}_{\mathrm{p}}(X)$ for the $\mathrm{p}$ dimension is all too similar to the notation $\operatorname{dim}_{\mathrm{P}}(X)$ for the classical packing dimension, but confusion is unlikely because these dimensions typically arise in quite different contexts.

Note that the classical Hausdorff and packing dimensions can each now be written in three different ways, i.e.,

$$
\operatorname{dim}_{\mathrm{H}}(X)=\operatorname{dim}_{\text {all }}(X)=\operatorname{dim}(X \mid \mathbf{C})
$$

and

$$
\operatorname{dim}_{\mathrm{P}}(X)=\operatorname{Dim}_{\text {all }}(X)=\operatorname{Dim}(X \mid \mathbf{C})
$$

In the definition of the resource-bounded dimensions, we could equivalently use the "hatted" sets $\widehat{\mathcal{G}}_{\Delta}(X)$ and $\widehat{\mathcal{G}}_{\Delta}^{\text {str }}(X)$ in place of their unhatted counterparts. This is immediate from the following exact computation lemma.

Lemma 3.27. (Lutz [30]) If $d$ is a $\Delta$-computable s-supergale and $2^{s}$ is rational, then there is an exactly $\Delta$-computable s-gale $\tilde{d}$ such that $S^{\infty}[d] \subseteq S^{\infty}[\tilde{d}]$ and $S_{\mathrm{str}}^{\infty}[d] \subseteq S_{\mathrm{str}}^{\infty}[\tilde{d}]$.

We now give some basic properties of the resource-bounded dimensions.

Observations 3.28. Let $X, Y \subseteq \mathbf{C}$ and let $\Delta, \Delta^{\prime}$ be resource-bounds.

1. $0 \leq \operatorname{dim}_{\Delta}(X) \leq \operatorname{Dim}_{\Delta}(X) \leq 1$.

2. If $X \subseteq Y$, then $\operatorname{dim}_{\Delta}(X) \leq \operatorname{dim}_{\Delta}(Y)$ and $\operatorname{Dim}_{\Delta}(X) \leq \operatorname{Dim}_{\Delta}(Y)$.

3. If $\Delta \subseteq \Delta^{\prime}$, then $\operatorname{dim}_{\Delta^{\prime}}(X) \leq \operatorname{dim}_{\Delta}(X)$ and $\operatorname{Dim}_{\Delta^{\prime}}(X) \leq \operatorname{Dim}_{\Delta}(X)$.

Resource-bounded dimension refines resource-bounded measure in the following way.

Proposition 3.29. (Lutz [30]) Let $X \subseteq \mathbf{C}$.

1. If $\operatorname{dim}_{\Delta}(X)<1$, then $\mu_{\Delta}(X)=0$.

2. If $\operatorname{dim}(X \mid R(\Delta))<1$, then $\mu(X \mid R(\Delta))=0$. 
Proof. Assume that $\operatorname{dim}_{\Delta}(X)<1$ and let $d$ be a $\Delta$-computable $s$-gale with $X \subseteq S^{\infty}[d]$ and $s<1$. Then $d$ is a 1 -supergale, so by Lemma 3.27 , there is an exactly $\Delta$-computable martingale (i.e., a 1-gale) $\tilde{d}$ with $S^{\infty}[d] \subseteq S^{\infty}[\tilde{d}]$, so $\mu_{\Delta}(X)=0$.

That is, sets that do not have $\Delta$-measure 0 have $\Delta$-dimension 1 . In particular, from Theorem 3.26, we have the following.

Corollary 3.30. (Lutz [30])

1. $\operatorname{dim}_{\mathrm{p}}(\mathrm{E})=\operatorname{Dim}_{\mathrm{p}}(\mathrm{E})=1$.

2. $\operatorname{dim}_{\mathrm{p}_{2}}(\mathrm{EXP})=\operatorname{Dim}_{\mathrm{p}_{2}}(\mathrm{EXP})=1$.

3. $\operatorname{dim}_{\mathrm{pspace}}(\mathrm{ESPACE})=\operatorname{Dim}_{\mathrm{pspace}}(\mathrm{ESPACE})=1$.

4. $\operatorname{dim}_{\mathrm{p}_{2} \text { space }}(\mathrm{EXPSPACE})=\operatorname{Dim}_{\mathrm{P}_{2} \text { space }}(\mathrm{EXPSPACE})=1$.

On the other hand, "slices" of these classes have $\Delta$-dimension 0 .

Proposition 3.31. (Lutz [30]) Let $c \in \mathbb{N}$.

1. $\operatorname{dim}_{\mathrm{p}}\left(\operatorname{DTIME}\left(2^{c n}\right)=\operatorname{Dim}_{\mathrm{p}}\left(\operatorname{DTIME}\left(2^{c n}\right)\right)=0\right.$.

2. $\operatorname{dim}_{\mathrm{p}_{2}}\left(\operatorname{DTIME}\left(2^{n^{c}}\right)=\operatorname{Dim}_{\mathrm{p}_{2}}\left(\operatorname{DTIME}\left(2^{n^{c}}\right)\right)=0\right.$.

3. $\operatorname{dim}_{\text {pspace }}\left(\operatorname{DSPACE}\left(2^{c n}\right)=\operatorname{Dim}_{\text {pspace }}\left(\operatorname{DSPACE}\left(2^{c n}\right)\right)=0\right.$.

4. $\operatorname{dim}_{\mathrm{p}_{2} \text { space }}\left(\operatorname{DSPACE}\left(2^{n^{c}}\right)=\operatorname{Dim}_{\mathrm{p}_{2} \text { space }}\left(\operatorname{DSPACE}\left(2^{n^{c}}\right)\right)=0\right.$.

It is possible for a subclass of $R(\Delta)$ to have $\Delta$-dimension strictly between 0 and 1 , but so far this is not known to hold for any standard complexity class.

\subsubsection{Log-Loss Unpredictability}

In this section we establish a fundamental relationship between the Hausdorff and packing dimensions and log-loss prediction.

Consider predicting the symbols of an unknown infinite sequence. Given an initial finite segment of the sequence, a predictor specifies a probability distribution over $\{0,1\}$. We may 
think of the probability that the algorithm assigns to each character as representing the predictor's confidence of that character occurring next in the sequence. Formally, we define a predictor as follows.

Definition. A function $\pi:\{0,1\}^{*} \times\{0,1\} \rightarrow[0,1]$ is a predictor if for all $w \in\{0,1\}^{*}$, $\pi(w, 0)+\pi(w, 1)=1$.

Here we interpret $\pi(w, b)$ as the predictor $\pi$ 's estimation of the likelihood that the bit immediately following the string $w$ is $b$. There is a natural correspondence between predictors and gales. (An early reference for the following type of relationship between prediction and gambling is [12].)

Notation. 1. A predictor $\pi$ induces for each $s \in[0, \infty)$ an $s$-gale $d_{\pi}^{(s)}$ defined by the recursion

$$
\begin{gathered}
d_{\pi}^{(s)}(\lambda)=1 \\
d_{\pi}^{(s)}(w a)=2^{s} d_{\pi}^{(s)}(w) \pi(w, a)
\end{gathered}
$$

for all $w \in\{0,1\}^{*}$ and $a \in\{0,1\}$; equivalently

$$
d_{\pi}^{(s)}(w)=2^{s|w|} \prod_{i=0}^{|w|-1} \pi(w\lceil i, w[i])
$$

for all $w \in\{0,1\}^{*}$.

2. An $s$-gale $d$ with $d(\lambda)=1$ is induced by the predictor $\pi_{d}$ defined by

$$
\pi_{d}(w, a)= \begin{cases}2^{-s \frac{d(w a)}{d(w)}} & \text { if } d(w) \neq 0 \\ \frac{1}{k} & \text { otherwise }\end{cases}
$$

for all $w \in\{0,1\}^{*}$ and $a \in\{0,1\}$.

The following observation is simple but useful. 
Observation 3.32. $\quad$ 1. Let $s$ be rational and $\pi$ be a predictor. If $\pi$ is $\Delta$-computable, then $d_{\pi}^{(s)}$ is $\Delta$-computable.

2. Let $2^{s}$ be rational and $d$ be an $s$-gale. If $d$ is exactly $\Delta$-computable, then $\pi_{d}$ is exactly $\Delta$-computable.

In judging the performance of a predictor on a sequence, it is instructive to consider its "loss" on individual bits of the sequence. A standard way to do this is the log-loss function (see [44]). If the probability that the predictor assigned to the correct bit is $p$, then we assign a loss of

$$
\log \frac{1}{p}
$$

to that prediction. If $p=1$, then the loss is 0 . As $p$ approaches 0 , the loss becomes infinite. Using $p=\frac{1}{2}$ achieves a loss of 1 no matter which bit occurs.

In the following definition we write $\Pi(\Delta)$ for the class of all $\Delta$-computable predictors.

Definition. Let $w \in\{0,1\}^{*}, S \in \mathbf{C}$, and $X \subseteq \mathbf{C}$.

1. The cumulative log-loss of $\pi$ on $w$ is

$$
\mathcal{L}^{\log }(\pi, w)=\sum_{i=0}^{|w|-1} \log \frac{1}{\pi(w\lceil i, w[i])}
$$

2. The $\log$-loss rate of $\pi$ on $S$ is

$$
\mathcal{L}^{\log }(\pi, S)=\liminf _{n \rightarrow \infty} \frac{\mathcal{L}^{\log }(\pi, S\lceil n)}{n} .
$$

3. The strong log-loss rate of $\pi$ on $S$ is

$$
\mathcal{L}_{\mathrm{str}}^{\log }(\pi, S)=\limsup _{n \rightarrow \infty} \frac{\mathcal{L}^{\log }(\pi, S\lceil n)}{n}
$$


4. The (worst-case) log-loss of $\pi$ on $X$ is

$$
\mathcal{L}^{\log }(\pi, X)=\sup _{S \in X} \mathcal{L}^{\log }(\pi, S)
$$

5. The (worst-case) strong log-loss of $\pi$ on $X$ is

$$
\mathcal{L}_{\mathrm{str}}^{\log }(\pi, X)=\sup _{S \in X} \mathcal{L}_{\mathrm{str}}^{\log }(\pi, S)
$$

6. The $\Delta$-log-loss unpredictability of $X$ is

$$
\text { unpred }_{\Delta}^{\log }(X)=\inf _{\pi \in \Pi(\Delta)} \mathcal{L}^{\log }(\pi, X)
$$

7. The $\Delta$-strong log-loss unpredictability of $X$ is

$$
\operatorname{Unpred}_{\Delta}^{\log }(X)=\inf _{\pi \in \Pi(\Delta)} \mathcal{L}_{\text {str }}^{\log }(\pi, X)
$$

We now show that log-loss unpredictability equals dimension.

Theorem 3.33. For any $X \subseteq \mathbf{C}$,

$$
\operatorname{dim}_{\Delta}(X)=\operatorname{unpred}_{\Delta}^{\log }(X)
$$

and

$$
\operatorname{Dim}_{\Delta}(X)=\operatorname{Unpred}_{\Delta}^{\log }(X)
$$

Proof. Let $2^{s}$ be rational such that $\operatorname{dim}_{\Delta}(X) \leq s$ and let $d$ be an exactly $\Delta$-computable $s$-gale with $X \subseteq S^{\infty}[d]$. Assume without loss of generality that $d(\lambda)=1$. Let $\pi_{d}:\{0,1\}^{*} \times\{0,1\} \rightarrow$ $[0,1]$ be the predictor inducing $d$ as defined above. The $\pi_{d}$ is also exactly $\Delta$-computable. For 
any $w \in\{0,1\}^{*}$ with $d(w)>0$,

$$
\begin{aligned}
\mathcal{L}^{\log }\left(\pi_{d}, w\right) & =\sum_{i=0}^{|w|-1} \log \frac{1}{\pi_{d}(w\lceil i, w[i])} \\
& =-\log \prod_{i=0}^{|w|-1} \pi_{d}(w\lceil i, w[i]) \\
& =-\log 2^{-s|w|} d(w) \\
& =s|w|-\log d(w)
\end{aligned}
$$

Let $S \in S^{\infty}[d]$. Then there exist infinitely many $n \in \mathbb{N}$ such that $d(S\lceil n) \geq 1$, and for each of these $n$ we have

$$
\begin{aligned}
\frac{\mathcal{L}^{\log \left(\pi_{d}, S\lceil n)\right.}}{n} & =\frac{s n-\log d(S\lceil n)}{n} \\
& \leq \frac{s n-\log 1}{n} \\
& =s .
\end{aligned}
$$

Therefore $\mathcal{L}^{\log }\left(\pi_{d}, S\right) \leq s$, so this establishes that $\operatorname{unpred}_{\Delta}^{\log }(X) \leq \mathcal{L}^{\log }\left(\pi_{d}, X\right) \leq s$. By density of the set $\left\{s \mid 2^{s} \in \mathbb{Q}\right\}$, it follows that $\operatorname{unpred}_{\Delta}^{\log }(X) \leq \operatorname{dim}_{\Delta}(X)$. The proof that $\operatorname{Unpred}_{\Delta}^{\log }(X) \leq \operatorname{Dim}_{\Delta}(X)$ is analogous.

Now let $s>t>$ unpred $_{\Delta}^{\log }$ be rational, and let $\pi$ be a $\Delta$-computable predictor for which $\mathcal{L}_{\pi}^{\log }(X)<t$. Let $d_{\pi}^{(s)}$ be the $s$-gale induced by $\pi$ as defined above. Note that $d_{\pi}^{(s)}$ is also $\Delta$-computable. Let $S \in X$. Then there exist infinitely many $n \in \mathbb{N}$ such that

$$
\frac{\mathcal{L}_{\pi}^{\log }(S\lceil n)}{n} \leq t
$$


and for each of these $n$ we have

$$
\begin{aligned}
\log d_{\pi}^{(s)}(S\lceil n) & =s n+\sum_{i=0}^{n-1} \log \pi(w \uparrow i, w[i]) \\
& =s n-\mathcal{L}_{\pi}^{\log }(S\lceil n) \\
& \geq s n-t n \\
& =(s-t) n,
\end{aligned}
$$

so it follows that $S \in S^{\infty}\left[d_{\pi}^{(s)}\right]$ and $X \subseteq S^{\infty}\left[d_{\pi}^{(s)}\right]$. Therefore, $\operatorname{dim}_{\Delta}(X) \leq s$. As this holds for all rational $s>\operatorname{unpred}_{\Delta}^{\log }(X)$, we have $\operatorname{dim}_{\Delta}(X) \leq \operatorname{unpred}_{\Delta}^{\log }(X)$. The proof that $\operatorname{Dim}_{\Delta}(X) \leq$ $\operatorname{Unpred}_{\Delta}^{\log }(X)$ is analogous.

\subsubsection{Entropy Rates and Kolmogorov Complexity}

In this section we show that versions of the entropy rate and Kolmogorov complexity characterizations of constructive dimension (Theorems 3.25 and 3.14) also hold for the computable and polynomial-space dimensions.

In the following definition, comp is the class of all computable space bounds and poly is the class of all polynomials. We use $\mathrm{KS}^{f}$ to denote $f$-space-bounded Kolmogorov complexity.

Definition. For any $X \subseteq \mathbf{C}$, we define the quantities

$$
\begin{aligned}
\mathcal{K} \mathcal{S}_{\text {comp }}(X) & =\inf _{f \in \text { comp }} \sup _{S \in X} \liminf _{n \rightarrow \infty} \frac{\operatorname{KS}^{f}(S\lceil n)}{n}, \\
\mathcal{K} \mathcal{S}_{\text {comp }}^{\text {str }}(X) & =\inf _{f \in \operatorname{comp}} \sup _{S \in X} \limsup _{n \rightarrow \infty} \frac{\mathrm{KS}^{f}(S\lceil n)}{n}, \\
\mathcal{K} \mathcal{S}_{\text {poly }}(X) & =\inf _{p \in \text { poly }} \sup _{S \in X} \liminf _{n \rightarrow \infty} \frac{\mathrm{KS}^{p}(S\lceil n)}{n},
\end{aligned}
$$

and

$$
\mathcal{K} \mathcal{S}_{\text {poly }}^{\text {str }}(X)=\inf _{p \in \text { poly }} \sup _{S \in X} \limsup _{n \rightarrow \infty} \frac{\mathrm{KS}^{p}(S \uparrow n)}{n}
$$

The main result of this section gives new characterizations of the computable and polynomial- 
space dimensions. Here we use the $\mathcal{H}_{\mathcal{C}}(X)$ and $\mathcal{H}_{\mathcal{C}}^{\text {str }}(X)$ entropy rates from Section 3.2.2.

Theorem 3.34. For all $X \subseteq \mathbf{C}$,

$$
\begin{gathered}
\operatorname{dim}_{\text {comp }}(X)=\mathcal{H}_{\text {DEC }}(X)=\mathcal{K} \mathcal{S}_{\text {comp }}(X), \\
\operatorname{Dim}_{\text {comp }}(X)=\mathcal{H}_{\text {DEC }}^{\text {str }}(X)=\mathcal{K} \mathcal{S}_{\text {comp }}^{\text {str }}(X), \\
\operatorname{dim}_{\text {pspace }}(X)=\mathcal{H}_{\text {PSPACE }}(X)=\mathcal{K} \mathcal{S}_{\text {poly }}(X),
\end{gathered}
$$

and

$$
\operatorname{Dim}_{\text {pspace }}(X)=\mathcal{H}_{\mathrm{PSPACE}}^{\mathrm{str}}(X)=\mathcal{K} \mathcal{S}_{\text {poly }}^{\text {str }}(X)
$$

Theorem 3.34 will follow from the next few lemmas. First, we show that the dimensions are upper bounded by the entropy rates.

Lemma 3.35. Let $\Delta \in\{$ comp, pspace $\}$. For any $X \subseteq \mathbf{C}$,

$$
\operatorname{dim}_{\Delta}(X) \leq \mathcal{H}_{R(\Delta)}(X)
$$

and

$$
\operatorname{Dim}_{\Delta}(X) \leq \mathcal{H}_{R(\Delta)}^{\text {str }}(X)
$$

Proof. We first give the proof for $\Delta=$ comp. The case $\mathcal{H}_{\mathrm{DEC}}(X)=1$ is trivial, so assume $\mathcal{H}_{\mathrm{DEC}}(X)<1$ and let $1>r>\mathcal{H}_{\mathrm{DEC}}(X)$ be arbitrary. Take a decidable $A$ and $2^{s}, 2^{t}$ rational such that $X \subseteq A^{\text {i.o. }}$ and $H_{A}<s<t<r$. Recall the construction of a $t$-gale $d$ that succeeds on $A^{\text {i.o. }}$ from the proof of Lemma 3.23. For each $n \in \mathbb{N}$, we first defined $d_{n}:\{0,1\}^{*} \rightarrow[0, \infty)$ by

$$
d_{n}(w)= \begin{cases}2^{-t(n-|w|)} \cdot\left|\left\{v \in A_{=n} \mid w \sqsubseteq v\right\}\right| & \text { if }|w| \leq n \\ 2^{(t-1)(|w|-n)} d_{n}(w\lceil n) & \text { if }|w|>n .\end{cases}
$$


and then defined $d:\{0,1\}^{*} \rightarrow[0, \infty)$ by

$$
d=\sum_{n=0}^{\infty} 2^{(t-s) n} d_{n}
$$

We will show that $d$ is computable. For this, choose a natural number $k>\frac{1}{t-s}$. Define a function $\hat{d}:\{0,1\}^{*} \times \mathbb{N} \rightarrow[0, \infty) \cap \mathbb{Q}$ by

$$
\hat{d}(w, r)=\sum_{n=0}^{k r+|w|} 2^{(s-t) n} d_{n}(w) .
$$

Then $\hat{d}$ is exactly computable. For all $n, d_{n}(w) \leq 1$ for all $w$ with $|w| \geq n$, so for any precision $r \in \mathbb{N}$,

$$
\begin{aligned}
|d(w)-\hat{d}(w, r)| & =\sum_{n=k r+|w|+1}^{\infty} 2^{(s-t) n} d_{n}(w) \\
& \leq \sum_{n=k r+|w|+1}^{\infty} 2^{(s-t) n} \\
& \leq \sum_{n=k r+1}^{\infty} 2^{(s-t) n} \\
& =2^{(s-t)(k r)} \\
& <2^{-r}
\end{aligned}
$$

Therefore $\hat{d}$ demonstrates that $d$ is computable. Then $\operatorname{dim}_{\text {comp }}(X) \leq t<r$ because $X \subseteq$ $A^{\text {i.o. }} \subseteq S^{\infty}[d]$. It follows that $\operatorname{dim}_{\text {comp }}(X) \leq \mathcal{H}_{\mathrm{DEC}}(X)$ because $r>\mathcal{H}_{\mathrm{DEC}}(X)$ is arbitrary. The proof that $\operatorname{Dim}_{\text {comp }}(X) \leq \mathcal{H}_{\mathrm{DEC}}^{\text {str }}(X)$ is analogous.

For $\Delta=$ pspace, the same construction works. If $A \in \mathrm{PSPACE}$, then $d_{n}(w)$ can be exactly computed in space that is polynomial in $n+|w|$ by reusing space. Therefore $\hat{d}(w, r)$ is exactly computable in space polynomial in $r+|w|$, so $d$ is a polynomial-space computable $t$-gale.

We now show that the dimensions are lower bounded by the entropy rates. While it seems difficult to extend the upper bounds in Lemma 3.35 to the polynomial-time case, the lower 
bounds do hold for polynomial time.

Lemma 3.36. Let $r(\mathrm{comp})=$ DEC, $r$ (pspace $)=$ PSPACE, and $r(\mathrm{p})=\mathrm{P}$. For all $X \subseteq \mathbf{C}$ and $\Delta \in\{$ comp, pspace, $\mathrm{p}\}$,

$$
\mathcal{H}_{r(\Delta)}(X) \leq \operatorname{dim}_{\Delta}(X)
$$

and

$$
\mathcal{H}_{r(\Delta)}^{\mathrm{str}}(X) \leq \operatorname{Dim}_{\Delta}(X)
$$

Proof. Let $d$ be an exactly $\Delta$-computable s-gale with $X \subseteq S^{\infty}[d]$. Assume without loss of generality that $d(\lambda)<1$ and define $A=\{w \mid d(w)>1\}$. For all $n \in \mathbb{N}$, we have

$$
\sum_{w \in\{0,1\}^{n}} d(w) \leq 2^{s n}
$$

so $\left|A_{=n}\right| \leq 2^{s n}$. Also, $X \subseteq S^{\infty}[d] \subseteq A^{\text {i.o. }}$. For any $t>s$,

$$
\sum_{w \in A} 2^{-t|w|}=\sum_{n=0}^{\infty} 2^{-t n}\left|A_{=n}\right| \leq \sum_{n=0}^{\infty} 2^{(s-t) n}<\infty
$$

so $H_{A} \leq t$. Therefore $H_{A} \leq s$. Since $A \in r(\Delta)$, we have $\mathcal{H}_{r(\Delta)}(X) \leq s$. It follows that $\mathcal{H}_{r(\Delta)}(X) \leq \operatorname{dim}_{\Delta}(X)$. The proof that $\mathcal{H}_{r(\Delta)}^{\text {str }}(X) \leq \operatorname{Dim}_{\Delta}(X)$ is analogous.

The next lemma equates the entropy rates with the Kolmogorov complexity quantities defined at the beginning of this section.

Lemma 3.37. For all $X \subseteq \mathbf{C}$,

$$
\begin{gathered}
\mathcal{H}_{\text {DEC }}(X)=\mathcal{K} \mathcal{S}_{\text {comp }}(X), \\
\mathcal{H}_{\text {DEC }}^{\text {str }}(X)=\mathcal{K} \mathcal{S}_{\text {comp }}^{\text {str }}(X), \\
\mathcal{H}_{\text {PSPACE }}(X)=\mathcal{K} \mathcal{S}_{\text {poly }}(X), \\
\mathcal{H}_{\text {PSPACE }}^{\text {str }}(X)=\mathcal{K} \mathcal{S}_{\text {poly }}^{\text {str }}(X) .
\end{gathered}
$$


Proof. Let $t>s>\mathcal{K} \mathcal{S}_{\text {comp }}(X)$ be rational and let $f$ be a computable space bound such that

$$
\liminf _{n \rightarrow \infty} \frac{\mathrm{KS}^{f}(S \uparrow n)}{n}<s
$$

for all $S \in X$. Define

$$
A=\left\{w\left|\operatorname{KS}^{f}(w) \leq s\right| w \mid\right\}
$$

Then $A \in \mathrm{DEC}$ and $X \subseteq A^{\text {i.o. }}$. Also, $\left|A_{=n}\right| \leq 2^{\lceil s n\rceil} \leq 2^{t n}$ for all $n$, so $H_{A} \leq t$. Therefore $\mathcal{H}_{\text {DEC }}(X) \leq t$. As $t>\mathcal{K} \mathcal{S}_{\text {comp }}(X)$ is an arbitrary rational, we have $\mathcal{H}_{\text {DEC }}(X) \leq \mathcal{K} \mathcal{S}_{\text {comp }}(X)$. The inequality $\mathcal{H}_{\mathrm{DEC}}^{\mathrm{str}}(X) \leq \mathcal{K} \mathcal{S}_{\text {comp }}^{\mathrm{str}}(X)$ is established analogously. The corresponding inequalities for the polynomial-space part follow in the same way using the fact that $\{w \mid$ $\left.\mathrm{KS}^{f}(w)<s|w|\right\}$ is in PSPACE if $f$ is a polynomial.

Let $s>\mathcal{H}_{\mathrm{DEC}}(X)$, and let $A \in \mathrm{DEC}$ such that $H_{A}<s$ and $X \subseteq A^{\text {i.o. }}$. Let $f(n) \geq n$ be a computable space bound in which $A$ is decidable. Any $w \in A_{=n}$ can be described by giving $n$ and its index within a listing of $A_{=n}$ in lexicographic order. By reusing space, $w$ can be computed from this description in $O(f(n))$ space. Therefore

$$
\mathrm{KS}^{O(f)}(w) \leq \log \left|A_{=n}\right|+O(\log n)
$$

Let $S \in X$. Then

$$
\left(\exists^{\infty} n\right) S \uparrow n \in A_{=n}
$$

Also, since $H_{A}<s$,

$$
\left(\forall^{\infty} n\right) \frac{\log \left|A_{=n}\right|}{n}<s
$$

so

$$
\left(\exists^{\infty} n\right) \frac{\mathrm{KS}^{O(f)}(S\lceil n)}{n} \leq \frac{\log \left|A_{=n}\right|+O(\log n)}{n}<s+o(1) .
$$

Therefore

$$
\liminf _{n \rightarrow \infty} \frac{\mathrm{KS}^{O(f)}(S\lceil n)}{n} \leq s
$$


Because $s>H_{A}$ is arbitrary, this establishes that $\mathcal{K} \mathcal{S}_{\text {comp }}(X) \leq \mathcal{H}_{\mathrm{DEC}}(X)$. The proofs of the remaining three inequalities are analogous.

Theorem 3.34 now follows immediately from Lemmas 3.35, 3.36, and 3.37.

\subsection{Scaled Dimension}

To overcome limitations of resource-bounded dimension for investigating complexity classes within ESPACE, Hitchcock, Lutz, and Mayordomo [23] introduced for each integer $i \in \mathbb{Z}$ the $i^{\text {th }}$-order scaled dimension $\operatorname{dim}_{\Delta}^{(i)}(X)$ and also the $i^{\text {th }}$-order scaled dimension in $R(\Delta)$ $\operatorname{dim}^{(i)}(X \mid R(\Delta))=\operatorname{dim}_{\Delta}^{(i)}(X \cap R(\Delta))$. For any class $X$ and $i \in \mathbb{Z}, \operatorname{dim}_{\Delta}^{(i)}(X) \in[0,1]$, and if it is less than 1 , then $\mu_{\Delta}(X)=0$. The quantity $\operatorname{dim}_{\Delta}^{(i)}(X)$ is nondecreasing in $i$, and there is at most one $i \in \mathbb{Z}$ for which $0<\operatorname{dim}_{\Delta}^{(i)}(X)<1$. The $0^{\text {th }}$-order dimension, $\operatorname{dim}_{\Delta}^{(0)}(\cdot)$, is precisely the standard unscaled dimension, and the other orders can be more useful than it for certain complexity classes. To illustrate this, we mention some examples from circuit-size complexity. For a function $s: \mathbb{N} \rightarrow \mathbb{N}$, let $\operatorname{SIZE}(s(n))$ consist of all languages decidable by nonuniform Boolean circuit families of size $s(n)$. Lutz [30] showed that

$$
\operatorname{dim}\left(\operatorname{SIZE}\left(\alpha \frac{2^{n}}{n}\right) \mid \operatorname{ESPACE}\right)=\alpha
$$

for all $\alpha \in(0,1)$. Circuit size bounds of the from $2^{\alpha n}$ and $2^{n^{\alpha}}$ are typically of more interest in complexity theory, but (3.1) implies that $\operatorname{SIZE}\left(2^{\alpha n}\right)$ and $\operatorname{SIZE}\left(2^{n^{\alpha}}\right)$ have dimension 0 in $\mathrm{E}$ for all $\alpha \in(0,1)$. For these size bounds, the scaled dimensions are useful; in [23] it is shown that

$$
\operatorname{dim}^{(1)}\left(\operatorname{SIZE}\left(2^{\alpha n}\right) \mid \operatorname{ESPACE}\right)=\alpha
$$

and

$$
\operatorname{dim}^{(2)}\left(\operatorname{SIZE}\left(2^{n^{\alpha}}\right) \mid \operatorname{ESPACE}\right)=\alpha
$$

for any $\alpha \in(0,1)$.

In this section we review the essentials of resource-bounded scaled dimension and develop 
some new tools for working with it. The principle concept is a scale, which is a function $g: H \times[0, \infty) \rightarrow \mathbb{R}$, where $H=(a, \infty)$ for some $a \in \mathbb{R} \cup\{-\infty\}$.

Definition. A scale is a continuous function $g: H \times[0, \infty) \longrightarrow \mathbb{R}$ with the following properties.

1. $H=(a, \infty)$ for some $a \in \mathbb{R} \cup\{-\infty\}$.

2. $g(m, 1)=m$ for all $m \in H$.

3. $g(m, 0)=g\left(m^{\prime}, 0\right) \geq 0$ for all $m, m^{\prime} \in H$.

4. For every sufficiently large $m \in H$, the function $s \mapsto g(m, s)$ is nonnegative and strictly increasing.

5. For all $s^{\prime}>s \geq 0, \lim _{m \rightarrow \infty}\left[g\left(m, s^{\prime}\right)-g(m, s)\right]=\infty$.

The canonical example of a scale is the function $g_{0}: \mathbb{R} \times[0, \infty) \rightarrow \mathbb{R}$ defined by $g_{0}(m, s)=$ sm. This scale is used in the standard (unscaled) dimension. Other scales of interest are obtained from $g_{0}$ by rescaling and reflection operations.

Definition. Let $g: H \times[0, \infty) \rightarrow \mathbb{R}$ be a scale.

1. The first rescaling of $g$ is the scale $g^{\#}: H^{\#} \times[0, \infty) \longrightarrow \mathbb{R}$ defined by

$$
\begin{gathered}
H^{\#}=\left\{2^{m} \mid m \in H\right\} \\
g^{\#}(m, s)=2^{g(\log m, s)} .
\end{gathered}
$$

2. The reflection of $g$ is the scale $g^{R}: H \times[0, \infty) \rightarrow \mathbb{R}$ defined by

$$
g^{R}(m, s)= \begin{cases}m+g(m, 0)-g(m, 1-s) & \text { if } 0 \leq s \leq 1 \\ g(m, s) & \text { if } s \geq 1\end{cases}
$$


If $g$ is a scale, then for all $m \in H^{\#}$ and $s \in[0, \infty)$,

$$
\log g^{\#}(m, s)=g(\log m, s)
$$

which means that a log-log graph of the function $m \mapsto g^{\#}(m, s)$ is precisely the ordinary graph of the function $m \mapsto g(m, s)$. This is the sense in which $g^{\#}$ is a rescaling of $g$.

A family of scales, one for each integer, is defined as follows.

Definition. 1. For each $i \in \mathbb{N}$, define $a_{i}$ by the recurrence $a_{0}=-\infty, a_{i+1}=2^{a_{i}}$.

2. For each $i \in \mathbb{Z}$, define the $i^{\text {th }}$ scale $g_{i}:\left(a_{|i|}, \infty\right) \times[0, \infty) \rightarrow \mathbb{R}$ by the following recursion.

(a) $g_{0}(m, s)=s m$.

(b) For $i \geq 0, g_{i+1}=g_{i}^{\#}$.

(c) For $i<0, g_{i}=g_{-i}^{R}$.

For clarity, we compute the first few scales. For all $s \in[0,1]$, if $m>a_{|i|}$, then $g_{i}(m, s)$ is defined by

$$
\begin{aligned}
g_{3}(m, s) & =2^{2^{(\log \log m)^{s}}} \\
g_{2}(m, s) & =2^{(\log m)^{s}} \\
g_{1}(m, s) & =m^{s} \\
g_{0}(m, s) & =s m \\
g_{-1}(m, s) & =m+1-m^{1-s} \\
g_{-2}(m, s) & =m+2-2^{(\log m)^{1-s}} \\
g_{-3}(m, s) & =m+4-2^{2^{(\log \log m)^{1-s}}} .
\end{aligned}
$$

Scaled dimension is defined using functions called scaled gales.

Definition. Let $i \in \mathbb{Z}$ and let $s \in[0, \infty)$. An $i^{\text {th }}$-order scaled $s$-gale (briefly, an $s^{(i)}$-gale) is 
a function $d:\{0,1\}^{>a_{|i|}} \rightarrow[0, \infty)$ such that for all $w \in\{0,1\}^{*}$ with $|w|>a_{|i|}$,

$$
d(w)=2^{-\Delta g_{i}(|w|, s)}[d(w 0)+d(w 1)]
$$

where $\Delta g_{i}:\left(a_{|i|}, \infty\right) \times[0, \infty) \rightarrow \mathbb{R}$ is defined by

$$
\Delta g_{i}(m, s)=g_{i}(m+1, s)-g_{i}(m, s)
$$

Note that an $s^{(0)}$-gale is simply an $s$-gale.

Success sets of scaled gales are used to define scaled dimension.

Definition. Let $X \subseteq \mathbf{C}$ and $i \in \mathbb{Z}$.

1. The $i^{\text {th }}$-order scaled $\Delta$-dimension of $X$ is

$$
\operatorname{dim}_{\Delta}^{(i)}(X)=\inf \left\{\begin{array}{l|l}
s \mid \begin{array}{l}
\text { there exists a } \Delta \text {-computable } \\
s^{(i)} \text {-scaled gale d for which } X \subseteq S^{\infty}[d]
\end{array}
\end{array}\right\}
$$

2. The $i^{\text {th }}$-order scaled dimension of $X$ within $R(\Delta)$ is

$$
\operatorname{dim}^{(i)}(X \mid R(\Delta))=\operatorname{dim}_{\Delta}^{(i)}(X \cap R(\Delta))
$$

The $0^{\text {th }}$-order dimension $\operatorname{dim}_{\Delta}^{(0)}(\cdot)$ is precisely the dimension $\operatorname{dim}_{\Delta}(\cdot)$ defined in Section 3.3 and the other orders are interpreted as rescalings of this concept.

The following lemma relates resource-bounded scaled dimension to resource-bounded measure.

Lemma 3.38. ([23]) For any class $X \subseteq \mathbf{C}$ and $i \in \mathbb{Z}$,

$$
\operatorname{dim}_{\Delta}^{(i)}(X)<1 \Rightarrow \mu_{\Delta}(X)=0
$$


and

$$
\operatorname{dim}^{(i)}(X \mid R(\Delta))<1 \Rightarrow \mu(X \mid R(\Delta))=0
$$

The following is another key property of scaled dimension.

Theorem 3.39. ([23]) Let $X \subseteq \mathbf{C}$ and $i \in \mathbb{Z}$. If $\operatorname{dim}_{\Delta}^{(i+1)}(X)<1$, then $\operatorname{dim}_{\Delta}^{(i)}(X)=0$.

This theorem tells us that for every class $X$, the sequence of $\operatorname{dimensions} \operatorname{dim}_{\Delta}^{(i)}(X)$ for $i \in \mathbb{Z}$ satisfies exactly one of the following three conditions.

(i) $\operatorname{dim}_{\Delta}^{(i)}(X)=0$ for all $i \in \mathbb{Z}$.

(ii) $\operatorname{dim}_{\Delta}^{(i)}(X)=1$ for all $i \in \mathbb{Z}$.

(iii) There exist $i^{*} \in \mathbb{Z}$ such that $\operatorname{dim}_{\Delta}^{(i)}(X)=0$ for all $i<i^{*}$ and $\operatorname{dim}_{\Delta}^{(i)}(X)=1$ for all $i>i^{*}$.

\subsubsection{Unpredictability Tools}

This section provides some tools involving measures and the log-loss concept that are useful for working with the scaled dimensions. In Section 3.3.1 we showed that log-loss unpredictability is equivalent to dimension. We similarly characterize scaled dimension using the log-loss of measures.

Definition. A measure on $\mathbf{C}$ is a function $\rho:\{0,1\}^{*} \rightarrow[0, \infty)$ satisfying

$$
\rho(w)=\rho(w 0)+\rho(w 1)
$$

for all $w \in\{0,1\}^{*}$.

Measures have the following fundamental relationship with scaled gales. This extends Schnorr's "likelihood ratio" characterization of martingales [55].

Observation 3.40. Let $i \in \mathbb{Z}$ and $s \in[0, \infty)$.

1. If $\rho:\{0,1\}^{*} \rightarrow[0, \infty)$ is a measure, then the function $d_{\rho}:\{0,1\}^{>a_{|i|}} \rightarrow[0, \infty)$ defined by

$$
d_{\rho}(w)=2^{g_{i}(|w|, s)} \rho(w)
$$


for all $w \in\{0,1\}^{>a_{|i|}}$ is an $s^{(i)}$-gale.

2. If $d:\{0,1\}^{>a_{|i|}} \rightarrow[0, \infty)$ is an $s^{(i)}$-gale, then the function $\rho_{d}:\{0,1\}^{*} \rightarrow[0, \infty)$ defined by

$$
\rho_{d}(w)=2^{-g_{i}(|w|, s)} d(w)
$$

for all $w \in\{0,1\}^{>a_{|i|}}$ and

$$
\rho_{d}(w)=\sum_{|v|=a_{|i|}+1-|w|} \rho_{d}(w v)
$$

for all $w \in\{0,1\} \leq a_{|i|}$ is a measure.

The following lemma relates the scaled dimension of a class to limits involving scales and logarithms of measures.

Lemma 3.41. Let $X \subseteq \mathbf{C}$ and let $i \in \mathbb{Z}$.

1. If $s>\operatorname{dim}_{\Delta}^{(i)}(X)$, then there is a $\Delta$-computable measure $\rho$ such that

$$
\limsup _{n \rightarrow \infty} g_{i}(n, s)+\log \rho(A\lceil n)=\infty
$$

for all $A \in X$.

2. If $s<\operatorname{dim}_{\Delta}^{(i)}(X)$, then for any $\Delta$-computable measure $\rho$ there is an $A_{\rho} \in X$ such that

$$
\lim _{n \rightarrow \infty} g_{i}(n, s)+\log \rho\left(A_{\rho}\lceil n)=-\infty\right.
$$

Proof. Let $r$ be rational with $s>r>\operatorname{dim}_{\Delta}^{(i)}(X)$ and let $d$ be a $\Delta$-computable $r^{(i)}$-gale succeeding on $X$. Then the measure $\rho_{d}$ from Observation 3.40 is also $\Delta$-computable. Let $A \in X$. There are infinitely many $n \in \mathbb{N}$ such that $d\left(A\lceil n) \geq 1\right.$ since $A \in S^{\infty}[d]$. For such $n$,

$$
\begin{aligned}
g_{i}(n, s)+\log \rho_{d}(A\lceil n) & =g_{i}(n, s)-g_{i}(n, r)+\log d(A\lceil n) \\
& \geq g_{i}(n, s)-g_{i}(n, r) .
\end{aligned}
$$


Part 1 follows because $r<s$.

For part 2 , let $\rho$ be a $\Delta$-computable measure. Let $t$ be rational with $s<t<\operatorname{dim}_{\Delta}^{(i)}(X)$ and obtain the $t^{(i)}$-gale $d_{\rho}$ from Observation 3.40. Then $X \nsubseteq S^{\infty}\left[d_{\rho}\right]$ because $d_{\rho}$ is $\Delta$-computable, so there is an $A_{\rho} \in X$ and a constant $c$ such that $d\left(A\lceil n) \leq c\right.$ for all $n>a_{|i|}$. Then

$$
\begin{aligned}
g_{i}(n, s)+\log \rho(A\lceil n) & =g_{i}(n, s)-g_{i}(n, t)+\log d_{\rho}(A\lceil n) \\
& \leq g_{i}(n, s)-g_{i}(n, t)+\log c
\end{aligned}
$$

so the claim follows because $s<t$.

Lemma 3.41 asserts that if the $i^{\text {th }}$-order scaled dimension of a class $X$ is less than $s$ then there is a measure $\rho$ such that for every $A \in X$, there are prefixes $w \sqsubseteq A$ where the log-loss quantity

$$
-\log \rho(w)
$$

is arbitrarily less than $g_{i}(|w|, s)$.

It is often convenient to replace computable measures by exactly computable measures.

Lemma 3.42. Let $\rho$ be a measure that is computable in $t(n)$ time (respectively, space). Then there is a measure $\tilde{\rho}$ that is exactly computable in $O(n \cdot t(3 n))$ time (respectively, space) such that

$$
\log \tilde{\rho}(w) \geq \log \rho(w)-c
$$

for all $w \in\{0,1\}^{*}$ where $c$ is a constant that is independent of $w$.

Proof. We assume that $\rho(w) \geq 2^{-|w|}$ for all $w \in\{0,1\}^{*}$. (If $\rho$ does not satisfy this condition, add $2^{-|w|}$ to $\rho(w)$ to obtain a measure that does and use it instead.)

Let $\hat{\rho}: \mathbb{N} \times\{0,1\}^{*} \rightarrow[0, \infty)$ be an approximation of $\rho$. For all $w \in\{0,1\}^{*}$, define

$$
\rho^{\prime}(w)=\hat{\rho}(2|w|, w)
$$


The measure $\tilde{\rho}:\{0,1\}^{*} \rightarrow[0, \infty)$ is defined by

$$
\tilde{\rho}(\lambda)=\rho^{\prime}(0)+\rho^{\prime}(1)
$$

and

$$
\tilde{\rho}(w b)=\frac{\rho^{\prime}(w b)}{\rho^{\prime}(w 0)+\rho^{\prime}(w 1)} \tilde{\rho}(w)
$$

for all $w \in\{0,1\}^{*}$ and $b \in\{0,1\}$. If $\hat{\rho}$ is exactly computable in $t(n)$ time, then $\rho^{\prime}(w)$ is computable in $t(2|w|+|w|)$ time, so we can exactly compute $\tilde{\rho}(w)$ in $O(|w| \cdot t(3|w|))$ time. Similarly, if $\hat{\rho}$ is computable in $t(n)$ space, then $\tilde{\rho}$ is computable in $O(n \cdot t(3 n))$ space.

For any $w \in\{0,1\}^{+}$, we have

$$
\begin{aligned}
\frac{\rho^{\prime}(w)}{\rho^{\prime}(w 0)+\rho^{\prime}(w 1)} & \geq \frac{\rho(w)-2^{-2|w|}}{\rho(w 0)+2^{-2(|w|+1)}+\rho(w 1)+2^{-2(|w|+1)}} \\
& =\frac{\rho(w)-2^{-2|w|}}{\rho(w)+2^{-2|w|-1}} \\
& \geq \frac{2^{-|w|}-2^{-2|w|}}{2^{-|w|}+2^{-2|w|-1}} \\
& =\frac{2^{|w|}-1}{2^{|w|}+\frac{1}{2}} \\
& =1-\frac{\frac{3}{2}}{2^{|w|}+\frac{1}{2}} \\
& \geq 1-\frac{2}{2^{|w|+1}} \\
& =1-2^{-|w|}
\end{aligned}
$$

with the second inequality holding because

$$
\frac{\alpha-\epsilon}{\alpha+\frac{\epsilon}{2}}
$$


is an increasing function of $\alpha$ and $2^{-|w|}$ is the minimum possible value for $\rho(w)$. Therefore

$$
\begin{aligned}
\log \tilde{\rho}(w) & =\log \left(\prod_{i=1}^{|w|} \frac{\rho^{\prime}(w\lceil i)}{\rho^{\prime}\left((w\lceil i-1) 0)+\rho^{\prime}((w\lceil i-1) 1)\right.}\right) \tilde{\rho}(\lambda) \\
& =\log \rho^{\prime}(w)+\sum_{i=1}^{|w|-1} \log \frac{\rho^{\prime}(w\lceil i)}{\rho^{\prime}\left((w\lceil i) 0)+\rho^{\prime}((w\lceil i) 1)\right.}+\log \frac{\rho^{\prime}(0)+\rho^{\prime}(1)}{\rho^{\prime}(0)+\rho^{\prime}(1)} \\
& \geq \log \rho^{\prime}(w)+\sum_{i=1}^{|w|-1} \log 1-2^{-i} \\
& \geq \log \rho^{\prime}(w)+\sum_{i=1}^{|w|-1} \frac{-2^{-i}}{\ln 2} \\
& \geq \log \rho^{\prime}(w)-\frac{1}{\ln 2} .
\end{aligned}
$$

Also, again using the fact that $\rho(w) \geq 2^{-|w|}$,

$$
\begin{aligned}
\log \rho^{\prime}(w) & \geq \log \left[\rho(w)-2^{-2|w|}\right] \\
& \geq \log \rho(w) \frac{2^{-|w|}-2^{-2|w|}}{2^{-|w|}} \\
& =\log \rho(w)\left(1-2^{-|w|}\right) \\
& \geq \log \rho(w)-\frac{2^{-|w|}}{\ln 2} .
\end{aligned}
$$

Combining the above, we have

$$
\log \tilde{\rho}(w) \geq \log \rho(w)-\frac{2}{\ln 2}
$$

for all $w \in\{0,1\}^{+}$. The lemma holds with $c=\left\lceil\max \left\{\frac{2}{\ln 2}, \log \frac{\rho(\lambda)}{\tilde{\rho}(\lambda)}\right\}\right\rceil$.

The measures that are exactly computable within a fixed time or space bound are uniformly exactly computable with slightly more time or space.

Lemma 3.43. For any time constructible functions $t(n)$ and $t^{\prime}(n)$ with $t(n) \log t(n)=o\left(t^{\prime}(n)\right)$, the family of exactly $t(n)$-time computable measures is uniformly exactly computable in $t^{\prime}(n)$ time. If $t(n)=o\left(t^{\prime}(n)\right)$, then the family of exactly $t(n)$-space computable measures is uniformly 
exactly computable in $t^{\prime}(n)$-space.

Proof. There is a uniform enumeration $\left(M_{i} \mid i \in \mathbb{N}\right)$ of all $t(n)$-time (respectively, $t(n)$-space) clocked Turing machines such that for all $i \in \mathbb{N}, M_{i}(w)$ can be computed in $O(t(|w|) \log t(|w|))$ time (respectively, $O(t(|w|))$ space) for all $w \in\{0,1\}^{*}$. (Here the constants in the $O(\cdot)$ depend on $i$ but not on $|w|$.$) Define \rho_{i}:\{0,1\}^{*} \rightarrow[0, \infty)$ inductively by $\rho_{i}(\lambda)=M_{i}(\lambda)$ and

$$
\begin{gathered}
\rho_{i}(w 0)= \begin{cases}M_{i}(w 0) & \text { if } M_{i}(w 0) \leq \rho_{i}(w) \\
M_{i}(w) & \text { otherwise }\end{cases} \\
\rho_{i}(w 1)=\rho_{i}(w)-\rho_{i}(w 0)
\end{gathered}
$$

for all $w \in\{0,1\}^{*}$. Then each $\rho_{i}$ is a measure, and the family is uniformly computable in $t^{\prime}(n)$ time (respectively, $t^{\prime}(n)$ space). Also, if $\rho$ is a measure that is exactly computed by $M_{i}$ in $t(n)$ time, then $\rho_{i}(w)=\rho(w)$ for all $w$.

Uniformly exactly computable families of measures can be combined into a single measure in an efficient manner.

Lemma 3.44. Let $\left(\rho_{k} \mid k \in \mathbb{N}\right)$ be a uniformly exactly $\Delta$-computable family of measures. There is a $\Delta$-computable measure $\rho^{*}$ such that for any $k$, there is a constant $c_{k}$ such that

$$
\log \rho^{*}(w) \geq \log \rho_{k}(w)-c_{k}
$$

for all $w \in\{0,1\}^{*}$.

Proof. Define

$$
\rho^{*}(w)=\sum_{k=0}^{\infty} \frac{\rho_{k}(w)}{2^{k} \rho_{k}(\lambda)} .
$$

Then $\rho$ is a measure by linearity. Also, $\rho^{*}$ is $\Delta$-computable by the approximation function $\hat{\rho}^{*}: \mathbb{N} \times\{0,1\}^{*} \rightarrow[0, \infty)$ defined by

$$
\hat{\rho}^{*}(r, w)=\sum_{k=0}^{r} \frac{\rho_{k}(w)}{2^{k} \rho_{k}(\lambda)}
$$


since

$$
\begin{aligned}
\left|\rho^{*}(w)-\hat{\rho}^{*}(r, w)\right| & =\sum_{k=r+1}^{\infty} \frac{\rho_{k}(w)}{2^{k} \rho_{k}(\lambda)} \\
& \leq \sum_{k=r+1}^{\infty} \frac{\rho_{k}(\lambda)}{2^{k} \rho_{k}(\lambda)} \\
& =2^{-r} .
\end{aligned}
$$

Let $k \in \mathbb{N}$. For any $w \in\{0,1\}^{*}$,

$$
\begin{aligned}
\log \rho^{*}(w) & \geq \log \frac{\rho_{k}(w)}{2^{k} \rho_{k}(\lambda)} \\
& =\log \rho_{k}(w)-k-\rho_{k}(\lambda),
\end{aligned}
$$

so the lemma holds with $c_{k}=k+\rho_{k}(\lambda)$.

We now combine the preceding lemmas to obtain a tool that will be useful in calculating scaled dimensions.

Theorem 3.45. Let $X \subseteq \mathbf{C}, i \in \mathbb{Z}$, and $k \in \mathbb{N}$.

1. If for each $A \in X$ there is a measure $\rho_{A}$ computable in $O\left(n^{k}\right)$ time such that

$$
\left(\exists c_{A} \in \mathbb{Z}\right)\left(\exists^{\infty} n\right) g_{i}(n, s)+\log \rho_{A}\left(A\lceil n) \geq c_{A}\right.
$$

then $\operatorname{dim}_{\mathrm{p}}^{(i)}(X) \leq s$.

2. If for each $A \in X$ there is a measure $\rho_{A}$ computable in $O\left(2^{\log n^{k}}\right)$ time such that (3.3) holds, then then $\operatorname{dim}_{\mathrm{P}_{2}}^{(i)}(X) \leq s$.

3. If for each $A \in X$ there is a measure $\rho_{A}$ computable in $O\left(n^{k}\right)$ space such that (3.3) holds, then then $\operatorname{dim}_{\text {pspace }}^{(i)}(X) \leq s$.

4. If for each $A \in X$ there is a measure $\rho_{A}$ computable in $O\left(2^{\log n^{k}}\right)$ space such that (3.3) holds, then then $\operatorname{dim}_{\mathrm{P}_{2} \text { space }}^{(i)}(X) \leq s$. 
Proof. From Lemmas 3.42, 3.43, and 3.44 we obtain an exactly $\Delta$-computable measure $\rho$ such that $\log \rho(w) \geq \log \rho_{A}(w)-b_{A}$ for all $w \in\{0,1\}^{*}$ where $b_{A}$ is a constant that depends on $A$ but not on $w$.

Let $t>s$. For any $A \in X$,

$$
g_{i}(n, t)+\log \rho\left(A\lceil n) \geq g_{i}(n, t)-g_{i}(n, s)+c_{A}-b_{A}\right.
$$

for infinitely many $n$. Therefore

$$
\limsup _{n \rightarrow \infty} g_{i}(n, t)+\log \rho(A\lceil n)=\infty
$$

since $t>s$. It follows from the contrapositive of Lemma $3.41(2)$ that $\operatorname{dim}_{\Delta}(X) \leq t$. 


\section{CONSTRUCTIVE DIMENSION AND THE ARITHMETICAL HIERARCHY}

In this chapter we use the arithmetical hierarchy (the effective Borel hierarchy) to investigate constructive dimension. In Section 4.2 we will prove a correspondence principle asserting that $\operatorname{dim}_{\mathrm{H}}(X)=\operatorname{cdim}(X)$ for any class $X$ that is a union of $\Pi_{1}^{0}$-sets. We investigate the arithmetical complexity of the classes $\mathrm{DIM}^{\alpha}$ and $\mathrm{DIM}_{\text {str }}^{\alpha}$ consisting of all sequences of dimension $\alpha$ and strong dimension $\alpha$, respectively, in Section 4.3. To classify $\operatorname{DIM}_{\text {str }}^{\alpha}$, we introduce a stronger effective Borel hierarchy. This new hierarchy is defined in Section 4.1 where the Borel and arithmetical hierarchies are also reviewed.

\subsection{Effective Borel Hierarchies}

We use $\boldsymbol{\Sigma}_{\mathbf{n}}^{\mathbf{0}}$ and $\boldsymbol{\Pi}_{\mathbf{n}}^{\mathbf{0}}$ to denote the levels of the Borel hierarchy for subsets of Cantor space. The levels of the arithmetical hierarchy (the corresponding effective hierarchy) are denoted by $\Sigma_{n}^{0}$ and $\Pi_{n}^{0}$.

We will also make use of the following more general hierarchy definition.

Definition. Let $\mathcal{P}$ be a class of predicates, let $n \geq 1$, and let $\mathcal{X} \subseteq \mathbf{C}$.

- $\mathcal{X} \in \Sigma_{n}^{0}[\mathcal{P}]$ if for some predicate $P \in \mathcal{P}$,

$$
A \in \mathcal{X} \Longleftrightarrow\left(\exists k_{n}\right)\left(\forall k_{n-1}\right) \cdots\left(Q k_{1}\right) P\left(k_{n}, \ldots, k_{2}, A \uparrow k_{1}\right),
$$

where $Q=\exists$ if $n$ is odd and $Q=\forall$ if $n$ is even. 
- $\mathcal{X} \in \Pi_{n}^{0}[\mathcal{P}]$ if for some predicate $P \in \mathcal{P}$,

$$
A \in \mathcal{X} \Longleftrightarrow\left(\forall k_{n}\right)\left(\exists k_{n-1}\right) \cdots\left(Q k_{1}\right) P\left(k_{n}, \ldots, k_{2}, A\left\lceil k_{1}\right),\right.
$$

where $Q=\forall$ if $n$ is odd and $Q=\exists$ if $n$ is even.

If we take $\mathcal{P}$ to be $\Delta_{1}^{0}$ (decidable predicates), then the above definition is equivalent to the standard arithmetical hierarchy; that is,

$$
\Sigma_{n}^{0}=\Sigma_{n}^{0}\left[\Delta_{1}^{0}\right]
$$

and

$$
\Pi_{n}^{0}=\Pi_{n}^{0}\left[\Delta_{1}^{0}\right]
$$

hold for all $n$. Also, if ALL is the class of all predicates, then we obtain the classical Borel hierarchy:

$$
\Sigma_{\mathbf{n}}^{\mathbf{0}}=\Sigma_{n}^{0}[\mathrm{ALL}]
$$

and

$$
\boldsymbol{\Pi}_{\mathbf{n}}^{\mathbf{0}}=\Pi_{n}^{0}[\mathrm{ALL}]
$$

In this chapter, we will also be interested in the cases where $\mathcal{P}$ is $\Sigma_{1}^{0}$ (enumerable predicates) or $\Pi_{1}^{0}$ (co-enumerable predicates). In some cases, the classes in the generalized hierarchy using these sets of predicates are no different that the standard arithmetical hierarchy classes. If $n$ is odd, then $\Sigma_{n}^{0}=\Sigma_{n}^{0}\left[\Sigma_{1}^{0}\right]$ as the existential quantifier in the $\Sigma_{1}^{0}$ predicate can be absorbed into the last quantifier in the definition of $\Sigma_{n}^{0}\left[\Delta_{1}^{0}\right]=\Sigma_{n}^{0}$. Analogously, $\Pi_{n}^{0}=\Pi_{n}^{0}\left[\Pi_{1}^{0}\right]$ for odd $n$, and for even $n$ we have $\Sigma_{n}^{0}=\Sigma_{n}^{0}\left[\Pi_{1}^{0}\right]$ and $\Pi_{n}^{0}=\Pi_{n}^{0}\left[\Sigma_{1}^{0}\right]$. On the other hand, using the complementary set of predicates defines an effective hierarchy that is distinct from and interleaved with the arithmetical hierarchy. 
Proposition 4.1. 1. If $n$ is odd, then

$$
\Sigma_{n}^{0} \subsetneq \Sigma_{n}^{0}\left[\Pi_{1}^{0}\right] \subsetneq \Sigma_{n+1}^{0}
$$

and

$$
\Pi_{n}^{0} \subsetneq \Pi_{n}^{0}\left[\Sigma_{1}^{0}\right] \subsetneq \Pi_{n+1}^{0}
$$

2. If $n$ is even, then

$$
\Sigma_{n}^{0} \subsetneq \Sigma_{n}^{0}\left[\Sigma_{1}^{0}\right] \subsetneq \Sigma_{n+1}^{0}
$$

and

$$
\Pi_{n}^{0} \subsetneq \Pi_{n}^{0}\left[\Pi_{1}^{0}\right] \subsetneq \Pi_{n+1}^{0}
$$

Proof. We only show $\Sigma_{n}^{0} \subsetneq \Sigma_{n}^{0}\left[\Pi_{1}^{0}\right] \subsetneq \Sigma_{n+1}^{0}$ for odd $n$; the arguments for the other statements are analogous.

The inclusion $\Sigma_{n}^{0} \subseteq \Sigma_{n}^{0}\left[\Pi_{1}^{0}\right]$ is obvious. To show that it is proper, let $P$ be a predicate that is complete for the class of $\Pi_{n}^{0}$ predicates. Then there is a decidable predicate $R$ such that

$$
P(n) \Longleftrightarrow\left(\forall k_{n}\right)\left(\exists k_{n-1}\right) \cdots\left(\forall k_{1}\right) R\left(n, k_{n}, \cdots, k_{1}\right) .
$$

Define $\mathcal{X} \subseteq \mathbf{C}$ as

$$
\mathcal{X}=\bigcup_{n \in P} 0^{n} 1 \mathbf{C}
$$

Then $\mathcal{X} \in \Sigma_{n}^{0}\left[\Pi_{1}^{0}\right]$ as we have

$$
\begin{aligned}
S \in \mathcal{X} & \Longleftrightarrow(\exists n) P(n) \text { and } 0^{n} 1 \sqsubseteq S \\
& \Longleftrightarrow(\exists n)\left(\forall k_{n}\right)\left(\exists k_{n-1}\right) \cdots\left(\forall k_{1}\right) R\left(n, k_{n}, \cdots, k_{1}\right) \text { and } 0^{n} 1 \sqsubseteq S \\
& \Longleftrightarrow \quad(\exists n)\left(\forall k_{n}\right)\left(\exists k_{n-1}\right) \cdots\left(\exists k_{2}\right) T\left(n, k_{n}, \cdots, k_{3}, S \uparrow k_{2}\right),
\end{aligned}
$$


where $T$ is the $\Pi_{1}^{0}$ predicate defined by

$$
T\left(n, k_{n}, \cdots, k_{3}, w\right) \Longleftrightarrow\left(\forall k_{1}\right) R\left(n, k_{n}, \cdots, k_{3},|w|, k_{1}\right) \text { and } 0^{n} 1 \sqsubseteq w \text {. }
$$

Now suppose that $\mathcal{X} \in \Sigma_{n}^{0}$. Then for some decidable predicate $U$,

$$
S \in X \Longleftrightarrow\left(\exists k_{n}\right)\left(\forall k_{n-1}\right) \cdots\left(\exists k_{1}\right) U\left(k_{n}, \cdots, k_{2}, S \uparrow k_{1}\right)
$$

We then have

$$
\begin{aligned}
n \in P & \Longleftrightarrow 0^{n} 1 \mathbf{C} \subseteq \mathcal{X} \\
& \Longleftrightarrow 0^{n} 10^{\infty} \in \mathcal{X} \\
& \Longleftrightarrow\left(\exists k_{n}\right)\left(\forall k_{n-1}\right) \cdots\left(\exists k_{1}\right) U\left(k_{n}, \cdots, k_{2}, 0^{n} 10^{\infty} \uparrow k_{1}\right),
\end{aligned}
$$

so $P$ is a $\Sigma_{3}^{0}$ predicate, a contradiction of its $\Pi_{3}^{0}$-completeness. Therefore $\mathcal{X} \notin \Sigma_{3}^{0}$ and we have established $\Sigma_{n}^{0} \subsetneq \Sigma_{n}^{0}\left[\Pi_{1}^{0}\right]$.

The inclusion $\Sigma_{n}^{0}\left[\Pi_{1}^{0}\right] \subseteq \Sigma_{n+1}^{0}$ is immediate from the definitions using $\Sigma_{n+1}^{0}=\Sigma_{n+1}^{0}\left[\Delta_{1}^{0}\right]$. That it is proper follows from the facts $\Sigma_{n+1}^{0}-\boldsymbol{\Sigma}_{\mathbf{n}}^{\mathbf{0}} \neq \emptyset$ and $\Sigma_{n}^{0}\left[\Pi_{1}^{0}\right] \subseteq \boldsymbol{\Sigma}_{\mathbf{n}}^{\mathbf{0}}$.

Intuitively, the classes $\Sigma_{1}^{0}\left[\Pi_{1}^{0}\right], \Pi_{1}^{0}\left[\Sigma_{1}^{0}\right], \Sigma_{2}^{0}\left[\Sigma_{1}^{0}\right], \Pi_{2}^{0}\left[\Pi_{1}^{0}\right], \ldots$ are slightly more powerful than their respective counterparts in the arithmetical hierarchy because they use one additional quantifier that is limited to the predicate.

\subsection{Correspondence Principles}

In this section we will prove that $\operatorname{cdim}(X)=\operatorname{dim}_{H}(X)$ for any $X$ that is an arbitrary union of $\Pi_{1}^{0}$-definable sets. We will also show that $\operatorname{dim}_{\text {comp }}(X)=\operatorname{dim}_{H}(X)$ if $X$ is $\Sigma_{2}^{0}$-definable.

Lemma 4.2. If $X \in \Pi_{1}^{0}$, then $\operatorname{dim}_{\mathrm{H}}(X)=\operatorname{dim}_{\text {comp }}(X)$.

Proof. Let $X \in \Pi_{1}^{0}$. Since $\operatorname{dim}_{\text {comp }}(X) \geq \operatorname{dim}_{\mathrm{H}}(X)$, it is enough to prove that $\operatorname{dim}_{\text {comp }}(X) \leq$ $\operatorname{dim}_{H}(X)$. For this, let $s>\operatorname{dim}_{\mathrm{H}}(X)$ be such that $2^{s}$ is rational. 
Since $s>\operatorname{dim}_{H}(X)$, for each $r \in \mathbb{N}$, there is a prefix set $A_{r} \subseteq\{0,1\}^{*}$ such that

$$
\sum_{w \in A_{r}} 2^{-s|w|} \leq 2^{-r} \text { and } X \subseteq \bigcup_{w \in A_{r}} \mathbf{C}_{w}
$$

Because $\mathbf{C}$ is compact and $X$ is closed, $X$ is compact. Thus each $A_{r}$ may be taken finite.

Because $X \in \Pi_{1}^{0}$, there is a computable function $h: \mathbb{N} \rightarrow\{0,1\}^{*} \cup\{\top\}$ such that

$$
X=\bigcap_{i=0}^{\infty} \mathbf{C}_{h(i)}^{c}
$$

For each $k \in \mathbb{N}$, let

$$
X_{k}=\bigcap_{i=0}^{k} \mathbf{C}_{h(i)}^{c} .
$$

Then for each $k \in \mathbb{N}$, it is easy to compute a finite prefix set $B_{k}$ such that

$$
\sum_{w \in B_{k}} 2^{-s|w|} \text { is minimal and } X_{k} \subseteq \bigcup_{w \in B_{k}} \mathbf{C}_{w}
$$

For each $r \in \mathbb{N}$, let

$$
k_{r}=\min \left\{k \mid \sum_{w \in B_{k}} 2^{-s|w|} \leq 2^{-r}\right\} .
$$

We know that each $k_{r}$ exists because of the existence of the finite prefix sets $A_{r}$ that satisfy the condition. Also, each $k_{r}$ can be computed by computing the finite sets $B_{k}$ until the condition is satisfied.

The rest of the proof is based on a construction that was used in [30] to prove Theorem 3.5, the gale characterization of Hausdorff dimension. There the prefix sets $A_{r}$ mentioned above are used to give an s-gale that succeeds on $X$. Here we use the finite, computable prefix sets $B_{k_{r}}$ in the same manner to give a computable s-gale that succeeds on $X$. 
Define for each $r \in \mathbb{N}$ a function $d_{r}:\{0,1\}^{*} \rightarrow[0, \infty)$ by

$$
d_{r}(w)= \begin{cases}2^{(s-1)(|w|-|v|)} & \text { if }(\exists v \sqsubseteq w) v \in B_{k_{r}} \\ \sum_{\substack{u \in\{0,1\}^{*} \\ w u \in B_{k_{r}}}} 2^{-s|u|} & \text { otherwise. }\end{cases}
$$

Then each $d_{r}$ is an s-gale. Also, $d_{r}(\lambda) \leq 2^{-r}$ and $d_{r}(w)=1$ for all $w \in B_{k_{r}}$. Next define a function $d$ on $\{0,1\}^{*}$ by $d=\sum_{r=0}^{\infty} 2^{r} d_{2 r}$. Then

$$
d(\lambda)=\sum_{r=0}^{\infty} 2^{r} d_{2 r}(\lambda) \leq \sum_{r=0}^{\infty} 2^{r} 2^{-2 r}=2
$$

so by induction it follows that $d(w)<\infty$ for all strings $w$. Therefore, by linearity, $d$ is an s-gale.

Let $S \in X$. Then $S \in X_{k_{2 r}}$ for all $r \in \mathbb{N}$. This means that $S$ has some prefix $S\left\lceil n \in B_{k_{2 r}}\right.$, and then

$$
d(S \uparrow n) \geq 2^{r} d_{2 r}(S \uparrow n)=2^{r} .
$$

Therefore $d$ succeeds on $S$, so $X \subseteq S^{\infty}[d]$.

To see that $d$ is computable, define $\hat{d}: \mathbb{N} \times\{0,1\}^{*} \rightarrow[0, \infty)$ by

$$
\hat{d}(i, w)=\sum_{r=0}^{\lceil s|w|\rceil+i} 2^{r} d_{2 r}(w) .
$$


We can exactly compute $\hat{d}$ by using the function $h$ to uniformly compute the sets $B_{k_{r}}$. Then

$$
\begin{aligned}
|d(w)-\hat{d}(i, w)| & =\sum_{r=\lceil s|w|\rceil+i+1}^{\infty} 2^{r} d_{2 r}(w) \\
& \leq \sum_{r=\lceil s|w|\rceil+i+1}^{\infty} 2^{r} 2^{s|w|} d_{2 r}(\lambda) \\
& \leq \sum_{r=\lceil s|w|\rceil+i+1}^{\infty} 2^{r+s|w|} 2^{-2 r} \\
& =2^{s|w|} \sum_{r=\lceil s|w|\rceil+i+1}^{\infty} 2^{-r} \\
& =2^{s|w|} 2^{-\lceil s|w|\rceil-i} \\
& \leq 2^{-i}
\end{aligned}
$$

so $\hat{d}$ is a computable approximation of $d$. Therefore $d$ is computable, so it witnesses that $\operatorname{dim}_{\text {comp }}(X) \leq s$. Since $s>\operatorname{dim}_{\mathrm{H}}(X)$ is arbitrary with $2^{s}$ rational and the rationals are dense, it follows that $\operatorname{dim}_{\text {comp }}(X) \leq \operatorname{dim}_{H}(X)$.

We now use the preceding lemma to give our correspondence principle for constructive dimension.

Theorem 4.3. If $X \subseteq \mathbf{C}$ is a union of $\Pi_{1}^{0}$ sets, then $\operatorname{dim}_{\mathrm{H}}(X)=\operatorname{cdim}(X)$.

Proof. Let $\mathcal{I}$ be an arbitrary index set, $X_{\alpha} \in \Pi_{1}^{0}$ for each $\alpha \in \mathcal{I}$, and $X=\bigcup_{\alpha \in \mathcal{I}} X_{\alpha}$. By definition, $\operatorname{dim}_{\mathrm{H}}(X) \leq \operatorname{cdim}(X)$. Using Theorem 3.13 (the pointwise stability of constructive dimension), Lemma 4.2, and the monotonicity of Hausdorff dimension, we have

$$
\begin{aligned}
\operatorname{cdim}(X) & =\sup _{\alpha \in \mathcal{I}} \operatorname{cdim}\left(X_{\alpha}\right) \\
& =\sup _{\alpha \in \mathcal{I}} \operatorname{dim}_{\mathrm{H}}\left(X_{\alpha}\right) \\
& \leq \operatorname{dim}_{\mathrm{H}}(X) .
\end{aligned}
$$


Theorem 4.3 yields a pointwise characterization of the classical Hausdorff dimension of unions of $\Pi_{1}^{0}$ sets.

Corollary 4.4. If $X \subseteq \mathbf{C}$ is a union of $\Pi_{1}^{0}$ sets, then

$$
\operatorname{dim}_{\mathrm{H}}(X)=\sup _{S \in X} \operatorname{dim}(S)
$$

Proof. This follows immediately from Theorems 4.3 and 3.13 .

If we require that the union in Theorem 4.3 be effective, we arrive at the following correspondence principle for computable dimension. This result also follows implicitly from Staiger's work on martingale exponents of increase [57].

Theorem 4.5. If $X \in \Sigma_{2}^{0}$, then $\operatorname{dim}_{\mathrm{H}}(X)=\operatorname{dim}_{\text {comp }}(X)$.

Proof. Let $X \in \Sigma_{2}^{0}$. Since $\operatorname{dim}_{\text {comp }}(X) \geq \operatorname{dim}_{\mathrm{H}}(X)$, it is enough to prove that $\operatorname{dim}_{\text {comp }}(X) \leq$ $\operatorname{dim}_{H}(X)$. For this, let $s>\operatorname{dim}_{H}(X)$ be such that $2^{s}$ is rational. As in the proof of the Lemma 4.2 , it suffices to give a computable $s$-gale $d$ that succeeds on $X$.

Since $X \in \Sigma_{2}^{0}$, there is a computable function $h: \mathbb{N} \times \mathbb{N} \rightarrow\{0,1\}^{*} \cup\{\top\}$ such that

$$
X=\bigcup_{j=0}^{\infty} \bigcap_{i=0}^{\infty} \mathbf{C}_{h(i, j)}^{c}
$$

For each $j \in \mathbb{N}$, let

$$
X_{j}=\bigcap_{i=0}^{\infty} \mathbf{C}_{h(i, j)}^{c} .
$$

Since each $X_{j} \subseteq X, \operatorname{dim}_{\mathrm{H}}\left(X_{j}\right) \leq \operatorname{dim}_{\mathrm{H}}(X)<s$. Each $X_{j} \in \Pi_{1}^{0}$, so by Lemma 4.2 , for each $j \in \mathbb{N}$, there is a computable s-gale $d_{j}$ with $d_{j}(\lambda) \leq 1$ that succeeds on $X_{j}$. Let $d=\sum_{j=0}^{\infty} 2^{-j} d_{j}$. Then $d$ is an $s$-gale, $d$ is computable by using $h$ to uniformly compute the $d_{j}$, and $X \subseteq S^{\infty}[d]$.

We note that Theorems 4.3 and 4.5 cannot be extended to higher levels of the arithmetical hierarchy. 
Observation 4.6. There is a set $X \in \Pi_{2}^{0}$ such that $\operatorname{dim}_{\mathrm{H}}(X) \neq \operatorname{cdim}(X)$.

Proof. It is well known that there exists a sequence $S \in \operatorname{RAND} \cap \Delta_{2}^{0}$. (A sequence $S$ is in $\Delta_{2}^{0}$ if $S$ is decidable relative to an oracle for the halting problem.) Let $X=\{S\}$. Since $S \in \Delta_{2}^{0}$, we have $X \in \Pi_{2}^{0}$. By Proposition 3.10, $\operatorname{cdim}(X)=\operatorname{dim}(S)=1$. But any singleton has Hausdorff dimension 0 , so $\operatorname{dim}_{\mathrm{H}}(X)=0$.

\subsection{Classification of Dimension Classes}

In this section we investigate the arithmetical complexity of the following dimension and strong dimension classes.

$$
\begin{aligned}
\operatorname{DIM}^{\alpha} & =\{A \in \mathbf{C} \mid \operatorname{dim}(A)=\alpha\} \\
\mathrm{DIM}^{\leq \alpha} & =\{A \in \mathbf{C} \mid \operatorname{dim}(A) \leq \alpha\} \\
\mathrm{DIM}^{\geq \alpha} & =\{A \in \mathbf{C} \mid \operatorname{dim}(A) \geq \alpha\} \\
\operatorname{DIM}_{\mathrm{str}}^{\alpha} & =\{A \in \mathbf{C} \mid \operatorname{Dim}(A)=\alpha\} \\
\mathrm{DIM}_{\mathrm{str}}^{\leq \alpha} & =\{A \in \mathbf{C} \mid \operatorname{Dim}(A) \leq \alpha\} \\
\mathrm{DIM}_{\text {str }}^{\geq \alpha} & =\{A \in \mathbf{C} \mid \operatorname{Dim}(A) \geq \alpha\}
\end{aligned}
$$

Let $\alpha \in[0,1]$ be $\Delta_{2}^{0}$-computable. For any such $\alpha$, it is well known that there is a computable function $\hat{\alpha}: \mathbb{N} \rightarrow \mathbb{Q}$ such that $\lim _{n \rightarrow \infty} \hat{\alpha}(n)=\alpha$. Using Theorem 3.14, we have

$$
\begin{aligned}
\operatorname{dim}(X) \leq \alpha & \Longleftrightarrow \liminf _{n \rightarrow \infty} \frac{K(X\lceil n)}{n} \leq \alpha \\
& \Longleftrightarrow(\forall k)(\forall N)(\exists n \geq N) K(X\lceil n)<(\hat{\alpha}(n)+1 / k) n,
\end{aligned}
$$

so $\mathrm{DIM}^{\leq \alpha}$ is a $\Pi_{2}^{0}$-class. Also,

$$
\begin{aligned}
\operatorname{dim}(X) \geq \alpha & \Longleftrightarrow \liminf _{n \rightarrow \infty} \frac{K(X\lceil n)}{n} \geq \alpha \\
& \Longleftrightarrow(\forall k)(\exists N)(\forall n \geq N) K(X\lceil n)>(\hat{\alpha}(N)-1 / k) n
\end{aligned}
$$


so $\mathrm{DIM}^{\geq \alpha}$ is a $\Pi_{3}^{0}$-class. Therefore we have the following.

Proposition 4.7. 1. The class $\mathrm{DIM}^{0}$ is $\Pi_{2}^{0}$.

2. For all $\Delta_{2}^{0}$-computable $\alpha \in(0,1], \mathrm{DIM}^{\alpha}$ is a $\Pi_{3}^{0}$-class.

3. For arbitrary $\alpha \in(0,1], \mathrm{DIM}^{\alpha}$ is a $\mathbf{\Pi}_{3}^{\mathbf{0}}$-class.

The situation is slightly more complicated for strong dimension. By Theorem 3.14, we have

$$
\begin{aligned}
\operatorname{Dim}(X) \leq \alpha & \Longleftrightarrow \quad \limsup _{n \rightarrow \infty} \frac{K(X \uparrow n)}{n} \leq \alpha \\
& \Longleftrightarrow \quad(\forall k)(\exists N)(\forall n \geq N) K(X\lceil n)<(\hat{\alpha}(N)+1 / k) n \\
& \Longleftrightarrow \quad(\forall k)(\exists N)(\forall n \geq N)(\exists\langle\pi, t\rangle)|\pi|<(\hat{\alpha}(N)+1 / k) n \\
& \quad \text { and } U(\pi)=X\lceil n \text { in } \leq t \text { computation steps }
\end{aligned}
$$

where $U$ is the fixed universal self-delimiting Turing machine used to define $K$. From this it is clear that $\operatorname{DIM}_{\mathrm{str}}^{\leq \alpha} \in \Pi_{4}^{0}$. However, the " $(\exists\langle\pi, t\rangle)$ " quantifier is local to the defining predicate, so we have $\operatorname{DIM}_{\text {str }}^{\leq \alpha} \in \mathbf{\Pi}_{\mathbf{3}}^{\mathbf{0}}$, and in fact, it is a $\Pi_{3}^{0}\left[\Sigma_{1}^{0}\right]$-class. Also,

$$
\begin{aligned}
\operatorname{Dim}(X) \geq \alpha & \Longleftrightarrow \quad \limsup _{n \rightarrow \infty} \frac{K(X\lceil n)}{n} \geq \alpha \\
& \Longleftrightarrow \quad(\forall k)(\forall N)(\exists n \geq N) K(X\lceil n)>(\hat{\alpha}(n)-1 / k) n,
\end{aligned}
$$

so $\operatorname{DIM}_{\text {str }}^{\geq \alpha}$ is a $\Pi_{2}^{0}\left[\Pi_{1}^{0}\right]$-class. This establishes the following analogue of Proposition 4.7.

Proposition 4.8. 1. The class $\operatorname{DIM}_{\mathrm{str}}^{1}$ is $\Pi_{2}^{0}\left[\Pi_{1}^{0}\right]$.

2. For all $\Delta_{2}^{0}$-computable $\alpha \in[0,1), \mathrm{DIM}_{\mathrm{str}}^{\alpha}$ is a $\Pi_{3}^{0}\left[\Sigma_{1}^{0}\right]$-class.

3. For arbitrary $\alpha \in[0,1), \mathrm{DIM}_{\mathrm{str}}^{\alpha}$ is a $\mathbf{\Pi}_{\mathbf{3}}^{\mathbf{0}}$-class.

In the remainder of this section we prove that the classifications in Propositions 4.7 and 4.8 cannot be improved in their respective hierarchies. 


\subsubsection{Category Methods}

Recall that a class $\mathcal{X}$ is meager if it is included in a countable union of nowhere dense subsets of $\mathbf{C}$, and comeager if its complement $\overline{\mathcal{X}}$ is meager. The following lemma (implicit in Rogers [48, p341]) will be useful.

Lemma 4.9. If $\mathcal{X} \in \mathbf{\Sigma}_{\mathbf{2}}^{0}$ and $\overline{\mathcal{X}}$ is dense then $\mathcal{X}$ is meager.

Proof. Suppose that $\mathcal{X}=\bigcup_{n} \mathcal{X}_{n}, \mathcal{X}_{n}$ closed. Since $\overline{\mathcal{X}}$ is dense, $\mathcal{X}_{n}$ contains no basic open set, hence $\mathcal{X}_{n}$ is nondense (i.e. its closure contains no basic open set), and $\mathcal{X}$ is a countable union of nondense sets.

The class RAND of Martin-Löf random sets can easily be classified with category methods.

Theorem 4.10. RAND is a $\Sigma_{2}^{0}$-class, but not a $\mathbf{\Pi}_{\mathbf{2}}^{0}$-class.

Proof. This is a well known result. The proof is analogous to the one in Rogers [48, p 341] that $\{X \mid X$ finite $\}$ is a $\Sigma_{2}^{0}$-class but not a $\Pi_{2}^{0}$-class. Both RAND and its complement are dense, so by Lemma 4.9, RAND is meager. If RAND were a $\mathbf{\Pi}_{\mathbf{2}}^{\mathbf{0}}$-class, then again using Lemma 4.9, its complement would also be meager. This contradicts the fact that $\mathbf{C}$ is not meager.

As $\mathrm{DIM}^{0}$ and $\mathrm{DIM}_{\text {str }}^{1}$ are dense $\boldsymbol{\Pi}_{\mathbf{2}}^{\mathbf{0}}$-classes that have dense complements, an argument similar to the one used for Theorem 4.10 shows that they are not $\boldsymbol{\Sigma}_{\mathbf{2}}^{\mathbf{0}}$-classes.

Theorem 4.11. The classes $\mathrm{DIM}^{0}$ and $\mathrm{DIM}_{\mathrm{str}}^{1}$ are not $\boldsymbol{\Sigma}_{\mathbf{2}}^{\mathbf{0}}$-classes.

We now develop category methods for the other $\mathrm{DIM}^{\alpha}$ classes. Following Schnorr [53], we call an unbounded nondecreasing function $h:\{0,1\}^{*} \rightarrow\{0,1\}^{*}$ an order. For any supermartingale $d$, the order $h$ success set of $d$ is

$$
S^{h}[d]=\left\{A \in \mathbf{C} \mid \limsup _{n \rightarrow \infty} \frac{d(A \uparrow n)}{h(n)} \geq 1\right\}
$$

Let $\mathbf{d}$ be a multiplicatively optimal constructive supermartingale (for example, $\mathbf{d}^{(1)}$ from Theorem 3.11). For every rational $s$, define the computable order $h_{s}(n)=2^{(1-s) n}$. From 
Theorem 3.15 it follows that for any $\mathcal{A} \subseteq \mathbf{C}$,

$$
\left.\operatorname{cdim}(\mathcal{A})=\inf \left\{s \in \mathbb{Q}: \mathcal{A} \subseteq S^{h_{s}}[\mathbf{d}]\right)\right\}
$$

Lemma 4.12. For every rational $s \in(0,1), S^{h_{s}}[\mathbf{d}]$ is a comeager $\Pi_{2}^{0}$-class.

Proof. Notice that $\overline{S^{h_{s}}[\mathbf{d}]} \in \Sigma_{2}^{0}$ and $S^{h_{s}}[\mathbf{d}]$ is dense. Now apply Lemma 4.9.

Lemma 4.13. For all $\alpha \in(0,1], \mathrm{DIM}^{\alpha}$ is meager.

Proof. Let $s<\alpha$ be rational. Lutz [31] showed that $\mathbf{d}^{(s)}(w)=2^{(s-1)|w|} \mathbf{d}(w)$ is an optimal constructive $s$-supergale. It follows that for any $A \in \mathbf{C}, A \in S^{h_{s}}[\mathbf{d}] \Rightarrow \operatorname{dim}(S)<\alpha$. Therefore $\mathrm{DIM}^{\alpha} \subseteq \overline{S^{h_{s}}}$, so $\mathrm{DIM}^{\alpha}$ is meager by Lemma 4.12 .

Proposition 4.14. For all $\alpha \in(0,1], \mathrm{DIM}^{\alpha}$ is not a $\Pi_{2}^{0}$-class.

Proof. If $\mathrm{DIM}^{\alpha} \in \Pi_{2}^{0}$, then Lemma 4.9 implies that $\mathrm{DIM}^{\alpha}$ is comeager, contradicting Lemma 4.13 .

To strengthen Proposition 4.14 to show that $\mathrm{DIM}^{\alpha}$ is not $\Sigma_{3}^{0}$, we now turn to Wadge reductions.

\subsubsection{Wadge Reductions}

Let $\mathcal{A}, \mathcal{B} \subseteq \mathbf{C}$. A Wadge reduction of $\mathcal{A}$ to $\mathcal{B}$ is a function $f: \mathbf{C} \rightarrow \mathbf{C}$ that is continuous and satisfies $\mathcal{A}=f^{-1}(\mathcal{B})$, i.e., $X \in \mathcal{A} \Longleftrightarrow f(X) \in \mathcal{B}$. We say that $\mathcal{B}$ is Wadge complete for a class $\boldsymbol{\Gamma}$ of subsets of $\mathbf{C}$ if $\mathcal{B} \in \boldsymbol{\Gamma}$ and every $\mathcal{A} \in \boldsymbol{\Gamma}$ Wadge reduces to $\mathcal{B}$. As the classes of the Borel hierarchy are closed under Wadge reductions, Wadge completeness can be used to properly identify the location of a subset of $\mathbf{C}$ in the hierarchy.

We now prove that $\mathrm{DIM}^{1}$ is Wadge complete for $\boldsymbol{\Pi}_{\mathbf{3}}^{\mathbf{0}}$. We will then give Wadge reductions from it to $\mathrm{DIM}^{\alpha}$ for the other values of $\alpha$.

Theorem 4.15. $\mathrm{DIM}^{1}$ is Wadge complete for $\boldsymbol{\Pi}_{\mathbf{3}}^{\mathbf{0}}$. Therefore $\mathrm{DIM}^{1}$ is not a $\boldsymbol{\Sigma}_{\mathbf{3}}^{\mathbf{0}}$-class, and in particular is not a $\Sigma_{3}^{0}$-class. 
Proof. One could prove this by reducing a known $\boldsymbol{\Pi}_{\mathbf{3}}^{\mathbf{0}}$-complete class to DIM ${ }^{1}$, e.g. the class of sets that have a limiting frequency of 1 's that is 0 (this class was proved to be $\boldsymbol{\Pi}_{\mathbf{3}}^{\mathbf{0}}$-complete by $\mathrm{Ki}$ and Linton [26]), but it is just as easy to build a direct reduction from an arbitrary $\Pi_{3}^{0}$-class.

Let $\mathbf{d}$ be a multiplicatively optimal constructive supermartingale. Note that we have

$$
S^{2^{n}}[\mathbf{d}] \subsetneq \ldots \subsetneq S^{2^{\frac{1}{k} n}}[\mathbf{d}] \subsetneq S^{2^{\frac{1}{k+1} n}}[\mathbf{d}] \subsetneq \ldots \subsetneq \mathrm{DIM}^{1}
$$

Let $\bigcup_{k} \bigcap_{s} \mathcal{O}_{k, s}$ be a $\boldsymbol{\Sigma}_{\mathbf{3}}^{0}$-class. Without loss of generality $\mathcal{O}_{k, s} \supseteq \mathcal{O}_{k, s+1}$ for all $k, s$. We define a continuous function $f: \mathbf{C} \rightarrow \mathbf{C}$ such that

$$
\forall k\left(X \in \bigcap_{s} \mathcal{O}_{k, s} \Longleftrightarrow f(X) \in S^{2^{\frac{1}{k} n}}[\mathbf{d}]\right)
$$

so that we have

$$
\begin{aligned}
X \notin \bigcup_{k} \bigcap_{s} \mathcal{O}_{k, s} & \Longleftrightarrow \forall k\left(f(X) \notin S^{2^{\frac{1}{k} n}}[\mathbf{d}]\right) \\
& \Longleftrightarrow f(X) \in \mathrm{DIM}^{1} .
\end{aligned}
$$

The image $Y=f(X)$ is defined in stages, $Y=\bigcup_{s} Y_{s}$, such that every initial segment of $X$ defines an initial segment of $Y$.

At stage 0 we define $Y_{0}$ to be the empty sequence.

At stage $s>0$ we consider $X\left\lceil s\right.$, and for each $k$ we define $t_{k, s}$ to be the largest stage $t \leq s$ such that $X\left\lceil s \in \mathcal{O}_{k, t}\right.$. (Let $t_{k, s}=0$ if such a $t$ does not exist.) Define $k$ to be expansionary at stage $s$ if $t_{k, s-1}<t_{k, s}$. Now we let $k(s)=\min \{k: k$ is expansionary at $s\}$. There are two substages.

Substage (a). First consider all strings $\sigma$ extending $Y_{s-1}$ of minimal length with $\mathbf{d}(\sigma) \geq$ $2^{\frac{1}{k(s)}|\sigma|}$, and take the leftmost one of these $\sigma$ 's. Such $\sigma$ 's exist because $S^{2^{\frac{1}{k(s)} n}}[\mathbf{d}]$ is dense. If $k(s)$ does not exist, let $\sigma=Y_{s-1}$.

Substage (b). Next consider all extensions $\tau \sqsupseteq \sigma$ of minimal length such that $\mathbf{d}(\tau$ † 
$i) \leq \mathbf{d}(\tau \uparrow(i-1))$ for every $|\sigma|<i<|\tau|$, and $\mathbf{d}(\tau) \leq|\tau|$. Clearly such $\tau$ exist, by direct diagonalization against $\mathbf{d}$. Define $Y_{s}$ to be the leftmost of these $\tau$. This concludes the construction.

So $Y_{s}$ is defined by first building a piece of evidence $\sigma$ that $\mathbf{d}$ achieves growth rate $2^{\frac{1}{k(s)} n}$ on $Y$ and then slowing down the growth rate of $\mathbf{d}$ to the order $n$. Note that $f$ is continuous. If $X \in \bigcup_{k} \bigcap_{s} \mathcal{O}_{k, s}$, then for the minimal $k$ such that $X \in \bigcap_{s} \mathcal{O}_{k, s}$, infinitely many pieces of evidence $\sigma$ witness that $\mathbf{d}$ achieves growth rate $2^{\frac{1}{k} n}$ on $Y$, so $Y \notin \mathrm{DIM}^{1}$. On the other hand, if $X \notin \bigcup_{k} \bigcap_{s} \mathcal{O}_{k, s}$ then for every $k$ only finitely often $\mathbf{d}\left(Y_{s}\right) \geq 2^{\frac{1}{k}\left|Y_{s}\right|}$ because in substage (a) the extension $\sigma$ is chosen to be of minimal length, so $Y \notin S_{h_{k}}[\mathbf{d}]$. Hence $Y \in \mathrm{DIM}^{1}$.

As RAND is a $\Sigma_{2}^{0}$-class, we have the following corollary (which can also be proved by a direct construction).

Corollary 4.16. (Lutz [31]) RAND is a proper subset of $\mathrm{DIM}^{1}$.

In order to establish the existence of $\Delta_{2}^{0}$-computable sequences of any $\Delta_{2}^{0}$-computable dimension $\alpha \in[0,1)$, Lutz [31] defined a dilution function $g_{\alpha}: \mathbf{C} \rightarrow \mathbf{C}$ that is computable and satisfies $\operatorname{dim}\left(g_{\alpha}(X)\right)=\alpha \cdot \operatorname{dim}(X)$ for all $X \in \mathbf{C}$. Applying this to any $\Delta_{2}^{0}$-computable Martin-Löf random sequence (which must have dimension 1) establishes the existence theorem. (We note that $g_{\alpha}(X)$ has the same Turing degree as $X$. Since by the Low Basis Theorem of Jockusch and Soare [46, Theorem V.5.32] there are Martin-Löf random sets of low degree, we immediately obtain that there are low sets of any $\Delta_{2}^{0}$-computable dimension $\alpha$.) As $g_{\alpha}$ is continuous, it is a Wadge reduction from $\mathrm{DIM}^{1}$ to $\mathrm{DIM}^{\alpha}$ if $\alpha>0$. Combining this with the previous theorem, we have that $\mathrm{DIM}^{\alpha}$ is Wadge complete for $\mathbf{\Pi}_{\mathbf{3}}^{\mathbf{0}}$ for all $\Delta_{2}^{0}$-computable $\alpha \in(0,1)$. We now give a similar dilution construction that will allow us to prove this for arbitrary $\alpha \in(0,1)$.

Let $X \in \mathbf{C}$ and let $\alpha \in(0,1)$. Write $X=x_{1} x_{2} x_{3} \ldots$ where $\left|x_{n}\right|=2 n-1$ for all $n$, noting that $\left|x_{1} \cdots x_{n}\right|=n^{2}$. For each $n$, let

$$
k_{n}=\left\lceil n \frac{1-\alpha}{\alpha}\right\rceil
$$


and $y_{n}=0^{k_{n}}$. We then define

$$
f_{\alpha}(X)=x_{1} y_{1} x_{2} y_{2} \cdots x_{n} y_{n} \cdots .
$$

Observe that $f_{\alpha}$ is a continuous function mapping $\mathbf{C}$ to $\mathbf{C}$. We now show that it modifies the dimension of $X$ in a controlled manner.

Lemma 4.17. For any $X \in \mathbf{C}$ and $\alpha \in(0,1)$,

$$
\operatorname{dim}\left(f_{\alpha}(X)\right)=\alpha \cdot \operatorname{dim}(X)
$$

and

$$
\operatorname{Dim}\left(f_{\alpha}(X)\right)=\alpha \cdot \operatorname{Dim}(X) .
$$

Proof. The proof uses Theorem 3.14, the Kolmogorov complexity characterizations of dimension and strong dimension.

Let $w \sqsubseteq f_{\alpha}(X)$. For some $n$,

$$
w=x_{1} y_{1} \cdots x_{n-1} y_{n-1} v
$$

where $v \sqsubseteq x_{n} y_{n}$. Then

$$
\begin{aligned}
K(w) \leq & K\left(x_{1} \cdots x_{n-1}\right)+K(v) \\
& +K\left(k_{1}\right)+\cdots+K\left(k_{n-1}\right)+O(1) \\
\leq & K\left(x_{1} \cdots x_{n-1}\right)+O(n \log n) .
\end{aligned}
$$

Because

$$
|w| \geq\left|x_{1} y_{1} \cdots x_{n-1} y_{n-1}\right| \geq \frac{(n-1)^{2}}{\alpha},
$$

we have

$$
\frac{K(w)}{|w|} \leq \frac{\alpha \cdot K\left(x_{1} \cdots x_{n-1}\right)}{\left|x_{1} \cdots x_{n-1}\right|}+\frac{O(n \log n)}{(n-1)^{2}}
$$


It follows that

$$
\begin{aligned}
\operatorname{dim}\left(f_{\alpha}(X)\right) & \leq \alpha \liminf _{n \rightarrow \infty} \frac{K\left(x_{1} \cdots x_{n-1}\right)}{\left|x_{1} \cdots x_{n-1}\right|} \\
& =\alpha \liminf _{n \rightarrow \infty} \frac{K(x\lceil n)}{n} \\
& =\alpha \cdot \operatorname{dim}(X)
\end{aligned}
$$

where the first equality holds because the blocks $x_{n}$ are short relative to $x_{1} \cdots x_{n-1}$. Similarly, $\operatorname{Dim}\left(f_{\alpha}(X)\right) \leq \alpha \cdot \operatorname{Dim}(X)$.

For the other inequality, we have

$$
\begin{aligned}
K\left(x_{1} \cdots x_{n-1}\right) \leq & K(w)+K\left(k_{1}\right)+\cdots+K\left(k_{n-1}\right) \\
& +O(1) \\
\leq & K(w)+O(n \log n)
\end{aligned}
$$

and

$$
|w| \leq\left|x_{1} y_{1} \cdots x_{n} y_{n}\right| \leq \frac{n^{2}}{\alpha}+n \leq \frac{(n+1)^{2}}{\alpha}
$$

so

$$
\begin{aligned}
\frac{K(w)}{|w|} & \geq \alpha \frac{K\left(x_{1} \cdots x_{n-1}\right)-O(n \log n)}{(n+1)^{2}} \\
& =\alpha \frac{K\left(x_{1} \cdots x_{n-1}\right)}{\left|x_{1} \cdots x_{n-1}\right|} \frac{(n-1)^{2}}{(n+1)^{2}}-\frac{O(n \log n)}{(n+1)^{2}}
\end{aligned}
$$

Therefore

$$
\begin{aligned}
\operatorname{dim}\left(f_{\alpha}(X)\right) & \geq \alpha \liminf _{n \rightarrow \infty} \frac{K\left(x_{1} \cdots x_{n-1}\right)}{\left|x_{1} \cdots x_{n-1}\right|} \\
& =\alpha \liminf _{n \rightarrow \infty} \frac{K(x\lceil n)}{n} \\
& =\alpha \cdot \operatorname{dim}(X)
\end{aligned}
$$


and analogously, $\operatorname{Dim}\left(f_{\alpha}(X)\right) \geq \alpha \cdot \operatorname{Dim}(X)$.

The function $f_{\alpha}$ establishes the completeness of $\mathrm{DIM}^{\alpha}$.

Theorem 4.18. For all $\alpha \in(0,1), \mathrm{DIM}^{\alpha}$ is Wadge complete for $\mathbf{\Pi}_{\mathbf{3}}^{\mathbf{0}}$. Therefore it is not a $\Sigma_{\mathbf{3}}^{\mathbf{0}}$-class, and in particular not a $\Sigma_{3}^{0}$-class.

Proof. By Lemma 4.17, $f_{\alpha}$ is a Wadge reduction from $\mathrm{DIM}^{1}$ to $\mathrm{DIM}^{\alpha}$. Therefore $\mathrm{DIM}^{\alpha}$ is Wadge complete for $\boldsymbol{\Pi}_{\mathbf{3}}^{\mathbf{0}}$ by composing $f_{\alpha}$ with the reduction from Theorem 4.15.

As $g_{\alpha}$ is also a Wadge reduction from $\mathrm{DIM}_{\text {str }}^{1}$ to $\mathrm{DIM}_{\mathrm{str}}^{\alpha}$, we have from Theorem 4.11 that $\mathrm{DIM}_{\text {str }}^{\alpha}$ is not a $\boldsymbol{\Sigma}_{\mathbf{2}}^{\mathbf{0}}$-class for all $\alpha \in(0,1)$. We now prove that $\operatorname{DIM}_{\text {str }}^{\alpha}$ is not even $\boldsymbol{\Sigma}_{\mathbf{3}}^{\mathbf{0}}$ for all $\alpha \in[0,1)$.

Theorem 4.19. For all $\alpha \in[0,1), \mathrm{DIM}_{\mathrm{str}}^{\alpha}$ is Wadge complete for $\mathbf{\Pi}_{\mathbf{3}}^{0}$. Therefore $\mathrm{DIM}_{\mathrm{str}}^{\alpha}$ is not a $\boldsymbol{\Sigma}_{\mathbf{3}}^{\mathbf{0}}$-class, and in particular is not a $\Sigma_{3}^{0}\left[\Pi_{1}^{0}\right]$-class.

Proof. The proof is similar to that of Theorem 4.15, but uses the Kolmogorov complexity characterization of strong dimension (Theorem 3.14). Let $\mathcal{C}=\bigcup_{k} \bigcap_{s} \mathcal{O}_{k, s}$ be a $\boldsymbol{\Sigma}_{\mathbf{3}}^{\mathbf{0}}$-class and without loss of generality assume that $\mathcal{O}_{k, s} \supseteq \mathcal{O}_{k, s+1}$ for all $k, s$.

Let $\alpha \in(0,1)$. (We will discuss the simpler case $\alpha=0$ later.) We define a continuous function $f: \mathbf{C} \rightarrow \mathbf{C}$ in stages that will Wadge reduce $\mathcal{C}$ to $\overline{\operatorname{DIM}_{\text {str }}^{\alpha}}$. The image $Y=f(X)$ will be the unique sequence extending $Y_{s}$ for all $s$. At stage 0 we define $Y_{0}$ to be the empty sequence.

At stage $s>0$ we consider $X \uparrow s$, and define $k(s)$ as in the proof of Theorem 4.15. There are three substages.

Substage (a). First consider all strings $\rho$ extending $Y_{s-1}$ of minimal length with $K(\rho) \geq$ $\alpha|\rho|$, and take the leftmost one of these $\rho$ 's.

Substage (b). Next consider all strings $\sigma$ extending $\rho$ of minimal length with $K(\sigma) \geq$ $\left(\alpha+\frac{1}{k(s)}\right)|\sigma|$, and take the leftmost one of these $\sigma$ 's. If $k(s)$ does not exist, let $\sigma=\rho$.

Substage (c). Extend $\sigma$ with a block of 0's to obtain $Y_{s}=\sigma 0^{|\sigma|^{2}-|\sigma|}$. 
That is, to define $Y_{s}$, we first select $\rho$ to increase the Kolmogorov complexity rate to $\alpha$. This ensures that $Y$ will have strong dimension at least $\alpha$. We then construct a piece of evidence $\sigma$ that $Y$ has strong dimension at least $\alpha+\frac{1}{k(s)}$. We finish $Y_{s}$ with a long block of 0's to bring the Kolmogorov complexity down to a near-zero rate, so that the next stage will work properly.

If $X \in \mathcal{C}$, then for the minimal $k$ such that $X \in \bigcap_{s} \mathcal{O}_{k, s}$, infinitely many prefixes $\sigma \sqsubseteq Y$ satisfy $K(\sigma) \geq\left(\alpha+\frac{1}{k}\right)|\sigma|$. Therefore $\operatorname{Dim}(Y) \geq \alpha+\frac{1}{k}$, so $Y \notin \operatorname{DIM}_{\text {str }}^{\alpha}$.

Now let $X \notin \mathcal{C}$. Let $\alpha^{\prime}>\alpha$ be arbitrary, and choose $k$ so that $\frac{1}{k}<\alpha^{\prime}-\alpha$. Because $X \notin \mathcal{C}$, we have $k(s)>k$ for all sufficiently large $s$. Let $s_{0}$ be large enough to ensure $k(s)>s$ and $K\left(Y_{s-1}\right) \leq \sqrt{\left|Y_{s-1}\right|}+O(1)<\alpha\left|Y_{s-1}\right|$ hold for all $s \geq s_{0}$. Suppose that

$$
K(w) \geq \alpha^{\prime}|w|
$$

holds for some $w$ with $Y_{s-1} \sqsubseteq w \sqsubseteq Y_{s}$ for some stage $s \geq s_{0}$. We then have that $\rho$ is a proper extension of $Y_{s-1}$. By choice of $\rho$ and $\sigma$ and the fact that $\alpha^{\prime}>\alpha+\frac{1}{k}>\alpha+\frac{1}{k(s)}$, we must have $w=\rho$ or $\sigma \sqsubseteq w$. We analyze these two cases separately.

(i) $w=\rho$ : Let $\rho^{\prime}$ be the string obtained from $\rho$ by removing the last bit. Then $K(\rho) \leq$ $K\left(\rho^{\prime}\right)+O(1)$. By choice of $\rho$, we have $K\left(\rho^{\prime}\right)<\alpha\left|\rho^{\prime}\right|$. We also have $K(\rho) \geq\left(\alpha^{\prime}\right)|\rho|$ by (4.2). Putting these three statements together yields

$$
\alpha^{\prime}|\rho|<\alpha(|\rho|-1)+O(1)
$$

which is a contradiction if $|\rho|=|w|$ is sufficiently large.

(ii) $\sigma \sqsubseteq w$ : Obtain $\sigma^{\prime}$ from $\sigma$ by removing the last bit of $\sigma$. Then we have

$$
\begin{aligned}
K(w) & \leq K\left(\sigma^{\prime}\right)+K(|w|-|\sigma|)+O(1) \\
& \leq K\left(\sigma^{\prime}\right)+\log (|w|-|\sigma|)+O(1) \\
& \leq K\left(\sigma^{\prime}\right)+2 \log |\sigma|+O(1) .
\end{aligned}
$$


By choice of $\sigma, K\left(\sigma^{\prime}\right)<\left(\alpha+\frac{1}{k(s)}\right)\left|\sigma^{\prime}\right|$. These two facts together with (4.2) tell us that

$$
\alpha^{\prime}|w|<\left(\alpha+\frac{1}{k(s)}\right)(|\sigma|-1)+2 \log |\sigma|+O(1)
$$

which is a contradiction for large $|w|$ because $|w| \geq|\sigma|$ and $\alpha^{\prime}>\alpha+\frac{1}{k(s)}$.

Therefore, for all sufficiently long $w \sqsubseteq Y,(4.2)$ does not hold. It follows that $\operatorname{Dim}(Y) \leq \alpha$. On the other hand, there are infinitely many $\rho \sqsubseteq Y$ with $K(\rho) \geq \alpha|\rho|$, so $\operatorname{Dim}(Y) \geq \alpha$. Therefore $Y \in \mathrm{DIM}_{\mathrm{str}}^{\alpha}$.

This shows that $f$ is a Wadge reduction from $\mathcal{C}$ to $\overline{\operatorname{DIM}_{\text {str }}^{\alpha}}$. As $\mathcal{C}$ is an arbitrary $\boldsymbol{\Sigma}_{\mathbf{3}}^{0}$-class, this shows that $\mathrm{DIM}_{\mathrm{str}}^{\alpha}$ is Wadge complete for $\boldsymbol{\Pi}_{\mathbf{3}}^{\mathbf{0}}$.

The proof for the case $\alpha=0$ is similar, but simpler as substage (a) is omitted in the construction.

\subsubsection{Ad Hoc Methods}

When classifying classes in the arithmetical hierarchy of reals there are several methods one can use. As we have seen, category methods are sometimes useful up to the second level, Wadge reductions are useful if the classification in the effective (lightface) hierarchy coincides with that in the classical (boldface) hierarchy, and sometimes (as in Proposition 4.1 ) one just needs something else. In particular when the level of the class in the effective hierarchy is not the same as the level in the classical hierarchy one often needs to resort to ad hoc arguments. One might think that the notion of effective Wadge reduction, or recursive functional, would be the proper notion to use in classifying classes of reals in the effective hierarchy. However, this notion is rarely useful for the following reason. Let $\mathcal{X}$ be a class without computable elements, such as the class of Martin-Löf random sets or the class of 1-generic sets. Then $\mathcal{X}$ cannot be proven to be complete for any level of the effective hierarchy by a recursive Wadge reduction $f$. For if $X$ is recursive, then so is $f(X)$, so we can never have $X \in \mathbf{C} \Longleftrightarrow f(X) \in \mathcal{X}$. So we see that "easy" classes like $\mathbf{C}$ that contain recursive elements cannot be reduced in such a way to many "difficult" classes, which renders the notion rather useless. 
We have left open the question whether $\mathrm{DIM}_{\text {str }}^{1}$ is not in $\Pi_{2}^{0}$, and whether $\mathrm{DIM}_{\text {str }}^{\alpha}$ is not in $\Pi_{3}^{0}$ for any $\Delta_{2}^{0}$-computable $\alpha \in[0,1)$. We have no answer to the second question, but we provide an answer to the first in the next theorem. We make use of the following lemma:

Lemma 4.20. If $\mathcal{X} \in \Pi_{2}^{0}$ is dense then there is a computable $X \in \mathcal{X}$.

Proof. This is an easy finite extension argument. Suppose that $\mathcal{X}=\left\{X:(\forall m)(\exists k) R^{X}(m, k) \downarrow=\right.$ $1\} \in \Pi_{2}^{0}$ is dense. (Here $R$ is a computable predicate. Note that $R$ does not have to be defined with oracles $X$ that are not in $\mathcal{X}$.) Given any initial segment $\tau$ such that

$$
(\forall n<m)(\exists k) R^{\tau}(m, k) \downarrow=1,
$$

we show how to compute an extension $\sigma \sqsupset \tau$ such that

$$
(\exists k) R^{\sigma}(m, k) \downarrow=1 \text {. }
$$

Because $\mathcal{X}$ is dense, there are $X \sqsupset \tau$ and $k$ such that $R^{X}(m, k) \downarrow=1$. Let $u$ be the use of this computation, i.e. the part of the oracle $X$ used in it. Now define $\sigma=\max \{X\lceil u, \tau\}$. Then $\sigma \sqsupseteq \tau$ satisfies (4.3).

Now it is clear that for every $m$ we can compute appropriate extensions $\sigma_{m}$ such that $X=\bigcup_{m} \sigma_{m}$ is computable and $(\forall m)(\exists k) R^{\sigma_{m}}(m, k) \downarrow=1$, so that $X \in \mathcal{X}$.

Theorem 4.21. $\mathrm{DIM}_{\mathrm{str}}^{1}$ is not a $\Pi_{2}^{0}$-class. Hence it is properly $\Pi_{2}^{0}\left[\Pi_{1}^{0}\right]$.

Proof. Suppose that $\mathrm{DIM}_{\text {str }}^{1}$ is $\Pi_{2}^{0}$. Then, since clearly $\mathrm{DIM}_{\mathrm{str}}^{1}$ is dense, by Lemma 4.20 it contains a computable real, contradicting that every computable real has strong dimension 0 .

The results of this section are summarized in the following figure. 


\begin{tabular}{|c|c|c|}
\hline & $\mathrm{DIM}^{\alpha}$ & $\mathrm{DIM}_{\text {str }}^{\alpha}$ \\
\hline \hline$\alpha=0$ & $\Pi_{2}^{0}-\boldsymbol{\Sigma}_{\mathbf{2}}^{\mathbf{0}}$ & $\Pi_{3}^{0}\left[\Sigma_{1}^{0}\right]-\boldsymbol{\Sigma}_{\mathbf{3}}^{\mathbf{0}}$ \\
\hline$\alpha \in(0,1) \cap \Delta_{2}^{0}$ & $\Pi_{3}^{0}-\boldsymbol{\Sigma}_{\mathbf{3}}^{\mathbf{0}}$ & $\Pi_{3}^{0}\left[\Sigma_{1}^{0}\right]-\boldsymbol{\Sigma}_{\mathbf{3}}^{\mathbf{0}}$ \\
\hline$\alpha=1$ & $\Pi_{3}^{0}-\boldsymbol{\Sigma}_{\mathbf{3}}^{\mathbf{0}}$ & $\Pi_{2}^{0}\left[\Pi_{1}^{0}\right]-\left(\boldsymbol{\Sigma}_{\mathbf{2}}^{\mathbf{0}} \cup \Pi_{2}^{0}\right)$ \\
\hline arbitrary $\alpha \in(0,1)$ & $\mathbf{\Pi}_{\mathbf{3}}^{\mathbf{0}}-\boldsymbol{\Sigma}_{\mathbf{3}}^{\mathbf{0}}$ & $\boldsymbol{\Pi}_{\mathbf{3}}^{\mathbf{0}}-\boldsymbol{\Sigma}_{\mathbf{3}}^{\mathbf{0}}$ \\
\hline
\end{tabular}

Classification Summary

Open Question 4.22. Is it the case that $\mathrm{DIM}_{\mathrm{str}}^{\alpha}$ is not in $\Pi_{3}^{0}$ for any $\Delta_{2}^{0}$-computable $\alpha \in$ $[0,1)$ ? 


\section{RESOURCE-BOUNDED DIMENSION AND POLYNOMIAL-TIME REDUCTIONS}

Resource-bounded measure has been used very successfully to study polynomial-time reductions within exponential-time complexity classes. Measure-theoretic arguments were the

first to show that for all $\alpha<1$, every $\leq_{n^{\alpha}-\mathrm{tt}}^{\mathrm{p}}$-hard language for exponential time is exponentially dense [37]. The first plausible hypothesis on NP to separate the $\leq_{\mathrm{m}}^{\mathrm{p}}$ and $\leq_{\mathrm{T}}^{\mathrm{p}}$ reducibilities within NP came from resource-bounded measure [38].

The degrees and spans of languages and classes of languages under polynomial-time reductions have been studied by several researchers using resource-bounded measure.

Definition. Let $\leq_{r}^{\mathrm{p}}$ be a reducibility. For any class $\mathcal{D}$ of languages, let

$$
\mathrm{P}_{r}(\mathcal{D})=\left\{B \subseteq\{0,1\}^{*} \mid(\exists A \in \mathcal{D}) B \leq_{r}^{\mathrm{p}} A\right\}
$$

be the $\leq_{r}^{\mathrm{p}}$-lower span of $\mathcal{D}$ and let

$$
\mathrm{P}_{r}^{-1}(\mathcal{D})=\left\{B \subseteq\{0,1\}^{*} \mid(\exists A \in \mathcal{D}) A \leq_{r}^{\mathrm{p}} B\right\}
$$

be the $\leq_{r}^{\mathrm{p}}$-upper span of $\mathcal{D}$. For any $A \subseteq\{0,1\}^{*}$ we also define $\mathrm{P}_{r}(A)=\mathrm{P}_{r}(\{A\})$ as the $\leq_{r}^{\mathrm{p}}$-lower span of $A, \mathrm{P}_{r}^{-1}(A)=\mathrm{P}_{r}(\{A\})$ as the $\leq_{r}^{\mathrm{p}}$-upper span of $A$, and

$$
\operatorname{deg}_{r}^{\mathrm{p}}(A)=\mathrm{P}_{r}(A) \cap \mathrm{P}_{r}^{-1}(A)
$$

as the $\leq_{r}^{\mathrm{p}}$-degree of $A$.

Also of interest are the classes of hard and complete languages for a given complexity class. 
Definition. For any complexity class $\mathcal{D}$, the class of $\leq_{r}^{\mathrm{p}}$-hard languages for $\mathcal{D}$ is

$$
\mathcal{H}_{r}^{\mathrm{P}}(\mathcal{D})=\left\{A \subseteq\{0,1\}^{*} \mid \mathcal{D} \subseteq \mathrm{P}_{r}(A)\right\}
$$

and the class of $\leq_{r}^{\mathrm{p}}$-complete languages for $\mathcal{D}$ is

$$
\mathcal{C}_{r}^{\mathrm{p}}(\mathcal{D})=\mathcal{D} \cap \mathcal{H}_{r}^{\mathrm{p}}(\mathcal{D})
$$

In this chapter we use resource-bounded dimension to investigate various polynomial-time spans and degrees as well as the hard and complete languages for various complexity classes.

\subsection{Small Spans in Scaled Dimension}

Juedes and Lutz [25] proved the following small span theorem for $\leq_{\mathrm{m}}^{\mathrm{p}}$ reductions in both $\mathrm{E}$ and in EXP.

Theorem 5.1. (Juedes and Lutz [25]) Let $\Delta \in\left\{\mathrm{p}, \mathrm{p}_{2}\right\}$. For every $A \in R(\Delta)$,

$$
\mu\left(\mathrm{P}_{\mathrm{m}}(A) \mid R(\Delta)\right)=0
$$

or

$$
\mu\left(\mathrm{P}_{\mathrm{m}}^{-1}(A) \mid R(\Delta)\right)=\mu_{\Delta}\left(\mathrm{P}_{\mathrm{m}}^{-1}(A)\right)=0
$$

In particular, $\mu\left(\operatorname{deg}_{\mathrm{m}}^{\mathrm{p}}(A) \mid R(\Delta)\right)=0$.

That is, at least one of the upper or lower spans of $A$ is small within $R(\Delta)$. Using a result of Bennett and Gill [6], Juedes and Lutz [25] noted that strengthening Theorem 5.1 from $\leq_{\mathrm{m}}^{\mathrm{p}}$ reductions to $\leq_{\mathrm{T}}^{\mathrm{p}}$ reductions would achieve the separation $\mathrm{BPP} \neq \mathrm{EXP}$. Pursuing this program, small span theorems for reductions of progressively increasing strength between $\leq_{\mathrm{m}}^{\mathrm{p}}$ and $\leq_{\mathrm{T}}^{\mathrm{p}}$ have been obtained by Lindner [29], Ambos-Spies, Neis, and Terwijn [3], and Buhrman and van Melkebeek [10]. 
Recall that resource-bounded dimension is capable of quantitatively distinguishing among the measure 0 sets. With regard to the measure 0 sets in Theorem 5.1, Ambos-Spies, Merkle, Reimann, and Stephan [2] proved the following.

Theorem 5.2. (Ambos-Spies, Merkle, Reimann, and Stephan [2]) For every $A \in \mathrm{E}$,

$$
\operatorname{dim}\left(\operatorname{deg}_{\mathrm{m}}^{\mathrm{p}}(A) \mid \mathrm{E}\right)=\operatorname{dim}\left(\mathrm{P}_{\mathrm{m}}(A) \mid \mathrm{E}\right)
$$

and

$$
\operatorname{dim}_{\mathrm{p}}\left(\operatorname{deg}_{\mathrm{m}}^{\mathrm{p}}(A)\right)=\operatorname{dim}_{\mathrm{p}}\left(\mathrm{P}_{\mathrm{m}}(A)\right)
$$

In particular, as $\operatorname{dim}(\mathrm{E} \mid \mathrm{E})=1$, the $\leq_{\mathrm{m}}^{\mathrm{p}}$-complete degree for $\mathrm{E}$ has dimension 1 within $\mathrm{E}$. This implies that replacing " $\mu$ " by "dim" in Theorem 5.1 makes the statement for E no longer true. In other words, there is no analogue of the small span theorem for dimension in E. Dimension in E cannot distinguish between lower spans and degrees.

In this section we use scaled dimension to investigate polynomial-time spans and degrees and further understand the contrast between Theorems 5.1 and 5.2. We show that the same dichotomy also occurs between the $-3^{\text {rd }}$ and $-2^{\text {nd }}$-orders of scaled dimension. The main contribution is a strengthening of Theorem 5.1 to give a small span theorem for $-3^{\text {rd }}$-order scaled dimension. In contrast, we extend Theorem 5.2 to scaled dimension at orders $i$ with $|i| \leq 2$.

These results hold for scaled dimension in exponential space as well as in exponential time. As an application, we consider the scaled dimension of $\mathcal{C}_{\mathrm{m}}^{\mathrm{p}}(\mathrm{E})$, the class of polynomial-time many-one complete sets for E, within ESPACE. Let $i \in\{-2,-1\}$. We extend a theorem of Lutz [32] and use it to show that determining the $-1^{\text {st }}$ or $-2^{\text {nd }}$-order scaled dimension of $\mathcal{C}_{\mathrm{m}}^{\mathrm{p}}(\mathrm{E})$ in ESPACE would derandomize BPP or separate $\mathrm{P}$ from PSPACE. In contrast, we also show that $\mathcal{C}_{\mathrm{m}}^{\mathrm{p}}(\mathrm{E})$ unconditionally has $-3^{\text {rd }}$-order scaled dimension 0 in ESPACE and $-2^{\text {nd }}$-order scaled dimension 1 in E. 


\subsubsection{Scaled Non-Bi-Immunity and Compressibility}

In this section we introduce some classes involving scales, non-bi-immunity, and compressibility by polynomial-time reductions and calculate their scaled dimensions.

A Turing machine $M$ is consistent with a language $A \subseteq\{0,1\}^{*}$ if for all $x \in\{0,1\}^{*}$,

$$
M(x) \text { halts } \Longleftrightarrow M(x)=A(x)
$$

Let $t$ be a time bound. The fast set of $M$ with respect to $t$ is

$$
F_{M}^{t}=\left\{x \in\{0,1\}^{*} \mid \operatorname{time}_{M}(x) \leq t(|x|)\right\} .
$$

Recall that $A$ is not DTIME(t)-bi-immune if there is a machine $M$ consistent with $A$ such that $F_{M}^{t}$ is infinite.

Definition. For any time bound $t$, let $X(t)$ be the class of all languages that are not DTIME $(t)$ bi-immune.

Let $A \subseteq\{0,1\}^{*}$ and $f:\{0,1\}^{*} \rightarrow\{0,1\}^{*}$. We say that $f$ is a many-one reduction of $A$ if there is some $B \subseteq\{0,1\}^{*}$ such that $x \in A \Longleftrightarrow f(x) \in B$. The collision set of $f$ is

$$
C_{f}=\left\{s_{i} \mid(\exists j<i) f\left(s_{i}\right)=f\left(s_{j}\right)\right\}
$$

Recall that $A$ is compressible by $\leq_{\mathrm{m}}^{\mathrm{DTIME}(t)}$-reductions if there exists an $f \in \operatorname{DTIMEF}(t)$ that is a many-one reduction of $A$ and has $C_{f}$ infinite [25].

Definition. For any time bound $t$, let $C(t)$ be the class of all languages that are compressible by $\leq_{\mathrm{m}}^{\mathrm{DTIME}(t)}$-reductions.

The following theorem asserts that almost every language in $\operatorname{E}$ is $\operatorname{DTIME}\left(2^{c n}\right)$-bi-immune [42] and incompressible by $\leq_{\mathrm{m}}^{\operatorname{DTIME}}\left(2^{c n}\right)$-reductions [25]. 
Theorem 5.3. (Mayordomo [42], Juedes and Lutz [25]) For all $c \in \mathbb{N}$,

$$
\mu_{\mathrm{p}}\left(X\left(2^{c n}\right)\right)=\mu_{\mathrm{p}}\left(C\left(2^{c n}\right)\right)=0
$$

and

$$
\mu_{\mathrm{p}_{2}}\left(X\left(2^{n^{c}}\right)\right)=\mu_{\mathrm{p}_{2}}\left(C\left(2^{n^{c}}\right)\right)=0
$$

The next two definitions introduce scaled versions of $X(t)$ and $C(t)$.

Definition. For any $i \in \mathbb{Z}, \alpha \in[0,1]$, and time bound $t$, let

$$
X_{\alpha}^{(i)}(t)=\left\{\begin{array}{l|l}
A \subseteq\{0,1\}^{*} & \begin{array}{c}
(\exists M) M \text { is consistent with } A \text { and } \\
\left(\exists^{\infty} n\right) \#\left(1, F_{M}^{t}\lceil n) \geq n-g_{i}(n, \alpha)\right.
\end{array}
\end{array}\right\}
$$

That is, $X_{\alpha}^{(i)}(t)$ consists of the languages that are not DTIME $(t)$-bi-immune in a particular strong way: for infinitely many $n$, all but $g_{i}(n, \alpha)$ of the first $n$ strings can be decided in less than $t$ time by a consistent Turing machine.

Definition. For any $i \in \mathbb{Z}, \alpha \in[0,1]$, and time bound $t$, let

$$
C_{\alpha}^{(i)}(t)=\left\{\begin{array}{l|l}
A \in\{0,1\}^{*} & \begin{array}{l}
(\exists f \in \operatorname{DTIMEF}(t)) f \text { is a many-one reduction of } A \\
\text { and }\left(\exists^{\infty} n\right) \#\left(1, C_{f}\lceil n) \geq n-g_{i}(n, \alpha)\right.
\end{array}
\end{array}\right\}
$$

In other words, $C_{\alpha}^{(i)}(t)$ is the class of languages compressible by $\leq_{\mathrm{m}}^{\text {DTIME }(t)}$-reductions where for infinitely many $n$, all but $g_{i}(n, \alpha)$ of the first $n$ strings have collisions under some reduction.

For $\alpha<1, X_{\alpha}^{(i)}\left(2^{n}\right) \subseteq X\left(2^{n}\right)$ and $C_{\alpha}^{(i)}\left(2^{n}\right) \subseteq C\left(2^{n}\right)$, so Theorem 5.3 implies that $X_{\alpha}^{(i)}\left(2^{n}\right)$ and $C_{\alpha}^{(i)}\left(2^{n}\right)$ have measure 0 . We now refine this by calculating their scaled dimensions.

Theorem 5.4. For all $i \in \mathbb{Z}, c \geq 1$, and $\alpha \in[0,1]$,

$$
\operatorname{dim}_{\mathrm{p}}^{(i)}\left(X_{\alpha}^{(i)}\left(2^{c n}\right)\right)=\operatorname{dim}_{\mathrm{p}}^{(i)}\left(C_{\alpha}^{(i)}\left(2^{c n}\right)\right)=\alpha
$$


and

$$
\operatorname{dim}_{\mathrm{p}_{2}}^{(i)}\left(X_{\alpha}^{(i)}\left(2^{n^{c}}\right)\right)=\operatorname{dim}_{\mathrm{p}_{2}}^{(i)}\left(C_{\alpha}^{(i)}\left(2^{n^{c}}\right)\right)=\alpha
$$

Proof. We focus on the p-dimension portion of the theorem; the argument for $\mathrm{p}_{2}$-dimension is identical. If $\alpha=0$, this is trivial, so assume $\alpha \in(0,1]$. Let $s, t>0$ be arbitrary rationals with $s<\alpha<t$. It suffices to show that

$$
s \leq \operatorname{dim}_{\mathrm{p}}^{(i)}\left(X_{\alpha}^{(i)}\left(2^{c n}\right)\right) \leq \operatorname{dim}_{\mathrm{p}}^{(i)}\left(C_{\alpha}^{(i)}\left(2^{c n}\right)\right) \leq t .
$$

The inequality $\operatorname{dim}_{\mathrm{p}}^{(i)}\left(X_{\alpha}^{(i)}\left(2^{c n}\right)\right) \leq \operatorname{dim}_{\mathrm{p}}^{(i)}\left(C_{\alpha}^{(i)}\left(2^{c n}\right)\right)$ holds because of the inclusion $X_{\alpha}^{(i)}\left(2^{c n}\right) \subseteq$ $C_{\alpha}^{(i)}\left(2^{c n}\right)$.

For the lower bound, let $\rho$ be any p-computable measure, say computable in $n^{k}$ time. We define a language $A$ inductively by lengths. Let $s^{\prime} \in(s, \alpha)$ be rational. The first $\left\lfloor g_{i}\left(2^{n}, s^{\prime}\right)\right\rfloor$ bits of $A_{=n}$ are set by diagonalization to minimize $\rho$. The remaining $2^{n}-\left\lfloor g_{i}\left(2^{n}, s^{\prime}\right)\right\rfloor$ bits are identically 0. More formally, if $x$ is the characteristic string of $A_{\leq n-1}$, we choose $v \in\{0,1\}^{\left\lfloor g_{i}\left(2^{n}, s^{\prime}\right)\right\rfloor}$


easily in $X_{s^{\prime}}^{(i)}\left(2^{c n}\right) \subseteq X_{\alpha}^{(i)}\left(2^{c n}\right)$. Let $w \sqsubseteq A$, and let $n$ be such that $2^{n}-1 \leq|w|<2^{n+1}-1$. Then if $|w| \leq 2^{n}-1+\left\lfloor g_{i}\left(2^{n}, s^{\prime}\right)\right\rfloor$, we have

$$
\rho(w) \leq \rho\left(w\left\lceil 2^{n}-1\right) 2^{-\left(|w|-\left(2^{n}-1\right)\right)},\right.
$$

and if $|w| \geq 2^{n}-1+\left\lfloor g_{i}\left(2^{n}, s^{\prime}\right)\right\rfloor$, we have

$$
\begin{aligned}
\rho(w) & \leq \rho\left(w \uparrow\left(2^{n}-1+\left\lfloor g_{i}\left(2^{n}, s^{\prime}\right)\right\rfloor\right)\right) \\
& \leq \frac{\rho\left(w\left\lceil 2^{n}-1\right)\right.}{2^{2^{n}-1+\left\lfloor g_{i}\left(2^{n}, s^{\prime}\right)\right\rfloor-\left(2^{n}-1\right)}} \\
& =\frac{\rho\left(w \uparrow 2^{n}-1\right)}{2^{\left\lfloor g_{i}\left(2^{n}, s^{\prime}\right)\right\rfloor}} .
\end{aligned}
$$


Therefore, in either case,

$$
\begin{aligned}
\log \rho(w)+g_{i}(|w|, s) & \leq \log \rho\left(w \uparrow 2^{n}-1\right)-\left\lfloor g_{i}\left(2^{n}, s^{\prime}\right)\right\rfloor+g_{i}(|w|, s) \\
& \leq \log \rho\left(w \uparrow 2^{n}-1\right)+g_{i}\left(2^{n+1}-1, s\right)-g_{i}\left(2^{n}, s^{\prime}\right)
\end{aligned}
$$

As $g_{i}\left(2^{n}, s^{\prime}\right)-g_{i}\left(2^{n+1}-1, s\right) \rightarrow \infty$ as $n \rightarrow \infty$ since $s<s^{\prime}$, it follows that

$$
\lim _{n \rightarrow \infty} \log \rho\left(A\lceil n)+g_{i}(n, s)=-\infty\right.
$$

Since $\rho$ is an arbitrary p-computable measure, the contrapositive of Lemma 3.41(1) implies that $\operatorname{dim}_{\mathrm{p}}^{(i)}\left(X_{\alpha}^{(i)}\right) \geq s$.

Now we prove the upper bound. Let $A \in C_{\alpha}^{(i)}\left(2^{c n}\right)$ by a function $f \in \operatorname{DTIMEF}\left(2^{c n}\right)$. Define a measure $\rho$ inductively by $\rho(\lambda)=1$ and for all $w \in\{0,1\}^{*}, b \in\{0,1\}$,

1. If $f\left(s_{i}\right) \neq f\left(s_{|w|}\right)$ for all $i<|w|$, then

$$
\rho(w b)=\frac{\rho(w)}{2}
$$

2. Otherwise, let $i=\min \left\{i<|w| \mid f\left(s_{i}\right)=f\left(s_{|w|}\right)\right\}$ and define

$$
\rho(w b)= \begin{cases}\rho(w) & \text { if } b=w[i] \\ 0 & \text { if } b \neq w[i]\end{cases}
$$

Then for all $w \sqsubseteq A$,

$$
\begin{aligned}
\log \rho(w) & =-\#\left(0, C_{f} \uparrow|w|\right) \\
& =\#\left(1, C_{f} \uparrow|w|\right)-|w|
\end{aligned}
$$


Whenever $\#\left(1, C_{f}\lceil n) \geq n-g_{i}(n, \alpha)\right.$, we have

$$
\begin{aligned}
g_{i}(n, t)+\log \rho(A\lceil n) & =g_{i}(n, t)+\#\left(1, C_{f}\lceil n)-n\right. \\
& \geq g_{i}(n, t)-g_{i}(n, \alpha)
\end{aligned}
$$

This happens infinitely often, so

$$
\limsup _{n \rightarrow \infty} g_{i}(n, t)+\log \rho(A\lceil n)=\infty
$$

because $t>\alpha$. Also, $\rho$ is computable in $O\left(|w| \cdot 2^{c \log |w|}\right)=O\left(|w|^{c+1}\right)$ time. Such a $\rho$ can be defined for each $A \in C_{\alpha}^{(i)}\left(2^{c n}\right)$, so $\operatorname{dim}_{\mathrm{p}}^{(i)}\left(C_{\alpha}^{(i)}\left(2^{c n}\right)\right) \leq t$ follows by Theorem 3.45 .

\subsubsection{Small Span Theorem}

In this section we establish our small span theorem for scaled dimension. We begin with a simple, but important, lemma about the scales.

Lemma 5.5. For all $k \geq 1$ and $s, t \in(0,1), g_{3}\left(2^{n^{k}}, s\right)=o\left(g_{2}\left(2^{n}, t\right)\right)$.

Proof. We have

$$
g_{3}\left(2^{n^{k}}, s\right)=2^{2^{\left(\log \log 2^{n^{k}}\right)^{s}}}=2^{2^{(k \log n)^{s}}}
$$

and

$$
g_{2}\left(2^{n}, t\right)=2^{\left(\log 2^{n}\right)^{t}}=2^{n^{t}}=2^{2^{t \log n}}
$$

The lemma holds since $(k \log n)^{s}=o(t \log n)$.

Juedes and Lutz [25] proved that the upper spans of incompressible languages are small. Specifically, for any language $A \in$ EXP that is incompressible by $\leq \mathrm{m}_{\mathrm{m}}^{\mathrm{p}}$-reductions, they showed that $\mu_{\mathrm{P}_{2}}\left(\mathrm{P}_{\mathrm{m}}^{-1}(A)\right)=0$, and if additionally $A \in \mathrm{E}$, then $\mu_{\mathrm{p}}\left(\mathrm{P}_{\mathrm{m}}^{-1}(A)\right)=0$. The following theorem is a scaled dimension analogue of this. For any $i \in \mathbb{Z}$, let

$$
C_{\alpha}^{(i)}(\text { poly })=\bigcup_{c \in \mathbb{N}} C_{\alpha}^{(i)}\left(n^{c}+c\right)
$$


Theorem 5.6. Let $\alpha \in(0,1)$.

1. Let $\Delta \in\{\mathrm{p}$, pspace $\}$. For any $B \in R(\Delta)-C_{\alpha}^{(1)}($ poly $), \operatorname{dim}_{\Delta}^{(-3)}\left(\mathrm{P}_{\mathrm{m}}^{-1}(B)\right)=0$.

2. Let $\Delta \in\left\{\mathrm{p}_{2}, \mathrm{p}_{2}\right.$ space $\}$. For any $B \in R(\Delta)-C_{\alpha}^{(2)}($ poly $), \operatorname{dim}_{\Delta}^{(-3)}\left(\mathrm{P}_{\mathrm{m}}^{-1}(B)\right)=0$.

Proof. We first give the proof for $\Delta=$ p. Let $B \in \mathrm{E}-C_{\alpha}^{(1)}$ (poly) and let $M$ be a Turing machine that decides $B$ in $O\left(2^{c n}\right)$ time. Assume $B \leq_{\mathrm{m}}^{\mathrm{p}} C$ via $f$ where $f$ is computable in $n^{k}$ time almost everywhere. Then for all sufficiently large $n$,

$$
f\left(B_{\leq n}\right) \subseteq C_{\leq n^{k}}
$$

and

$$
\left|f\left(B_{\leq n}\right)\right| \geq g_{1}\left(2^{n+1}-1, \alpha\right) \geq g_{1}\left(2^{n}, \alpha\right)
$$

with the latter holding because $B \notin C_{\alpha}^{(1)}$ (poly).

Let $r \in \mathbb{N}$ such that $\frac{1}{r}<\alpha$. Define $d: \mathbb{N} \rightarrow \mathbb{N}$ by $d(n)=\lfloor n / r\rfloor$. For each $n \in \mathbb{N}$ we define a measure $\rho_{n}:\{0,1\}^{*} \rightarrow[0,1]$ by

$$
\rho_{n}(\lambda)=2^{-n}
$$

and for all $w \in\{0,1\}^{*}$ and $b \in\{0,1\}$,

1. If $|w|<2^{d(n)}$ or $\left[\left(\forall i<2^{n+1}-1\right) f\left(s_{i}\right) \neq f\left(s_{|w|}\right)\right]$, then

$$
\rho_{n}(w b)=\frac{\rho_{n}(w)}{2}
$$

2. Otherwise, let $i=\min \left\{i<2^{n+1}-1 \mid f\left(s_{i}\right)=f\left(s_{|w|}\right)\right\}$ and define

$$
\rho_{n}(w b)= \begin{cases}\rho_{n}(w) & \text { if } b=B[i] \\ 0 & \text { if } b \neq B[i] .\end{cases}
$$

If $|w|<2^{d(n)}$, then $\rho_{n}(w)$ is computable in $O(|w|)$ time. If $|w| \geq 2^{d(n)}$, we can compute $\rho_{n}(w)$ by using $2^{n+1}-1=O\left(|w|^{n / d(n)}\right)=O\left(|w|^{r}\right)$ computations of $M$ and $f$ on strings with length 
at most $n=O(\log |w|)$. Therefore $\rho_{n}(w)$ is computable in $O\left(|w|^{r}\left(2^{c \log |w|}+(\log |w|)^{k}\right)\right)=$ $O\left(|w|^{r+c}\right)$ time for all $w \in\{0,1\}^{*}$.

Let $w_{n}=C \nmid 2^{n^{k}+1}-1$ be the characteristic string of $C_{\leq n^{k}}$. Then letting

$$
m(n)=\left|\left\{j<\left|w_{n}\right| \mid\left(\forall i<2^{n+1}-1\right) f\left(s_{i}\right) \neq f\left(s_{j}\right)\right\}\right|,
$$

we have

$$
\rho_{n}\left(w_{n}\right) \geq \rho_{n}(\lambda) 2^{-2^{d(n)}-m(n)}=2^{-2^{d(n)}-m(n)-n}
$$

By (5.1) and (5.2), we have

$$
m(n) \leq 2^{n^{k}+1}-1-g_{1}\left(2^{n}, \alpha\right)
$$

if $n$ is sufficiently large. In this case,

$$
\log \rho_{n}\left(w_{n}\right) \geq g_{1}\left(2^{n}, \alpha\right)-2^{d(n)}-2^{n^{k}+1}-n
$$

The function $\rho:\{0,1\}^{*} \rightarrow[0, \infty)$ defined by

$$
\rho(w)=\sum_{n=0}^{\infty} \rho_{n}(w)
$$

for all $w$ is a measure by linearity. Notice that $\rho(w)$ can be approximated to a precision of $2^{-l}$ in $O\left(|w|^{r+c} l\right)$ time by adding the first $l+1$ terms of the sum.

Using (5.3), for all sufficiently large $n$, we have

$$
\begin{aligned}
g_{-3}\left(\left|w_{n}\right|, s\right)+\log \rho_{n}\left(w_{n}\right) & =2^{n^{k}+1}+4-g_{3}\left(2^{n^{k}+1}-1,1-s\right)+\log \rho_{n}\left(w_{n}\right) \\
& \geq g_{1}\left(2^{n}, \alpha\right)-g_{3}\left(2^{n^{k}+1}-1,1-s\right)-2^{d(n)}-n .
\end{aligned}
$$

By Lemma 5.5, $g_{3}\left(2^{n^{k}+1}-1,1-s\right)=o\left(g_{1}\left(2^{n}, \alpha\right)\right)$. Also, $2^{d(n)}=2^{\lfloor n / r\rfloor}$ is little-o of $g_{1}\left(2^{n}, \alpha\right)=$ 
$2^{\alpha n}$ because $\alpha>1 / r$. Using these facts, it follows that

$$
\limsup _{n \rightarrow \infty} g_{-3}(n, s)+\log \rho_{n}(C\lceil n)=\infty
$$

Appealing to Theorem 3.45, we establish $\operatorname{dim}_{\mathrm{p}}^{(i)}\left(\mathrm{P}_{\mathrm{m}}^{-1}(B)\right) \leq s$. As $s>0$ is arbitrary, the $\Delta=$ p part of the theorem holds. The argument is identical for $\Delta=$ pspace.

The proof for $\Delta \in\left\{\mathrm{p}_{2}, \mathrm{p}_{2}\right.$ space $\}$ is very similar, so we only sketch the differences for $\Delta=\mathrm{p}_{2}$. Let $B \in \operatorname{EXP}-C_{\alpha}^{(2)}\left(2^{n}\right)$ and let $M$ be a Turing machine that decides $B$ in $O\left(2^{n^{c}}\right)$ time. Assume $B \leq_{\mathrm{m}}^{\mathrm{p}} C$ via $f$. The measures $\rho_{n}$ and $\rho$ are defined in the same way, except we use a different function $d(n)$. For this, we let $r>1 / \alpha$ and define $d(n)=\left\lfloor n^{\epsilon}\right\rfloor$ where $\epsilon=1 / r$. Then, if $|w| \geq 2^{d(n)}$, as before we can compute $\rho_{n}(w)$ by using $2^{n+1}-1$ computations of $M$ and $f$ on strings with length at most $n=O(\log |w|)$. Since $2^{n}=2^{\left(\log 2^{n^{\epsilon}}\right)^{r}}=O\left(2^{(\log |w|)^{r}}\right)$, we can compute $\rho_{n}(w)$ in $O\left(2^{(\log |w|)^{r}} \cdot 2^{(\log |w|)^{c}}\right)=O\left(2^{(\log |w|)^{\max (r, c)}}\right)$ time. Instead of (5.3), we arrive at $\log \rho_{n}\left(w_{n}\right) \geq g_{2}\left(2^{n}, \alpha\right)-2^{d(n)}-2^{n^{k}+1}-n$. The proof is completed in the same way using the fact that $2^{d(n)}=o\left(g_{2}\left(2^{n}, \alpha\right)\right)$ because $\epsilon<\alpha$.

We are now ready to prove our small span theorem.

\section{Theorem 5.7.}

1. Let $\Delta \in\{\mathrm{p}$, pspace $\}$. For every $A \in R(\Delta)$,

$$
\operatorname{dim}^{(1)}\left(\mathrm{P}_{\mathrm{m}}(A) \mid R(\Delta)\right)=0
$$

or

$$
\operatorname{dim}^{(-3)}\left(\mathrm{P}_{\mathrm{m}}^{-1}(A) \mid R(\Delta)\right)=\operatorname{dim}_{\Delta}^{(-3)}\left(\mathrm{P}_{\mathrm{m}}^{-1}(A)\right)=0 .
$$

2. Let $\Delta \in\left\{\mathrm{p}_{2}, \mathrm{p}_{2}\right.$ space $\}$. For every $A \in R(\Delta)$,

$$
\operatorname{dim}^{(2)}\left(\mathrm{P}_{\mathrm{m}}(A) \mid R(\Delta)\right)=0
$$


or

$$
\operatorname{dim}^{(-3)}\left(\mathrm{P}_{\mathrm{m}}^{-1}(A) \mid R(\Delta)\right)=\operatorname{dim}_{\Delta}^{(-3)}\left(\mathrm{P}_{\mathrm{m}}^{-1}(A)\right)=0
$$

Proof. Let $\Delta \in\{\mathrm{p}$, pspace $\}$ and let $A \in R(\Delta)$. We consider two cases.

(I.) Suppose that

$$
\mathrm{P}_{\mathrm{m}}(A) \cap R(\Delta) \subseteq \bigcap_{\alpha \in(0,1)} C_{\alpha}^{(1)}\left(2^{n}\right)
$$

Then $\operatorname{dim}_{\Delta}^{(1)}\left(\mathrm{P}_{\mathrm{m}}(A) \cap R(\Delta)\right) \leq \operatorname{dim}_{\mathrm{p}}^{(1)}\left(C_{\alpha}^{(1)}\left(2^{n}\right)\right) \leq \alpha$ by Theorem 5.4 for all $\alpha \in(0,1)$, so $\operatorname{dim}^{(1)}\left(\mathrm{P}_{\mathrm{m}}(A) \mid R(\Delta)\right)=\operatorname{dim}_{\Delta}^{(1)}\left(\mathrm{P}_{\mathrm{m}}(A) \cap R(\Delta)\right)=0$.

(II.) Otherwise, there is an $\alpha \in(0,1)$ such that

$$
\mathrm{P}_{\mathrm{m}}(A) \cap R(\Delta) \nsubseteq C_{\alpha}^{(1)}\left(2^{n}\right)
$$

Let $B \in \mathrm{P}_{\mathrm{m}}(A) \cap R(\Delta)-C_{\alpha}^{(1)}\left(2^{n}\right)$. Then by Theorem 5.6, $\operatorname{dim}_{\Delta}^{(-3)}\left(\mathrm{P}_{\mathrm{m}}^{-1}(B)\right)=0$. Since $\mathrm{P}_{\mathrm{m}}^{-1}(A) \subseteq \mathrm{P}_{\mathrm{m}}^{-1}(B)$, we have $\operatorname{dim}_{\Delta}^{(-3)}\left(\mathrm{P}_{\mathrm{m}}^{-1}(A)\right)=0$.

Part 2 is proved in the same way.

Theorem 5.7 implies that there is a small span theorem for $-3^{\text {rd }}$-order scaled dimension, but it is stronger than the following.

Corollary 5.8. For every $A \in R(\Delta)$,

$$
\operatorname{dim}^{(-3)}\left(\mathrm{P}_{\mathrm{m}}(A) \mid R(\Delta)\right)=0
$$

or

$$
\operatorname{dim}^{(-3)}\left(\mathrm{P}_{\mathrm{m}}^{-1}(A) \mid R(\Delta)\right)=\operatorname{dim}_{\Delta}^{(-3)}\left(\mathrm{P}_{\mathrm{m}}^{-1}(A)\right)=0
$$

Proof. This follows immediately from Theorem 5.7 using Theorem 3.39.

Theorem 5.1, the small span theorem of Juedes and Lutz [25], is also a corollary of Theorem 5.7. This follows immediately from Lemma 3.38 . 
We also have the following regarding the scaled dimensions of the hard languages for EXP and NP.

Corollary 5.9. 1. $\operatorname{dim}_{\mathrm{p}}^{(-3)}\left(\mathcal{H}_{\mathrm{m}}^{\mathrm{p}}(\mathrm{EXP})\right)=\operatorname{dim}_{\mathrm{p}_{2}}^{(-3)}\left(\mathcal{H}_{\mathrm{m}}^{\mathrm{p}}(\mathrm{EXP})\right)=0$.

2. If $\operatorname{dim}^{(1)}(\mathrm{NP} \mid \mathrm{E})>0$, then $\operatorname{dim}_{\mathrm{p}}^{(-3)}\left(\mathcal{H}_{\mathrm{m}}^{\mathrm{p}}(\mathrm{NP})\right)=0$.

3. If $\operatorname{dim}^{(2)}(\mathrm{NP} \mid \mathrm{EXP})>0$, then $\operatorname{dim}_{\mathrm{P}_{2}}^{(-3)}\left(\mathcal{H}_{\mathrm{m}}^{\mathrm{p}}(\mathrm{NP})\right)=0$.

Proof. Let $H \in \mathcal{C}_{\mathrm{m}}^{\mathrm{p}}(\mathrm{E})$. Then also $H \in \mathcal{C}_{\mathrm{m}}^{\mathrm{p}}(\mathrm{EXP})$, so $\mathrm{P}_{\mathrm{m}}^{-1}(H)=\mathcal{H}_{\mathrm{m}}^{\mathrm{p}}(\mathrm{EXP})$. Since $\operatorname{dim}\left(\mathrm{P}_{\mathrm{m}}(H) \mid\right.$ $\mathrm{E})=\operatorname{dim}_{\mathrm{p}}(\mathrm{E})=1$, Theorem 5.7 tells us that $\operatorname{dim}_{\mathrm{p}}\left(\mathcal{H}_{\mathrm{m}}^{\mathrm{p}}(\mathrm{EXP})\right)=\operatorname{dim}_{\mathrm{p}}\left(\mathrm{P}_{\mathrm{m}}^{-1}(H)\right)=0$.

Parts 2 and 3 follow from Theorem 5.7 using any NP-complete language $A$.

Juedes and Lutz [25] concluded from their small span theorem that every $\leq_{\mathrm{m}}^{\mathrm{p}}$-degree has measure 0 in E and in EXP. From Theorem 5.7 we similarly derive a stronger version of this fact: every $\leq_{\mathrm{m}}^{\mathrm{p}}$-degree actually has $-3^{\mathrm{rd}}$-order dimension 0 .

Corollary 5.10. For every $A \subseteq\{0,1\}^{*}$,

$$
\operatorname{dim}^{(-3)}\left(\operatorname{deg}_{\mathrm{m}}^{\mathrm{p}}(A) \mid R(\Delta)\right)=0
$$

Proof. If $\operatorname{deg}_{\mathrm{m}}^{\mathrm{p}}(A)$ is disjoint from $R(\Delta)$, then $\operatorname{dim}^{(-3)}\left(\operatorname{deg}_{\mathrm{m}}^{\mathrm{p}}(A) \mid R(\Delta)\right)=\operatorname{dim}_{\mathrm{p}}^{(-3)}(\emptyset)=0$, so assume that there is some $B \in \operatorname{deg}_{\mathrm{m}}^{\mathrm{p}}(A) \cap R(\Delta)$. Because $\operatorname{deg}_{\mathrm{m}}^{\mathrm{p}}(A)=\operatorname{deg}_{\mathrm{m}}^{\mathrm{p}}(B)=\mathrm{P}_{\mathrm{m}}(B) \cap$ $\mathrm{P}_{\mathrm{m}}^{-1}(B)$, we have

$$
\operatorname{dim}^{(-3)}\left(\operatorname{deg}_{\mathrm{m}}^{\mathrm{p}}(A) \mid R(\Delta)\right) \leq \operatorname{dim}^{(-3)}\left(\mathrm{P}_{\mathrm{m}}(B) \mid R(\Delta)\right)
$$

and

$$
\operatorname{dim}^{(-3)}\left(\operatorname{deg}_{\mathrm{m}}^{\mathrm{p}}(A) \mid R(\Delta)\right) \leq \operatorname{dim}^{(-3)}\left(\mathrm{P}_{\mathrm{m}}^{-1}(B) \mid R(\Delta)\right)
$$

By Corollary 5.8, we have either $\operatorname{dim}^{(-3)}\left(\mathrm{P}_{\mathrm{m}}(B) \mid R(\Delta)\right)=0$ or $\operatorname{dim}^{(-3)}\left(\mathrm{P}_{\mathrm{m}}^{-1}(B) \mid R(\Delta)\right)=0$. Therefore $\operatorname{dim}^{(-3)}\left(\operatorname{deg}_{\mathrm{m}}^{\mathrm{p}}(A) \mid R(\Delta)\right)=0$. 
The $\leq_{\mathrm{m}}^{\mathrm{p}}$-complete languages for any complexity class has $-3^{\mathrm{rd}}$-order dimension in every $R(\Delta)$

Corollary 5.11. For any class $\mathcal{D}$ of languages, $\operatorname{dim}^{(-3)}\left(\mathcal{C}_{\mathrm{m}}^{\mathrm{p}}(\mathcal{D}) \mid R(\Delta)\right)=0$.

Proof. If $\mathcal{C}_{\mathrm{m}}^{\mathrm{p}}(\mathcal{D})=\emptyset$, this is trivial. Assume $\mathcal{C}_{\mathrm{m}}^{\mathrm{p}}(\mathcal{D}) \neq \emptyset$ and let $A \in \mathcal{C}_{\mathrm{m}}^{\mathrm{p}}(\mathcal{D})$. Then $\mathcal{C}_{\mathrm{m}}^{\mathrm{p}}(\mathcal{D}) \subseteq$ $\operatorname{deg}_{\mathrm{m}}^{\mathrm{p}}(A)$, so this follows from Corollary 5.10.

\subsubsection{Lower Spans vs. Degrees in Orders -2 Through 2}

We now present some results that stand in contrast to the small span theorem of the previous section. We begin by showing that lower spans and degrees have the same scaled dimension in orders $i$ with $|i| \leq 2$.

Theorem 5.12. For any $A \in R(\Delta)$ and $-2 \leq i \leq 2$,

$$
\operatorname{dim}^{(i)}\left(\operatorname{deg}_{\mathrm{m}}^{\mathrm{p}}(A) \mid R(\Delta)\right)=\operatorname{dim}^{(i)}\left(\mathrm{P}_{\mathrm{m}}(A) \mid R(\Delta)\right)
$$

and

$$
\operatorname{dim}_{\Delta}^{(i)}\left(\operatorname{deg}_{\mathrm{m}}^{\mathrm{p}}(A)\right)=\operatorname{dim}_{\Delta}^{(i)}\left(\mathrm{P}_{\mathrm{m}}(A)\right)
$$

Proof. We write the proof for dimension in $R(\mathrm{p})=\mathrm{E}$; the rest of theorem is proved in the same manner.

Let $A \in \mathrm{E}$ be decidable in $O\left(2^{c n}\right)$ time. By monotonicity, $\operatorname{dim}^{(i)}\left(\operatorname{deg}_{\mathrm{m}}^{\mathrm{p}}(A) \mid \mathrm{E}\right) \leq$ $\operatorname{dim}^{(i)}\left(\mathrm{P}_{\mathrm{m}}(A) \mid \mathrm{E}\right)$. For the other inequality, let $t>s>\operatorname{dim}^{(i)}\left(\operatorname{deg}_{\mathrm{m}}^{\mathrm{p}}(A) \mid \mathrm{E}\right)$. By Lemmas 3.41 and 3.42 , for some $l \in \mathbb{N}$ there is an exactly $n^{l}$-time computable measure $\rho$ satisfying

$$
\limsup _{m \rightarrow \infty} g_{i}(m, s)+\log \rho(C \uparrow m)=\infty
$$

for all $C \in \operatorname{deg}_{\mathrm{m}}^{\mathrm{p}}(A) \cap \mathrm{E}$.

Letting $k \geq 1$ be a natural number to be specified later, we define a padding function $f:\{0,1\}^{*} \rightarrow\{0,1\}^{*}$ by

$$
f(x)=0^{|x|^{k}-|x|} x
$$


for all $x$. Let $R=f\left(\{0,1\}^{*}\right)$ be the range of $f$.

Let $B \in \mathrm{P}_{\mathrm{m}}(A)$. We define another language $B^{\prime}$ as

$$
B^{\prime}=(B-R) \cup f(A)
$$

Then $B^{\prime} \in \operatorname{deg}_{\mathrm{m}}^{\mathrm{p}}(A)$. Intuitively, $B^{\prime}$ is a language that is very similar to $B$ but has $A$ encoded sparsely in it. Define a function $\tau:\{0,1\}^{*} \rightarrow\{0,1\}^{*}$ inductively by $\tau(\lambda)=\lambda$ and

$$
\tau(w b)= \begin{cases}\tau(w) b & \text { if } s_{|w|} \notin R \\ \tau(w) 1 & \text { if } s_{|w|} \in R \cap B^{\prime} \\ \tau(w) 0 & \text { if } s_{|w|} \in R-B^{\prime}\end{cases}
$$

for all $w \in\{0,1\}^{*}$ and $b \in\{0,1\}$. Notice that

$$
\tau(B \nmid n)=B^{\prime} \uparrow n
$$

for all $n$.

Define a measure $\gamma$ as follows. For all $w \in\{0,1\}^{*}$ and $b \in\{0,1\}$,

$$
\gamma(w b)= \begin{cases}\frac{\gamma(w)}{2} & \text { if } s_{|w|} \in R \\ \frac{\rho(\tau(w) b)}{\rho(\tau(w))} \gamma(w) & \text { if } s_{|w|} \notin R\end{cases}
$$

Intuitively, $\gamma$ is designed to have performance on $B$ that is similar to $\rho$ 's performance on $B^{\prime}$. This is done by mimicking the conditional probabilities of $\rho$ for strings that are not in $R$. Note that $\gamma(w)$ can be exactly computed in $O\left(|w| \cdot\left(|w|^{l}+2^{(\log |w|)^{c}}\right)\right)=O\left(|w|^{\max (l, c)}\right)$ time. 
Let $n \in N$ and let $2^{(n-1)^{k}+1} \leq m \leq 2^{n^{k}+1}-1$. Then

$$
\begin{aligned}
\log \gamma(B \nmid m) & =\sum_{1 \leq i \leq m} \log \frac{\gamma(B\lceil i)}{\gamma(B\lceil i-1)} \\
& =\sum_{\substack{1 \leq i \leq m \\
s_{i} \notin R}} \log \frac{\rho(\tau(B \nmid i-1) B[i])}{\rho(\tau(B \nmid i-1))}+\sum_{\substack{1 \leq i \leq m \\
s_{i} \in R}} \log \frac{1}{2} \\
& =\sum_{\substack{1 \leq i \leq m \\
s_{i} \notin R}} \log \frac{\rho\left(B^{\prime} \uparrow i\right)}{\rho\left(B^{\prime} \uparrow i-1\right)}-\left|\left\{1 \leq i \leq m \mid s_{i} \in R\right\}\right| \\
& \geq \sum_{1 \leq i \leq m} \log \frac{\rho\left(B^{\prime} \uparrow i\right)}{\rho\left(B^{\prime}\lceil i-1)\right.}-\left|\left\{1 \leq i \leq 2^{n^{k}+1}-1 \mid s_{i} \in R\right\}\right| \\
& =\log \rho\left(B^{\prime}\lceil m)-\sum_{i=0}^{n} 2^{n}\right. \\
& =\log \rho\left(B^{\prime}\lceil m)-2^{n+1}+1 .\right.
\end{aligned}
$$

Now assume that $g_{i}(m, s)+\log \rho\left(B^{\prime} \uparrow m\right) \geq 1$. Then we have $g_{i}(m, t)+\log \gamma(B \nmid m) \geq 1$ if

$$
2^{n+1}+g_{i}(m, s)<g_{i}(m, t)
$$

To establish

$$
\limsup _{n \rightarrow \infty} g_{i}(m, t)+\log \gamma(B \nmid m) \geq 1
$$

it now suffices to show that (5.5) holds for all sufficiently large $m$. For each $-2 \leq i \leq 2$, we now give an appropriate choice of $k$ that yields this.

- $\mathbf{i}=\mathbf{2}$ : Let $k>1 / t$. Then $g_{2}(m, t) \geq g_{2}\left(2^{(n-1)^{k}}, t\right)=2^{(n-1)^{k t}}$, so $2^{n+1}=o\left(g_{2}(m, t)\right)$ because $k t>1$. Also, $g_{2}(m, s)=o\left(g_{2}(m, t)\right)$ since $s<t$, so (5.5) holds when $m$ is sufficiently large.

- $\mathbf{i}=1$ : Let $k=2$. Then $g_{1}(m, t) \geq g_{1}\left(2^{(n-1)^{2}}, t\right)=2^{t(n-1)^{2}}$, so $2^{n+1}=o\left(g_{2}(m, t)\right)$. Also, $g_{1}(m, s)=o\left(g_{1}(m, t)\right)$, so (5.5) holds for sufficiently large $m$.

- $\mathbf{i}=\mathbf{0}$ : Let $k=2$. Then $g_{0}(m, t) \geq g_{0}\left(2^{(n-1)^{2}}, t\right)=t 2^{(n-1)^{2}}$, so $2^{n+1}=o\left(g_{0}(m, t)\right)$. Also, $g_{0}(m, s)=o\left(g_{0}(m, t)\right)$, so (5.5) holds for sufficiently large $m$. 
- $\mathbf{i}=-\mathbf{1}$ : We have $g_{-1}(m, t)=m+1-g_{1}(m, 1-t)$, so $(5.5)$ is true if $2^{n+1}+g_{1}(m, 1-t)<$ $g_{1}(m, 1-s)$. Taking $k=2$, this follows from the argument for $i=1$ above since $1-s>1-t$.

- $\mathbf{i}=-\mathbf{2}$ : Just as in the $i=-1$ case, (5.5) is true if $2^{n+1}+g_{2}(m, 1-t)<g_{2}(m, 1-s)$. Taking $k>1 /(1-s)$, this follows from the argument for $i=2$ above since $1-s>1-t$.

For each $B \in \mathrm{P}_{\mathrm{m}}(A)$, we have given a $O\left(n^{\max (l, c)}\right)$-time computable measure $\gamma$ such that (5.6) holds. By Theorem 3.45, $\operatorname{dim}^{(i)}\left(\mathrm{P}_{\mathrm{m}}(A) \mid \mathrm{E}\right) \leq t$. As $t>\operatorname{dim}^{(i)}\left(\operatorname{deg}_{\mathrm{m}}^{\mathrm{p}}(A) \mid \mathrm{E}\right)$ is arbitrary, this establishes $\operatorname{dim}^{(i)}\left(\mathrm{P}_{\mathrm{m}}(A) \mid \mathrm{E}\right) \leq \operatorname{dim}^{(i)}\left(\operatorname{deg}_{\mathrm{m}}^{\mathrm{p}}(A) \mid \mathrm{E}\right)$.

Theorem 5.12 has as a special case Theorem 5.2 that was proved by Ambos-Spies, Merkle, Reimann, and Stephan [2].

Theorem 5.12 implies that Theorem 5.7 cannot be improved in one respect. For any $i, j \in \mathbb{Z}$, let $\operatorname{SST}[i, j]$ be the assertion that for every $A \in \mathrm{E}$, either

$$
\operatorname{dim}^{(i)}\left(\mathrm{P}_{\mathrm{m}}(A) \mid \mathrm{E}\right)=0
$$

or

$$
\operatorname{dim}^{(j)}\left(\mathrm{P}_{\mathrm{m}}^{-1}(A) \mid \mathrm{E}\right)=0 .
$$

Let $H \in \mathcal{C}_{\mathrm{m}}^{\mathrm{p}}(\mathrm{E})$. Then

$$
\operatorname{dim}^{(-2)}\left(\mathrm{P}_{\mathrm{m}}(H) \mid \mathrm{E}\right)=\operatorname{dim}^{(-2)}(\mathrm{E} \mid \mathrm{E})=1,
$$

so $\operatorname{dim}^{(-2)}\left(\operatorname{deg}_{\mathrm{m}}^{\mathrm{p}}(H) \mid \mathrm{E}\right)=1$ by Theorem 5.12 , which in turn implies

$$
\operatorname{dim}^{(-2)}\left(\mathrm{P}_{\mathrm{m}}^{-1}(H) \mid \mathrm{E}\right)=1
$$

Therefore, $\operatorname{SST}[i, j]$ is true only if $i \leq-3$ or $j \leq-3$. Theorem 5.7 asserts $\operatorname{SST}[1,-3]$, so the -3 in it cannot be improved to -2 . 
We have the following corollary regarding the classes of complete sets for E, EXP, and NP.

Corollary 5.13. Let $-2 \leq i \leq 2$.

1. $\operatorname{dim}^{(i)}\left(\mathcal{C}_{\mathrm{m}}^{\mathrm{p}}(\mathrm{E}) \mid \mathrm{E}\right)=\operatorname{dim}^{(i)}\left(\mathcal{C}_{\mathrm{m}}^{\mathrm{p}}(\mathrm{EXP}) \mid \mathrm{EXP}\right)=1$

2. $\operatorname{dim}^{(i)}(\mathrm{NP} \mid \mathrm{E})=\operatorname{dim}^{(i)}\left(\mathcal{C}_{\mathrm{m}}^{\mathrm{p}}(\mathrm{NP}) \mid \mathrm{E}\right)$.

3. $\operatorname{dim}^{(i)}(\mathrm{NP} \mid \mathrm{EXP})=\operatorname{dim}^{(i)}\left(\mathcal{C}_{\mathrm{m}}^{\mathrm{p}}(\mathrm{NP}) \mid \mathrm{EXP}\right)$

Proof. Let $H \in \mathcal{C}_{\mathrm{m}}^{\mathrm{p}}(\mathrm{E})$. Then $\mathcal{C}_{\mathrm{m}}^{\mathrm{p}}(\mathrm{E})=\operatorname{deg}_{\mathrm{m}}^{\mathrm{p}}(H) \cap \mathrm{E}$, so $\operatorname{dim}^{(i)}\left(\mathcal{C}_{\mathrm{m}}^{\mathrm{p}}(\mathrm{E}) \mid \mathrm{E}\right)=\operatorname{dim}^{(i)}\left(\operatorname{deg}_{\mathrm{m}}^{\mathrm{p}}(H) \mid\right.$ $\mathrm{E})=\operatorname{dim}^{(i)}\left(\mathrm{P}_{\mathrm{m}}(H) \mid \mathrm{E}\right)=\operatorname{dim}_{\mathrm{p}}^{(i)}(\mathrm{E})=1$ by Theorem 5.12. The other statements follow similarly.

We can now observe a difference between the $-3^{\text {rd }}$ and $-2^{\text {nd }}$-order scaled dimensions regarding complete degrees. Corollaries 5.11 and 5.13 together with Theorem 3.39 tell us that for $\mathcal{D} \in\{\mathrm{E}, \mathrm{EXP}\}$,

$$
\operatorname{dim}^{(i)}\left(\mathcal{C}_{\mathrm{m}}^{\mathrm{p}}(\mathcal{D}) \mid \mathcal{D}\right)= \begin{cases}0 & \text { if } i \leq-3 \\ 1 & \text { if } i \geq-2\end{cases}
$$

and

$$
\operatorname{dim}^{(i)}\left(\mathcal{C}_{\mathrm{m}}^{\mathrm{p}}(\mathrm{NP}) \mid \mathcal{D}\right)= \begin{cases}0 & \text { if } i \leq-3 \\ \operatorname{dim}^{(i)}(\mathrm{NP} \mid \mathcal{D}) & \text { if } i \geq-2 .\end{cases}
$$

In Section 5.1.5 we will discuss the scaled dimension of $\mathcal{C}_{\mathrm{m}}^{\mathrm{p}}(\mathrm{E})$ within ESPACE. The following extension of Theorem 5.12 will be useful.

Theorem 5.14. For all $-2 \leq i \leq 2$,

$$
\operatorname{dim}^{(i)}\left(\mathcal{C}_{\mathrm{m}}^{\mathrm{p}}(\mathrm{E}) \mid \mathrm{ESPACE}\right)=\operatorname{dim}^{(i)}(\mathrm{E} \mid \mathrm{ESPACE})
$$

Proof. We use the construction from the proof of Theorem 5.12. Let $t>s>\operatorname{dim}^{(i)}\left(\mathcal{C}_{\mathrm{m}}^{\mathrm{p}}(\mathrm{E}) \mid\right.$ ESPACE) and take an exactly $n^{l}$-space computable measure $\rho$ satisfying (5.4) for all $C \in$ 
$\mathcal{C}_{\mathrm{m}}^{\mathrm{p}}(\mathrm{E})$. Fix an $A \in \mathcal{C}_{\mathrm{m}}^{\mathrm{p}}(\mathrm{E})$. For any $B \in \mathrm{E}$, the set $B^{\prime}$ constructed from $A$ and $B$ is in $\mathcal{C}_{\mathrm{m}}^{\mathrm{p}}(\mathrm{E})$. The arguments then show $\operatorname{dim}^{(i)}(\mathrm{E} \mid \mathrm{ESPACE}) \leq t$.

\subsection{4 $\leq_{1-\mathrm{tt}}^{\mathrm{P}}$-Lower Spans vs. $\leq_{\mathrm{m}}^{\mathrm{p}}$-Lower Spans}

Theorem 5.12 is also true for most other polynomial-time reducibilities. (This fact was mentioned in [2] for Theorem 5.2 when it was proved.) To replace $\leq_{\mathrm{m}}^{\mathrm{p}}$ by $\leq_{r}^{\mathrm{p}}$ in the theorem, we only need to have $B^{\prime} \in \operatorname{deg}_{r}^{\mathrm{p}}(A)$ for the set $B^{\prime}$ that was constructed in the proof from $B \in \mathrm{P}_{r}(A)$. In particular, Theorem 5.12 is true for the $\leq_{1-\mathrm{tt}}^{\mathrm{P}}$ reducibility. In this section we show that this holds because of another reason: the scaled dimensions of $\leq_{1-\mathrm{tt}^{\mathrm{P}}}$-lower spans and $\leq_{\mathrm{m}}^{\mathrm{p}}$-lower spans are always the same.

The following proposition was used to show that a set is weakly $\leq_{\mathrm{m}}^{\mathrm{p}}$-complete for exponential time if and only if it is $\leq_{1-\mathrm{tt}^{-}}$-complete.

Proposition 5.15. (Ambos-Spies, Mayordomo, and Zheng [1]) Let $A \leq_{1-\mathrm{tt}}^{\mathrm{P}} B$. Then there is a language $C \in \mathrm{P}$ such that

$$
\hat{A}=(A \cap C) \cup\left(A^{c} \cap C^{c}\right) \leq_{\mathrm{m}}^{\mathrm{p}} B
$$

The idea of the following lemma also comes from [1].

Lemma 5.16. Let $i \in \mathbb{Z}$. Let $\mathcal{C}, \hat{\mathcal{C}}$ be classes of languages such that for any $A \in \mathcal{C}$, there is

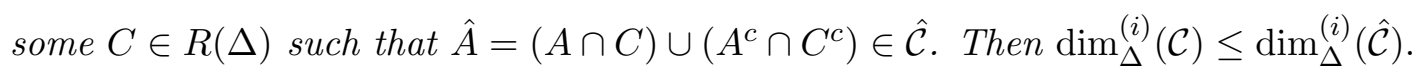

Proof. We prove this for $\Delta=\mathrm{p}$. The other cases are proved by identical arguments.

Let $s>\operatorname{dim}_{\mathrm{p}}^{(i)}(\hat{\mathcal{C}})$ be rational and obtain $\rho$ computable in $O\left(n^{r}\right)$ time from Lemma 3.41 such that

$$
\limsup _{n \rightarrow \infty} g_{i}(n, s)+\log \rho(\hat{A}\lceil n)=\infty
$$

for all $\hat{A} \in \hat{\mathcal{C}}$.

Let $A \in \mathcal{C}$ and let $C \in \operatorname{DTIME}\left(n^{k}\right)$ such that $\hat{A}=(A \cap C) \cup\left(A^{c} \cap C^{c}\right) \in \hat{\mathcal{C}}$. Define a 
function $\tau:\{0,1\}^{*} \rightarrow\{0,1\}^{*}$ by

$$
\tau(w)[j]= \begin{cases}w[j] & \text { if } s_{j} \in C \\ 1-w[j] & \text { if } s_{j} \notin C\end{cases}
$$

for each $0 \leq j<|w|$. Define another measure $\rho^{\prime}$ by

$$
\rho^{\prime}(w)=\rho(\tau(w))
$$

Then for all $n$,

$$
\rho^{\prime}(A\lceil n)=\rho(\tau(A\lceil n))=\rho(\hat{A}\lceil n) .
$$

Therefore

$$
\limsup _{n \rightarrow \infty} g_{i}(n, s)+\log \rho^{\prime}(A\lceil n)=\infty
$$

because of (5.7). As $\rho^{\prime}$ is computable in time $O\left(|w| \cdot(\log |w|)^{k}+|w|^{r}\right)=O\left(|w|^{2}+|w|^{r}\right)$, it follows by Theorem 3.45 that $\operatorname{dim}_{\mathrm{p}}^{(i)}(\mathcal{C}) \leq s$.

We now show that the scaled dimension of $\mathrm{a} \leq_{\mathrm{m}}^{\mathrm{p}}$-lower span is always equal to the scaled dimension of the $\leq_{1-\mathrm{tt}}^{\mathrm{P}}$-lower span.

Theorem 5.17. Let $\mathcal{D}$ be a class of languages and let $i \in \mathbb{Z}$. Then

$$
\operatorname{dim}_{\Delta}^{(i)}\left(\mathrm{P}_{\mathrm{m}}(\mathcal{D})\right)=\operatorname{dim}_{\Delta}^{(i)}\left(\mathrm{P}_{1-\mathrm{tt}}(\mathcal{D})\right)
$$

and

$$
\operatorname{dim}^{(i)}\left(\mathrm{P}_{\mathrm{m}}(\mathcal{D}) \mid R(\Delta)\right)=\operatorname{dim}^{(i)}\left(\mathrm{P}_{1-\mathrm{tt}}(\mathcal{D}) \mid R(\Delta)\right)
$$

Proof. By Proposition 5.15, for each $A \in \mathrm{P}_{1-\mathrm{tt}}(\mathcal{D})$ there is a language $C \in \mathrm{P}$ such that $\hat{A}=(A \cap C) \cup\left(A^{c} \cap C^{c}\right) \in \mathrm{P}_{\mathrm{m}}(\mathcal{D})$. Let $\hat{\mathcal{C}}$ be the set of all such $\hat{A}$ as $A$ ranges over $\mathrm{P}_{1-\mathrm{tt}}(\mathcal{D})$. Then by Lemma 5.16 ,

$$
\operatorname{dim}_{\Delta}^{(i)}\left(\mathrm{P}_{1-\mathrm{tt}}(\mathcal{D})\right) \leq \operatorname{dim}_{\Delta}^{(i)}(\hat{\mathcal{C}})
$$


As $\hat{\mathcal{C}} \subseteq \mathrm{P}_{\mathrm{m}}(\mathcal{D}) \subseteq \mathrm{P}_{1-\mathrm{tt}}(\mathcal{D})$, we also have

$$
\operatorname{dim}_{\Delta}^{(i)}(\hat{\mathcal{C}}) \leq \operatorname{dim}_{\Delta}^{(i)}\left(\mathrm{P}_{\mathrm{m}}(\mathcal{D})\right) \leq \operatorname{dim}_{\Delta}^{(i)}\left(\mathrm{P}_{1-\mathrm{tt}}(\mathcal{D})\right)
$$

so the first equality holds. The proof for dimension in $R(\Delta)$ is analogous.

We can now give a stronger version of Theorem 5.12.

Corollary 5.18. For any $A \in R(\Delta)$ and $-2 \leq i \leq 2$,

$$
\begin{aligned}
\operatorname{dim}^{(i)}\left(\mathrm{P}_{\mathrm{m}}(A) \mid R(\Delta)\right) & =\operatorname{dim}^{(i)}\left(\operatorname{deg}_{\mathrm{m}}^{\mathrm{p}}(A) \mid R(\Delta)\right) \\
\operatorname{dim}^{(i)}\left(\mathrm{P}_{1-\mathrm{tt}}(A) \mid R(\Delta)\right) & =\operatorname{dim}^{(i)}\left(\operatorname{deg}_{1-\mathrm{tt}}^{\mathrm{p}}(A) \mid R(\Delta)\right),
\end{aligned}
$$

and similarly with $\operatorname{dim}^{(i)}(\cdot \mid R(\Delta))$ replaced by $\operatorname{dim}_{\Delta}^{(i)}(\cdot)$.

Proof. From Theorems 5.12 and 5.17 we have

$$
\operatorname{dim}^{(i)}\left(\operatorname{deg}_{\mathrm{m}}^{\mathrm{p}}(A) \mid R(\Delta)\right)=\operatorname{dim}^{(i)}\left(\mathrm{P}_{\mathrm{m}}(A) \mid R(\Delta)\right)=\operatorname{dim}^{(i)}\left(\mathrm{P}_{1-\mathrm{tt}}(A) \mid R(\Delta)\right) .
$$

By monotonicity, we have

$$
\operatorname{dim}^{(i)}\left(\operatorname{deg}_{\mathrm{m}}^{\mathrm{p}}(A) \mid R(\Delta)\right) \leq \operatorname{dim}^{(i)}\left(\operatorname{deg}_{1-\mathrm{tt}}^{\mathrm{p}}(A) \mid R(\Delta)\right) \leq \operatorname{dim}^{(i)}\left(\mathrm{P}_{1-\mathrm{tt}}(A) \mid R(\Delta)\right)
$$

so the equalities displayed in the statement of the corollary are true. The proof for $\operatorname{dim}_{\Delta}^{(i)}(\cdot)$ is analogous.

Theorem 5.17 also yields a strengthening of Theorem 5.7: the $\mathrm{P}_{\mathrm{m}}(A)$ in it can be replaced by $\mathrm{P}_{1-\mathrm{tt}}(A)$. In fact, it is also possible to replace the $\mathrm{P}_{\mathrm{m}}^{-1}(A)$ in Theorem 5.7 by $\mathrm{P}_{1-\mathrm{tt}}^{-1}(A)$ by extending Theorems 5.4 and 5.6 to deal with $\leq_{1-\mathrm{tt}^{\mathrm{P}}}^{\mathrm{P}}$-reductions. We omit the details. 


\subsubsection{The Scaled Dimension of $\mathcal{C}_{\mathrm{m}}^{\mathrm{p}}(\mathrm{E})$ in ESPACE}

Lutz [34] proved a small span theorem for nonuniform Turing reductions in ESPACE. This implies that $\mathcal{C}_{\mathrm{m}}^{\mathrm{p}}(\mathrm{E})$ has measure 0 in ESPACE. In Corollary 5.11 we saw that $\mathcal{C}_{\mathrm{m}}^{\mathrm{p}}(\mathrm{E})$ actually has $-3^{\text {rd }}$-order scaled dimension 0 in ESPACE. In this section we show that determining the $-2^{\text {nd }}$ - or $-1^{\text {st }}$-order scaled dimension of $\mathcal{C}_{\mathrm{m}}^{\mathrm{p}}(\mathrm{E})$ in ESPACE would yield a proof of $\mathrm{P}=\mathrm{BPP}$ or $\mathrm{P} \neq \mathrm{PSPACE}$.

The $\mathrm{P}=\mathrm{BPP}$ hypothesis was related to the measure of $\mathrm{E}$ in ESPACE by Lutz [32].

Theorem 5.19. (Lutz [32]) If $\mu(\mathrm{E} \mid \mathrm{ESPACE}) \neq 0$, then $\mathrm{P}=\mathrm{BPP}$.

We will extend this result to scaled dimension. We now recall the tools Lutz used to prove it.

Nisan and Wigderson [45] showed that BPP can be derandomized if there is a decision problem in E that requires exponential-size circuits to approximately solve. The hardness of a decision problem at a given length is the minimum size of a circuit that can approximately solve it. The details of the definition of this hardness are not needed in this paper; we only need to recall existing results regarding classes of languages with exponential hardness.

Definition. Let $H_{\alpha}$ be the class of all languages that have hardness at least $2^{\text {an }}$ almost everywhere.

The aforementioned derandomization of BPP can be stated as follows.

Theorem 5.20. (Nisan and Wigderson [45]) If there is an $\mathrm{E} \cap H_{\alpha} \neq \emptyset$ for some $\alpha>0$, then $\mathrm{P}=\mathrm{BPP}$

We will also need space-bounded Kolmogorov complexity.

Definition. Given a machine $M$, a space bound $s: \mathbb{N} \rightarrow \mathbb{N}$, a language $L \subseteq\{0,1\}^{*}$, and a natural number $n$, the $s$-space-bounded Kolmogorov complexity of $L_{=n}$ relative to $M$ is

$$
\operatorname{KS}_{M}^{s}\left(L_{=n}\right)=\min \left\{|\pi| \mid M(\pi, n)=\chi_{L_{=n}} \text { in } \leq s\left(2^{n}\right) \text { space }\right\}
$$

i.e., the length of the shortest program $\pi$ such that $M$, on input $(\pi, n)$, outputs the characteristic string of $L_{=n}$ and halts without using more than $s\left(2^{n}\right)$ workspace. 
Well-known simulation techniques show that there exists a machine $U$ which is optimal in the sense that for each machine $M$ there is a constant $c$ such that for all $s, L$ and $n$ we have

$$
\operatorname{KS}_{U}^{c s+c}\left(L_{=n}\right) \leq \operatorname{KS}_{M}^{s}\left(L_{=n}\right)+c .
$$

As usual, we fix such a universal machine and omit it from the notation.

Definition. For each space bound $s: \mathbb{N} \rightarrow \mathbb{N}$ and function $f: \mathbb{N} \rightarrow \mathbb{N}$ define the complexity class

$$
\mathrm{KS}^{s}(f)=\left\{L \subseteq\{0,1\}^{*} \mid\left(\forall^{\infty} n\right) \mathrm{KS}^{s}\left(L_{=n}\right)<f(n)\right\}
$$

Lutz showed that $H_{\alpha}$ has measure 1 in ESPACE (i.e., that $H_{\alpha}^{c}$ has measure 0 in ESPACE) if $\alpha<1 / 3$ by showing that languages not in $H_{\alpha}$ have low space-bounded Kolmogorov complexity.

Lemma 5.21. (Lutz [32]) There exist a polynomial $q$ and a constant $c$ such that for all $0<\alpha<\beta<1$,

$$
H_{\alpha}^{c} \subseteq \mathrm{KS}_{\text {i.o. }}^{q}\left(2^{n}-c 2^{(1-2 \alpha) n}+2^{\beta n}\right) .
$$

The class on the right in Lemma 5.21 has measure 0 in ESPACE [33]. The scaled dimensions of similar space-bounded Kolmogorov complexity classes were studied in [23].

Theorem 5.22. (Hitchcock, Lutz, and Mayordomo [23]) For any $i \leq-1$, polynomial $q(n)=$ $\Omega\left(n^{2}\right)$, and $\alpha \in[0,1]$,

$$
\operatorname{dim}^{(i)}\left(\operatorname{KS}_{\text {i.o. }}^{q}\left(g_{i}\left(2^{n}, \alpha\right)\right) \mid \mathrm{ESPACE}\right)=\alpha
$$

Lemma 5.21 and Theorem 5.22 provide an easy upper bound on the $-1^{\text {st }}$-order scaled dimension of $H_{\alpha}^{c}$ in ESPACE.

Corollary 5.23. If $0<\alpha<1 / 3$, then

$$
\operatorname{dim}_{\text {pspace }}^{(-1)}\left(H_{\alpha}^{c}\right) \leq 2 \alpha .
$$


Proof. Let $\epsilon>0$ and $\beta \in(\alpha, 1-2 \alpha)$. Then for all sufficiently large $n$,

$$
\begin{aligned}
2^{n}-c 2^{(1-2 \alpha) n}+2^{\beta n} & <2^{n}+1-2^{(1-2 \alpha-\epsilon) n} \\
& =g_{1}\left(2^{n}, 2 \alpha+\epsilon\right)
\end{aligned}
$$

so Lemma 5.21 implies $H_{\alpha}^{c} \subseteq \mathrm{KS}_{\text {i.o. }}^{q}\left(g_{1}\left(2^{n}, 2 \alpha+\epsilon\right)\right)$. Therefore $\operatorname{dim}^{(-1)}\left(H_{\alpha}^{c} \mid \mathrm{ESPACE}\right) \leq 2 \alpha+\epsilon$ by Theorem 5.22 .

We can now state a stronger version of Theorem 5.19. The hypothesis has been weakened, but the conclusion remains the same.

Theorem 5.24. If $\operatorname{dim}^{(-1)}(\mathrm{E} \mid \mathrm{ESPACE})>0$, then $\mathrm{P}=\mathrm{BPP}$.

Proof. Assume the hypothesis and let $s=\min \left\{1 / 2, \operatorname{dim}^{(-1)}(\mathrm{E} \mid \mathrm{ESPACE})\right\}$. Then by Corollary 5.23, $\mathrm{E} \nsubseteq H_{s / 2}^{c}$, i.e., $\mathrm{E} \cap H_{s / 2} \neq \emptyset$. Therefore $\mathrm{P}=\mathrm{BPP}$ by Theorem 5.20.

We now relate the scaled dimension of $\mathcal{C}_{\mathrm{m}}^{\mathrm{p}}(\mathrm{E})$ to the $\mathrm{P} \stackrel{?}{=} \mathrm{PSPACE}$ and $\mathrm{P} \stackrel{?}{=} \mathrm{BPP}$ problems.

Theorem 5.25. For $i \in\{-2,-1\}$,

$$
\operatorname{dim}^{(i)}\left(\mathcal{C}_{\mathrm{m}}^{\mathrm{p}}(\mathrm{E}) \mid \mathrm{ESPACE}\right)<1 \Rightarrow \mathrm{P} \neq \mathrm{PSPACE}
$$

and

$$
\operatorname{dim}^{(i)}\left(\mathcal{C}_{\mathrm{m}}^{\mathrm{p}}(\mathrm{E}) \mid \mathrm{ESPACE}\right)>0 \Rightarrow \mathrm{P}=\mathrm{BPP}
$$

Proof. From Theorem 5.14 we know that $\operatorname{dim}^{(i)}\left(\mathcal{C}_{\mathrm{m}}^{\mathrm{p}}(\mathrm{E}) \mid \mathrm{ESPACE}\right)=\operatorname{dim}^{(i)}(\mathrm{E} \mid \mathrm{ESPACE})$. Also, $\operatorname{dim}^{(i)}(\mathrm{E} \mid$ ESPACE $)<1$ implies $\mathrm{E} \neq$ ESPACE which implies $\mathrm{P} \neq \mathrm{PSPACE}$ [7]. This proves the first implication. The second one follows from Theorem 5.24 since $\operatorname{dim}^{(i)}\left(\mathcal{C}_{\mathrm{m}}^{\mathrm{p}}(\mathrm{E}) \mid\right.$ ESPACE $)>0$ implies $\operatorname{dim}^{(-1)}(\mathrm{E} \mid \mathrm{ESPACE})>0$.

In other words, establishing any nontrivial upper or lower bound on $\operatorname{dim}^{(-1)}\left(\mathcal{C}_{\mathrm{m}}^{\mathrm{p}}(\mathrm{E})\right.$ ESPACE) or $\operatorname{dim}^{(-2)}\left(\mathcal{C}_{\mathrm{m}}^{\mathrm{p}}(\mathrm{E}) \mid \mathrm{ESPACE}\right)$ would derandomize BPP or separate $\mathrm{P}$ from PSPACE. 
This is in contrast to the unconditional facts from Corollaries 5.10 and 5.13 that

$$
\operatorname{dim}^{(-3)}\left(\mathcal{C}_{\mathrm{m}}^{\mathrm{p}}(\mathrm{E}) \mid \mathrm{ESPACE}\right)=0
$$

and

$$
\operatorname{dim}^{(-2)}\left(\mathcal{C}_{\mathrm{m}}^{\mathrm{p}}(\mathrm{E}) \mid \mathrm{E}\right)=\operatorname{dim}^{(-1)}\left(\mathcal{C}_{\mathrm{m}}^{\mathrm{p}}(\mathrm{E}) \mid \mathrm{E}\right)=1
$$

\subsection{Degrees of Arbitrary Dimensions}

Ambos-Spies, Merkle, Reimann, and Stephan [2] proved the following result asserting the existence of degrees with arbitrary dimensions in exponential time.

Theorem 5.26. (Ambos-Spies, Merkle, Reimann, and Stephan [2]) For any $\Delta_{2}^{0}$-computable real number $x \in[0,1]$ there exists $A \in \mathrm{E}$ such that

$$
\operatorname{dim}_{\mathrm{p}}\left(\operatorname{deg}_{\mathrm{m}}^{\mathrm{p}}(A)\right)=\operatorname{dim}\left(\operatorname{deg}_{\mathrm{m}}^{\mathrm{p}}(A) \mid \mathrm{E}\right)=x
$$

In this section we develop a proof of the following extension of Theorem 5.26.

Theorem 5.27. For every pair of $\Delta_{2}^{0}$-computable real numbers $x, y$ with $0 \leq x \leq y \leq 1$, there exists $A \in \mathrm{E}$ such that

$$
\operatorname{dim}_{\mathrm{p}}\left(\operatorname{deg}_{\mathrm{m}}^{\mathrm{p}}(A)\right)=\operatorname{dim}\left(\operatorname{deg}_{\mathrm{m}}^{\mathrm{p}}(A) \mid \mathrm{E}\right)=x
$$

and

$$
\operatorname{Dim}_{\mathrm{p}}\left(\operatorname{deg}_{\mathrm{m}}^{\mathrm{p}}(A)\right)=\operatorname{Dim}\left(\operatorname{deg}_{\mathrm{m}}^{\mathrm{p}}(A) \mid \mathrm{E}\right)=y
$$

The proof of Theorem 5.27 is motivated by the analogous, but simpler, arguments used by Ambos-Spies, Merkle, Reimann and Stephan [2] to prove Theorem 5.26. Like most dimension calculations, our proof consists of separate lower and upper bound arguments. The results 
from here through Lemma 5.34 are used for the lower bound. Lemma 5.35 uses Theorem 3.33 to establish the upper bound. The proof of Theorem 5.27 follows Lemma 5.35.

The following result is an exact dual of Theorem 5.2 and has an analogous proof.

Theorem 5.28. For any $A \in \mathrm{E}$,

$$
\operatorname{Dim}_{\mathrm{p}}\left(\operatorname{deg}_{\mathrm{m}}^{\mathrm{p}}(A)\right)=\operatorname{Dim}_{\mathrm{p}}\left(\mathrm{P}_{\mathrm{m}}(A)\right)
$$

and

$$
\operatorname{Dim}\left(\operatorname{deg}_{\mathrm{m}}^{\mathrm{p}}(A) \mid \mathrm{E}\right)=\operatorname{Dim}\left(\mathrm{P}_{\mathrm{m}}(A) \mid \mathrm{E}\right)
$$

We will use randomness relative to a bias sequence to prove Theorem 5.27. A bias is a real number $\beta \in[0,1]$. Intuitively, if we toss a $0 / 1$-valued coin with bias $\beta$, then $\beta$ is the probability of the outcome 1 . A bias sequence is a sequence $\vec{\beta}=\left(\beta_{0}, \beta_{1}, \beta_{2}, \ldots\right)$ of biases. If $\vec{\beta}$ is a bias sequence, then the $\vec{\beta}$-coin-toss probability measure is the probability $\mu^{\vec{\beta}}$ on $\mathbf{C}$ defined by

$$
\mu^{\vec{\beta}}(w)=\prod_{i=0}^{|w|-1} \beta_{i}(w)
$$

where $\beta_{i}(w)=\left(2 \beta_{i}-1\right) w[i]+\left(1-\beta_{i}\right)$, i.e., $\beta_{i}(w)=$ if $w[i]$ then $\beta_{i}$ else $1-\beta_{i}$. That is, $\mu^{\vec{\beta}}$ is the probability that $S \in \mathbf{C}_{w}$ when $S \in \mathbf{C}$ is chosen according to a random experiment in which for each $i$, independently of all other $j$, the $i^{\text {th }}$ bit of $S$ is decided by tossing a 0/1valued coin whose probability of 1 is $\beta_{i}$. In the case where the biases $\beta_{i}$ are all the same, i.e., $\vec{\beta}=(\beta, \beta, \beta, \ldots)$ for some $\beta \in[0,1]$, we write $\mu^{\beta}$ for $\mu^{\vec{\beta}}$, and (5.1) simplifies to

$$
\mu^{\beta}(w)=(1-\beta)^{\#(0, w)} \beta^{\#(1, w)},
$$

where $\#(b, w)$ is the number of times the bit $b$ appears in the string $w$. The uniform probability measure on $\mathbf{C}$ is the probability measure $\mu=\mu^{\frac{1}{2}}$, for which (5.2) simplifies to

$$
\mu(w)=2^{-|w|}
$$


for all $w \in\{0,1\}^{*}$.

Definition. Let $\nu$ be a probability measure on $\mathbf{C}$. A $\nu$-martingale is a function $d:\{0,1\}^{*} \rightarrow$ $[0, \infty)$ that satisfies the condition

$$
d(w) \nu(w)=d(w 0) \nu(w 0)+d(w 1) \nu(w 1)
$$

for all $w \in\{0,1\}^{*}$.

Note that a $\mu$-martingale is a martingale. If $\vec{\beta}$ is a bias sequence, then we call a $\mu^{\vec{\beta}_{-}}$ martingale simply a $\vec{\beta}$-martingale.

We will use resource-bounded notions of randomness that have been investigated by Schnorr [53], Lutz [33], Ambos-Spies, Terwijn, and Zheng [4], and others.

Definition. Let $\nu$ be a probability measure on $\mathbf{C}$, and let $t: \mathbb{N} \rightarrow \mathbb{N}$.

1. A sequence $R \in \mathbf{C}$ is $\Delta$ - $\nu$-random, and we write $\mathbb{R} \in \operatorname{RAND}^{\nu}(\Delta)$, if there is no $\Delta$ computable $\nu$-martingale that succeeds on $R$.

2. A sequence $R \in \mathbf{C}$ is $t(n)-\nu$-random, and we write $R \in \operatorname{RAND}^{\nu}(t(n))$, if there is no $O(t(n))$-time-computable $\nu$-martingale that succeeds on $R$.

We write $\operatorname{RAND}^{\vec{\beta}}(t(n))$ for $\operatorname{RAND}^{\mu^{\vec{\beta}}}(t(n))$. We also say $\mu_{t(n)}^{\vec{\beta}}(X)=0$ if there is a $O(t(n))$ time-computable $\vec{\beta}$-martingale that succeeds on $X$.

Notation. Given a bias sequence $\vec{\beta}=\left(\beta_{0}, \beta_{1}, \ldots\right), n \in \mathbb{N}$, and $S \in \mathbf{C}$, let

$$
\begin{aligned}
H_{n}(\vec{\beta}) & =\frac{1}{n} \sum_{i=0}^{n-1} \mathcal{H}\left(\beta_{i}\right), \\
H^{-}(\vec{\beta}) & =\liminf _{n \rightarrow \infty} H_{n}(\vec{\beta}), \\
H^{+}(\vec{\beta}) & =\limsup _{n \rightarrow \infty} H_{n}(\vec{\beta}) .
\end{aligned}
$$

We call $H^{-}(\vec{\beta})$ and $H^{+}(\vec{\beta})$ the lower and upper average entropies, respectively, of $\vec{\beta}$. Also, let

$$
L_{n}(\vec{\beta})(S)=\log \frac{1}{\mu^{\vec{\beta}}(S[0 . . n-1])}=\sum_{i=0}^{n-1} \xi_{i}(S),
$$


where

$$
\xi_{i}(S)=(1-S[i]) \log \frac{1}{1-\beta_{i}}+S[i] \log \frac{1}{\beta_{i}}
$$

for $0 \leq i<n$.

Note that $L_{n}(\vec{\beta}), \xi_{0}, \ldots, \xi_{n-1}$ are random variables with

$$
\mathrm{E} L_{n}(\vec{\beta})=\sum_{i=0}^{n-1} \mathrm{E} \xi_{i}=\sum_{i=0}^{n-1} \mathcal{H}\left(\beta_{i}\right)=n H_{n}(\vec{\beta})
$$

The following large deviation theorem tells us that $L_{n}(\vec{\beta})$ is very unlikely to deviate significantly from this expected value.

Theorem 5.29. For each $\delta>0$ and $\epsilon>0$, there exists $\alpha \in(0,1)$ such that, for all bias sequences $\vec{\beta}=\left(\beta_{0}, \beta_{1}, \ldots\right)$ with each $\beta_{i} \in[\delta, 1-\delta]$ and all $n \in \mathbb{Z}^{+}$, if $L_{n}(\vec{\beta})$ and $H_{n}(\vec{\beta})$ are defined as above, then

$$
\mathrm{P}\left[\left|L_{n}(\vec{\beta})-n H_{n}(\vec{\beta})\right| \geq \epsilon n\right]<2 \alpha^{n},
$$

where the probability is computed according to $\mu^{\vec{\beta}}$.

The proof of Theorem 5.29 is given in [5].

Lemma 5.30. If $\delta>0$ and $\vec{\beta}$ is an exactly $n^{l}$-time computable bias sequence with each $\beta_{i} \in[\delta, 1-\delta]$, then every sequence $R \in \operatorname{RAND}^{\vec{\beta}}\left(n^{k+2 l+1}\right)$ satisfies

$$
L_{n}(\vec{\beta})(R)=n H_{n}(\vec{\beta})+o(n)
$$

as $n \rightarrow \infty$.

Proof. Assume the hypothesis. Let $\epsilon>0$. For each $n \in \mathbb{N}$, define the set

$$
Y_{n}=\left\{S \in \mathbf{C}|| L_{n}(\vec{\beta})(S)-n H_{n}(\vec{\beta}) \mid \geq \epsilon n\right\},
$$

and let

$$
X_{\epsilon}=\left\{S \in \mathbf{C} \mid\left(\exists^{\infty} n\right) S \in Y_{n}\right\} .
$$


It suffices to show that $\mu_{n^{k+2 l+1}}^{\vec{\beta}}\left(X_{\epsilon}\right)=0$.

For each $n \in \mathbb{N}$ and $w \in\{0,1\}^{*}$, let

$$
d_{n}(w)= \begin{cases}\mu^{\vec{\beta}}\left(Y_{n} \mid \mathbf{C}_{w}\right) & \text { if }|w| \leq n \\ d_{n}(w[0 . . n-1]) & \text { if }|w|>n\end{cases}
$$

It is easily verified that each $d_{n}$ is a $\vec{\beta}$-martingale. It is clear that $Y_{n} \subseteq S^{1}\left[d_{n}\right]$ for all $n \in \mathbb{N}$. Finally, by Theorem 5.29, the series $\sum_{n=0}^{\infty} d_{n}(\lambda)$ is p-convergent, so the computable first BorelCantelli Lemma [33] (extended to $\vec{\beta}$ as indicated in [8]) tells us that $X_{\epsilon}$ has p-measure 0. It can be verified that the resulting martingale is computable in $n^{k+2 l+1}$ time, so $\mu_{n^{k+2 l+1}}^{\vec{\beta}}\left(X_{\epsilon}\right)=$ 0 .

Lemma 5.31. Assume that $k, l \in \mathbb{Z}^{+}, \delta>0, \vec{\beta}$ is an exactly $n^{l}$-time-computable bias sequence with each $\beta_{i} \in \mathbb{Q} \cap[\delta, 1-\delta], s \in \mathbb{Q} \cap[0, \infty)$, and $d$ is an $n^{k}$-time-computable s-gale.

1. If $s<H^{-}(\vec{\beta})$, then $S^{\infty}[d] \bigcap \operatorname{RAND}^{\vec{\beta}}\left(n^{k+2 l+1}\right)=\emptyset$.

2. If $s<H^{+}(\vec{\beta})$, then $S_{\mathrm{str}}^{\infty}[d] \bigcap \operatorname{RAND}^{\vec{\beta}}\left(n^{k+2 l+1}\right)=\emptyset$.

Proof. Assume the hypothesis. Define $d^{\prime}:\{0,1\}^{*} \rightarrow[0, \infty)$ by

$$
d^{\prime}(w)=\frac{d(w)}{2^{s|w|} \mu^{\vec{\beta}}(w)}
$$

for all $w \in\{0,1\}^{*}$. Then $d^{\prime}$ is a $\vec{\beta}$-martingale, and $d^{\prime}$ is $\mathrm{O}\left(n^{k+2 l+1}\right)$-time-computable.

Let $R \in \operatorname{RAND}^{\vec{\beta}}\left(n^{k+2 l+1}\right)$. Then $d^{\prime}$ does not succeed on $R$, so there is a constant $c>0$ such that, for all $n \in \mathbb{N}$, if we write $z_{n}=R[0 . . n-1]$, then $d^{\prime}\left(z_{n}\right) \leq 2^{c}$, whence

$$
\log d\left(z_{n}\right) \leq c+s n+\log \mu^{\vec{\beta}}\left(z_{n}\right)
$$

It follows by Lemma 5.30 that

$$
\log d\left(z_{n}\right) \leq c+n\left[s-H_{n}(\vec{\beta})\right]+o(n)
$$


as $n \rightarrow \infty$. Hence, for any $\epsilon>0$, if we let

$$
I_{\epsilon}=\left\{n \in \mathbb{Z}^{+} \mid s<H_{n}(\vec{\beta})-\epsilon\right\}
$$

then $\log d\left(z_{n}\right)<c$ for all sufficiently large $n \in I_{\epsilon}$. We now verify the two parts of the lemma.

1. If $s<H^{-}(\vec{\beta})$, let $\epsilon=\frac{H^{-}(\vec{\beta})-s}{2}$. Then $I_{\epsilon}$ is cofinite, so $\log d\left(z_{n}\right)<c$ for all sufficiently large $n \in \mathbb{Z}^{+}$, so $R \notin S^{\infty}[d]$.

2. If $s<H^{+}(\vec{\beta})$, let $\epsilon=\frac{H^{+}(\vec{\beta})-s}{2}$. Then $I_{\epsilon}$ is infinite, so $\log d\left(z_{n}\right)<c$ for infinitely many $n \in \mathbb{Z}^{+}$, so $R \notin S_{\text {str }}^{\infty}[d]$.

Our proof of Theorem 5.27 also uses the martingale dilation technique, which was introduced by Ambos-Spies, Terwijn, and Zheng [4] and extended by Breutzmann and Lutz $[8]$.

Definition. The restriction of a string $w \in\{0,1\}^{*}$ to a language $A \subseteq\{0,1\}^{*}$ is the string $w \uparrow A$ defined by the following recursion.

1. $\lambda\lceil A=\lambda$.

2. For $w \in\{0,1\}^{*}$ and $b \in\{0,1\}$,

$$
(w b) \uparrow A= \begin{cases}(w \uparrow A) b & \text { if } s_{|w|} \in A, \\ w \uparrow A & \text { if } s_{|w|} \notin A .\end{cases}
$$

(That is, $w \uparrow A$ is the concatenation of the successive bits $w[i]$ for which $s_{i} \in A$.)

Definition. A function $f:\{0,1\}^{*} \longrightarrow\{0,1\}^{*}$ is strictly increasing if, for all $x, y \in\{0,1\}^{*}$,

$$
x<y \Longrightarrow f(x)<f(y)
$$

where $<$ is the standard ordering of $\{0,1\}^{*}$. 
Notation. If $f:\{0,1\}^{*} \longrightarrow\{0,1\}^{*}$, then for each $n \in \mathbb{N}$, let $n_{f}$ be the unique integer such that $f\left(s_{n}\right)=s_{n_{f}}$.

Definition. If $f:\{0,1\}^{*} \longrightarrow\{0,1\}^{*}$ is strictly increasing and $\vec{\beta}$ is a bias sequence, then the $f$-dilation of $\vec{\beta}$ is the bias sequence $\vec{\beta}^{f}$ given by $\beta_{n}^{f}=\beta_{n_{f}}$ for all $n \in \mathbb{N}$.

Observation 5.32. If $f:\{0,1\}^{*} \longrightarrow\{0,1\}^{*}$ is strictly increasing and $A \subseteq\{0,1\}^{*}$, then for all $n \in \mathbb{N}$,

$$
\chi_{f^{-1}(A)}[0 . . n-1]=\chi_{A}\left[0 . . n_{f}-1\right]\lceil\operatorname{range}(f) .
$$

Definition. If $f:\{0,1\}^{*} \longrightarrow\{0,1\}^{*}$ is strictly increasing and $d$ is a martingale, then the $f$-dilation of $d$ is the function $f^{\wedge} d:\{0,1\}^{*} \longrightarrow[0, \infty)$,

$$
f^{\wedge} d(w)=d(w\lceil\operatorname{range}(f)) .
$$

Intuitively, the $f$-dilation of $d$ is a strategy for betting on a language $A$, assuming that $d$ itself is a good betting strategy for betting on the language $f^{-1}(A)$. Given an opportunity to bet on the membership of a string $y=f(x)$ in A, $f^{\wedge} d$ bets exactly as $d$ would bet on the membership or nonmembership of $x$ in $f^{-1}(A)$.

The following result is a special case of Theorem 6.3 in [8].

Theorem 5.33. (Martingale Dilation Theorem - Breutzmann and Lutz [8]) Assume that $\vec{\beta}$ is a bias sequence with each $\beta_{i} \in(0,1), f:\{0,1\}^{*} \longrightarrow\{0,1\}^{*}$ is strictly increasing, and $d$ is a $\vec{\beta}^{f}$-martingale. Then $f^{\wedge} d$ is a $\vec{\beta}$-martingale and, for every language $A \subseteq\{0,1\}^{*}$, if $d$ succeeds on $f^{-1}(A)$, then $f^{\wedge} d$ succeeds on $A$.

Notation. For each $k \in \mathbb{Z}^{+}$, define $g_{k}:\{0,1\}^{*} \longrightarrow\{0,1\}^{*}$ by $g_{k}(x)=0^{|x|^{k}} 1 x$. Note that each $g_{k}$ is strictly increasing and computable in polynomial time.

Lemma 5.34. Assume that $\vec{\beta}$ is a bias sequence with each $\beta_{i} \in(0,1)$, and $R \in \operatorname{RAND}^{\vec{\beta}}\left(n^{2}\right)$. Then, for each $k \geq 2, g_{k}^{-1}(R) \in \operatorname{RAND}^{\vec{\alpha}}\left(n^{k}\right)$, where $\vec{\alpha}=\vec{\beta}^{g_{k}}$.

Proof. Let $\vec{\beta}, k$, and $\vec{\alpha}$ be as given, and assume that $g_{k}^{-1}(R) \notin \operatorname{RAND}^{\vec{\alpha}}\left(n^{k}\right)$. Then there is an $n^{k}$-time-computable $\vec{\alpha}$-martingale $d$ that succeeds on $g_{k}^{-1}(R)$. It follows by Theorem 5.33 
that $g_{k} \hat{d}$ is a $\vec{\beta}$-martingale that succeeds on $R$. The time required to compute $g_{k} \hat{d}(w)$ is $\mathrm{O}\left(|w|^{2}+\left|w^{\prime}\right|^{k}\right)$ steps, where $w^{\prime}=w\left\lceil\operatorname{range}\left(g_{k}\right)\right.$. (This allows $\mathrm{O}\left(|w|^{2}\right)$ steps to compute $w^{\prime}$ and then $\mathrm{O}\left(|w|^{k}\right.$ steps to compute $d\left(w^{\prime}\right)$.) Now $\left|w^{\prime}\right|$ is bounded above by the number of strings $x$ such that $|x|^{k}+|x|+1 \leq\left|s_{|w|}\right|=\lfloor\log (1+|w|)\rfloor$, so $\left|w^{\prime}\right| \leq 2^{1+\log (1+|w|)^{\frac{1}{k}}}$. Therefore the time required to compute $g_{k} \hat{d}(w)$ is

$$
\mathrm{O}\left(|w|^{2}+2^{k} 2^{k(\log (1+|w|))^{\frac{1}{k}}}\right)=\mathrm{O}\left(|w|^{2}\right)
$$

steps. Thus $g_{k} \hat{d}(w)$ is an $n^{2}$-time computable $\vec{\beta}$-martingale, so $R \notin \operatorname{RAND}^{\vec{\beta}}\left(n^{2}\right)$.

Notation. From here through the proof of Theorem 5.27, we assume that $\alpha$ and $\beta$ are $\Delta_{2^{-}}^{0}$ computable real numbers with $0 \leq \alpha \leq \beta \leq 1 / 2$. It is well-known that a real number is $\Delta_{2}^{0}$-computable if and only if there is a computable sequence of rationals that converge to it. Slowing down this construction gives polynomial-time functions $\hat{\alpha}, \hat{\beta}: \mathbb{N} \rightarrow \mathbb{Q}$ such that $\lim _{n \rightarrow \infty} \hat{\alpha}(n)=\alpha$ and $\lim _{n \rightarrow \infty} \hat{\beta}(n)=\beta$. We also assume that $\frac{1}{n} \leq \hat{\alpha}(n) \leq \hat{\beta}(n)$ for all $n$. For each $n$, we let

$$
\kappa(n)= \begin{cases}\hat{\alpha}(n) & \text { if } n \text { is even } \\ \hat{\beta}(n) & \text { if } n \text { is odd }\end{cases}
$$

and define a special-purpose bias sequence $\vec{\gamma}$ by

$$
\gamma_{n}=\kappa\left(\log ^{*} n\right)
$$

Note that $\vec{\gamma}$ is $\mathrm{O}(n)$-time-computable, $\frac{1}{\log ^{*} n} \leq \gamma_{n}$ for all $n, H^{-}(\vec{\gamma})=\mathcal{H}(\alpha)$, and $H^{+}(\vec{\gamma})=$ $\mathcal{H}(\beta)$.

We now use the unpredictability characterizations from Theorem 3.33 to establish upper bounds on the dimensions and strong dimensions of lower spans of sequences random relative to $\vec{\gamma}$. 
Lemma 5.35. For each $R \in \operatorname{RAND}^{\vec{\gamma}}\left(n^{5}\right)$,

$$
\operatorname{dim}_{\mathrm{p}}\left(\mathrm{P}_{\mathrm{m}}(R)\right) \leq \mathcal{H}(\alpha)
$$

and

$$
\operatorname{Dim}_{\mathrm{p}}\left(\mathrm{P}_{\mathrm{m}}(R)\right) \leq \mathcal{H}(\beta)
$$

Proof. For now, fix a polynomial-time function $f:\{0,1\}^{*} \rightarrow\{0,1\}^{*}$. The collision set of $f$ is

$$
C_{f}=\left\{j \mid(\exists i<j) f\left(s_{i}\right)=f\left(s_{j}\right)\right\}
$$

For each $n \in \mathbb{N}$, let

$$
\# C_{f}(n)=\left|C_{f} \cap\{0, \ldots, n-1\}\right|
$$

We use $f$ to define the predictors

$$
\pi_{0}^{f}(w, b)= \begin{cases}\frac{1}{2} & \text { if }|w| \notin C_{f} \\ w[i]^{b}(1-w[i])^{1-b} & \text { if }|w| \in C_{f} \text { and } i=\min \left\{j \mid f\left(s_{j}\right)=f\left(s_{|w|}\right)\right\}\end{cases}
$$

and

$$
\pi_{1}^{f}(w, b)= \begin{cases}\left(\gamma_{|w|}^{f}\right)^{b}\left(1-\gamma_{|w|}^{f}\right)^{1-b} & \text { if }|w| \notin C_{f} \\ w[i]^{b}(1-w[i])^{1-b} & \text { if }|w| \in C_{f} \text { and } i=\min \left\{j \mid f\left(s_{j}\right)=f\left(s_{|w|}\right)\right\}\end{cases}
$$

for all $w \in\{0,1\}^{*}$ and $b \in\{0,1\}$.

For each $S \in \mathbf{C}$, we now define several objects to facilitate the proof. First, we let

$$
A^{f}(S)=f^{-1}(S)
$$


that is, $A^{f}(S)$ is the language $\leq{ }_{\mathrm{m}}^{\mathrm{p}}$-reduced to $S$ by $f$. Observe that for all $w \sqsubseteq A^{f}(S)$,

$$
\mathcal{L}^{\log }\left(\pi_{0}^{f}, w\right)=|w|-\# C_{f}(|w|)
$$

Recall the sequence of towers defined by $t_{j}$ by $t_{0}=1$ and $t_{j+1}=2^{t_{j}}$. For any $j \in \mathbb{N}$ and $t_{j}<n \leq t_{j+1}$, define the entropy quantity

$$
H_{n}^{f}=\sum_{\substack{i<n \\ i \notin C_{f} \text { and } i_{f}>t_{j-1}}} \mathcal{H}\left(\gamma_{n}^{f}\right)
$$

and the random variable

$$
L_{n}^{f}(S)=\sum_{\substack{i<n \\ i \notin C_{f} \text { and } i_{f}>t_{j-1}}} \log \frac{1}{\pi_{1}^{f}\left(A^{f}(S)[0 . . i-1], A^{f}(S)[i]\right)} .
$$

(Recall that $i_{f}$ is the unique number such that $f\left(s_{i}\right)=s_{i_{f}}$.) We have

$$
\begin{aligned}
\mathcal{L}^{\log }\left(\pi_{1}^{f}, A^{f}(S)[0 . . n-1]\right) & =\sum_{i<n} \log \frac{1}{\pi_{1}^{f}\left(A^{f}(S)[0 . . i-1], A^{f}(S)[i]\right)} \\
& =\sum_{\substack{i<n \\
i \notin C_{f}}} \log \frac{1}{\pi_{1}^{f}\left(A^{f}(S)[0 . . i-1], A^{f}(S)[i]\right)} \\
& =L_{n}^{f}(S)+\sum_{\substack{i \notin n \\
i \notin C_{f} \text { and } i_{f} \leq t_{j-1}}} \log \frac{1}{\pi_{1}^{f}\left(A^{f}(S)[0 . . i-1], A^{f}(S)[i]\right)} \\
& \leq L_{n}^{f}(S)+\sum_{i \notin C_{f} \text { and } i_{f} \leq t_{j-1}} \log \log ^{*} i_{f} \\
& \leq L_{n}^{f}(S)+\left(t_{j-1}+1\right) \log (j-1) \\
& \leq L_{n}^{f}(S)+(1+\log n) \log ^{*} n,
\end{aligned}
$$

for all $n$. (Here we used the fact that $\gamma_{i} \geq \frac{1}{\log ^{*} i}$ for all $i$.) Finally, for any $\epsilon>0$ and $\theta \in(0,1)$, define the set

$$
J_{\theta, \epsilon}^{f}(S)=\left\{n \mid \# C_{f}(n)<(1-\theta) n \text { and } L_{n}^{f}(S) \geq H_{n}^{f}+\epsilon n\right\}
$$


of natural numbers.

Claim. For any rational $\theta \in(0,1)$ and $\epsilon>0$,

$$
\mu_{n^{5}}^{\vec{\gamma}}\left(\left\{S \mid J_{\theta, \epsilon}^{f}(S) \text { is finite }\right\}\right)=1
$$

Proof of Claim. The argument is similar to the proof of Lemma 5.30. For each $n \in \mathbb{N}$, define the set

$$
Y_{n}= \begin{cases}\emptyset & \text { if } \# C_{f}(n) \geq(1-\theta) n \\ \left\{S \mid L_{n}^{f}(S) \geq H_{n}^{f}+\epsilon n\right\} & \text { otherwise, }\end{cases}
$$

and let

$$
X_{\epsilon}=\left\{S \in \mathbf{C} \mid\left(\exists^{\infty} n\right) S \in Y_{n}\right\}
$$

To prove the claim, we will show that $\mu_{n^{5}}\left(X_{\epsilon}\right)=0$.

For each $n \in \mathbb{N}$ and $w \in\{0,1\}^{*}$, let

$$
d_{n}(w)= \begin{cases}\mu^{\vec{\gamma}}\left(Y_{n} \mid \mathbf{C}_{w}\right) & \text { if }|w| \leq n \\ d_{n}(w[0 . . n-1]) & \text { if }|w|>n\end{cases}
$$

It is clear that each $d_{n}$ is a $\vec{\gamma}$-martingale and that $Y_{n} \subseteq S^{1}\left[d_{n}\right]$ for all $n \in \mathbb{N}$.

Let $S \in \mathbf{C}$. For each $n, j \in \mathbb{N}$, let

$$
I_{j}^{n}=\left\{i_{f} \mid i<n, i \notin C_{f}, \text { and } \log ^{*} i_{f}=j\right\}
$$

Also, define $S^{+}=\{i \mid S[i]=1\}$ and $S^{-}=\{i \mid S[i]=0\}$. Then, if $n$ is large enough to ensure that $\log ^{*} i_{f} \leq 1+\log ^{*} n$ for all $i<n$, we have

$$
L_{n}^{f}(S)=\sum_{k=\left(\log ^{*} n\right)-1}^{\left(\log ^{*} n\right)+1}\left|I_{k}^{n} \cap S^{+}\right| \log \frac{1}{\kappa(k)}+\left|I_{k}^{n} \cap S^{-}\right| \log \frac{1}{1-\kappa(k)}
$$

For any $n$ and $k$, write $i(n, k)=\left|I_{k}^{n}\right|$. Let $T_{n}$ be the set of all tuples $\left(l_{-1}, l_{0}, l_{1}\right)$ satisfying 
$0 \leq l_{r} \leq i(n, j+r)$ for $-1 \leq r \leq 1$ and

$$
\sum_{r=-1}^{1} l_{r} \log \frac{1}{\kappa(j+r)}+\left(i(n, j+r)-l_{r}\right) \log \frac{1}{1-\kappa(j+r)} \geq H_{n}^{f}+\epsilon n,
$$

where $j=\log ^{*} n$. Then we have

$$
\mu^{\vec{\gamma}}\left(Y_{n}\right)=\sum_{\left(l_{-1}, l_{0}, l_{1}\right) \in T_{n}} \prod_{r=-1}^{1}\left(\begin{array}{c}
i(n, j+r) \\
l_{r}
\end{array}\right) \kappa(j+r)^{l_{r}}(1-\kappa(j+r))^{i(n, j+r)-l_{r}}
$$

We can write a similar formula for $\mu^{\vec{\gamma}}\left(Y_{n} \mid \mathbf{C}_{w}\right)$ when $w \neq \lambda$. From this it follows that the mapping $(n, w) \mapsto d_{n}(w)$ is exactly computable in $O\left(n^{3}\right)$ time.

By Theorem 5.29, there exists $\delta \in(0,1)$ such that for all $n \in \mathbb{N}$ with $Y_{n} \neq \emptyset$, we have

$$
\mu^{\vec{\gamma}}\left(Y_{n}\right)<2 \delta^{n-\# C_{f}(n)}<2 \delta^{\theta n}
$$

It follows that the series $\sum_{n=0}^{\infty} d_{n}(\lambda)$ is p-convergent, so the polynomial-time first Borel-Cantelli Lemma [33] (extended to $\vec{\gamma}$ as indicated in [8]) tells us that $\mu_{n^{5}}^{\vec{\gamma}}\left(X_{\epsilon}\right)=0 . \quad \square$ Claim.

Let $R \in \operatorname{RAND}^{\vec{\gamma}}\left(n^{5}\right)$. Let $\epsilon>0$ and $\theta<\mathcal{H}(\alpha)$ be rational. Then by the above claim, $J_{\theta, \epsilon}^{f}(R)$ is finite. That is, for all but finitely many $n$,

$$
\# C_{f}(n) \geq(1-\theta) n \text { or } L_{n}^{f}(R)<H_{n}^{f}+\epsilon n
$$

Writing $w_{n}=A^{f}(R)[0 . . n-1],(5.5)$ combined with (5.3) and (5.4) implies that

$$
\mathcal{L}^{\log }\left(\pi_{0}^{f}, w_{n}\right) \leq \theta n<\mathcal{H}(\alpha) n
$$

or

$$
\mathcal{L}^{\log }\left(\pi_{1}^{f}, w_{n}\right)<H_{n}^{f}+\epsilon n+(1+\log n) \log ^{*} n
$$

As

$$
\limsup _{n \rightarrow \infty} \frac{H_{n}^{f}}{n} \leq \mathcal{H}(\beta)
$$


it follows that

$$
\limsup _{n \rightarrow \infty} \frac{\min \left\{\mathcal{L}^{\log }\left(\pi_{0}^{f}, w_{n}\right), \mathcal{L}^{\log }\left(\pi_{1}^{f}, w_{n}\right)\right\}}{n} \leq \mathcal{H}(\beta)+\epsilon
$$

If (5.6) holds for infinitely many $n$, then

$$
\mathcal{L}^{\log }\left(\pi_{0}^{f}, A^{f}(R)\right) \leq \mathcal{H}(\alpha)
$$

Otherwise, (5.7) holds for almost all $n$. Assuming

$$
\liminf _{n \rightarrow \infty} \frac{H_{n}^{f}}{n} \leq \mathcal{H}(\alpha)
$$

in this case we have

$$
\mathcal{L}^{\log }\left(\pi_{1}^{f}, A^{f}(R)\right) \leq \mathcal{H}(\alpha)+\epsilon
$$

We now verify (5.10). For each $n$, let $m(n)=t_{n}^{2}$. Then for sufficiently large $n$, we have $i_{f}<t_{n+1}$ for all $i<m(n)$. Using the sets $I_{n}^{k}$ from the proof of the claim, we then have

$$
\begin{aligned}
H_{m(n)}^{f} & =\left|I_{n}^{m(n)}\right| \mathcal{H}(\kappa(n))+\left|I_{n+1}^{m(n)}\right| \mathcal{H}(\kappa(n+1)) \\
& \leq\left(t_{n}+1\right) \mathcal{H}(\kappa(n))+m(n) \mathcal{H}(\kappa(n+1)) .
\end{aligned}
$$

As $t_{n}=o(m(n))$ and $\kappa(2 n) \rightarrow \alpha$ as $n \rightarrow \infty$, we have

$$
\liminf _{n \rightarrow \infty} \frac{H_{n}^{f}}{n} \leq \liminf _{n \rightarrow \infty} \frac{H_{m(2 n+1)}^{f}}{m(2 n+1)} \leq \mathcal{H}(\alpha) .
$$

For each polynomial-time reduction $f$, we have defined and analyzed two predictors $\pi_{0}^{f}$ and $\pi_{1}^{f}$. We now show how to combine all these predictors into a single predictor that will establish the lemma.

Let $\left\{f_{j} \mid j \in \mathbb{N}\right\}$ be a uniform enumeration of all polynomial-time functions $f_{j}:\{0,1\}^{*} \rightarrow$ $\{0,1\}^{*}$ such that $f_{j}(x)$ is computable in $O\left(2^{|x|}+j\right)$ steps. For any predictor $\rho$, define a 
probability measure $\mu[\rho]$ by

$$
\mu[\rho](w)=\prod_{i=0}^{|w|-1} \rho(w[0 . . i-1], w[i])
$$

for all $w \in\{0,1\}^{*}$. For each $m \in \mathbb{N}$ and $w \in\{0,1\}^{m}$, let

$$
\mu_{m}(w)=2^{-(2 m+1)}+\sum_{j=0}^{m-1} 2^{-(2 j+3)}\left(\mu\left[\pi_{0}^{f_{j}}\right](w)+\frac{1}{2} \mu\left[\pi_{1}^{f_{j}}\right](w)\right)
$$

Then

$$
\begin{aligned}
\mu_{m+1}(w 0)+\mu_{m+1}(w 1)= & 2^{-(2 m+3)}+\sum_{j=0}^{m} 2^{-(2 j+3)}\left(\mu\left[\pi_{0}^{f_{j}}\right](w 0)+\frac{1}{2} \mu\left[\pi_{1}^{f_{j}}\right](w 0)\right) \\
& +2^{-(2 m+3)}+\sum_{j=0}^{m} 2^{-(2 j+3)}\left(\mu\left[\pi_{0}^{f_{j}}\right](w 1)+\frac{1}{2} \mu\left[\pi_{1}^{f_{j}}\right](w 1)\right) \\
= & 2^{-(2 m+2)}+\sum_{j=0}^{m} 2^{-(2 j+3)}\left(\mu\left[\pi_{0}^{f_{j}}\right](w)+\frac{1}{2} \mu\left[\pi_{1}^{f_{j}}\right](w)\right) \\
= & 2^{-(2 m+3)}\left(2+\mu\left[\pi_{0}^{f_{m}}\right](w)+\frac{1}{2} \mu\left[\pi_{1}^{f_{m}}\right](w)\right)+\mu_{m}(w)-2^{-(2 m+1)} \\
\leq & \mu_{m}(w)+2^{-(2 m+3)}\left(3+\frac{1}{2}\right)-2^{-(2 m+1)} \\
< & \mu_{m}(w) .
\end{aligned}
$$

Now define a predictor $\pi$ by

$$
\begin{aligned}
& \pi(w, 1)=\frac{\mu_{|w|+1}(w 1)}{\mu_{|w|}(w)} \\
& \pi(w, 0)=1-\pi(w, 1) .
\end{aligned}
$$

Then for all $w \in\{0,1\}^{*}$ and $b \in\{0,1\}$,

$$
\pi(w, b) \geq \frac{\mu_{|w|+1}(w b)}{\mu_{|w|}(w)} .
$$


For all $w \in\{0,1\}^{*}, k \in\{0,1\}$, and $j<|w|$, we have

$$
\begin{aligned}
\mathcal{L}^{\log }(\pi, w) & =\sum_{i=0}^{|w|-1} \log \frac{1}{\pi(w[0 . . i-1], w[i])} \\
& \leq \sum_{i=0}^{|w|-1} \log \frac{\mu_{i}(w[0 . . i-1])}{\mu_{i+1}(w[0 . . i])} \\
& =\log \frac{\mu_{0}(\lambda)}{\mu_{|w|}(w)} \\
& \leq \log \frac{2^{2 j+3+i}}{\mu\left[\pi_{k}^{f_{j}}\right](w)} \\
& =2 j+3+i+\mathcal{L}^{\log }\left(\pi_{k}^{f_{j}}, w\right) .
\end{aligned}
$$

For any $j \in \mathbb{N}$, it follows that

$$
\mathcal{L}_{\mathrm{str}}^{\log }\left(\pi, A^{f_{j}}(R)\right) \leq \mathcal{H}(\beta)+\epsilon
$$

by using $f=f_{j}$ in (5.8). Also, since either (5.9) or (5.11) holds for $f=f_{j}$, we have

$$
\mathcal{L}^{\log }\left(\pi, A^{f_{j}}(R)\right) \leq \mathcal{H}(\alpha)+\epsilon
$$

As $\pi$ is (exactly) polynomial-time computable, this establishes that

$$
\mathrm{P}_{\mathrm{m}}(R)=\left\{A^{f_{j}}(R) \mid j \in \mathbb{N}\right\}
$$

has p-dimension at most $\mathcal{H}(\alpha)+\epsilon$ and strong p-dimension at most $\mathcal{H}(\beta)+\epsilon$ by Theorem 3.33. As $\epsilon>0$ was arbitrary, the lemma follows.

We now have the machinery we need to prove the main result of this section.

Proof of Theorem 5.27. Let $x$ and $y$ be $\Delta_{2}^{0}$-computable real numbers with $0 \leq x \leq y \leq 1$. Then there exist $\Delta_{2}^{0}$-computable real numbers $\alpha$ and $\beta$ with $0 \leq \alpha \leq \beta \leq \frac{1}{2}, \mathcal{H}(\alpha)=x$, and $\mathcal{H}(\beta)=y$. Let $\vec{\gamma}$ be the bias sequence defined from $\alpha$ and $\beta$ above (just prior to Lemma 5.35). It is well-known $[33,4]$ that almost every language in $\mathrm{E}$ is $n^{5}-\vec{\gamma}$-random. In particular, there 
exists a language $A \in \operatorname{RAND}^{\vec{\beta}}\left(n^{5}\right) \cap$ E. By Theorems 5.2 and 5.28 , it suffices to prove that

$$
\operatorname{dim}_{\mathrm{p}}\left(\mathrm{P}_{\mathrm{m}}(A)\right)=\operatorname{dim}\left(\mathrm{P}_{\mathrm{m}}(A) \mid \mathrm{E}\right)=\mathcal{H}(\alpha)
$$

and

$$
\operatorname{Dim}_{\mathrm{p}}\left(\mathrm{P}_{\mathrm{m}}(A)\right)=\operatorname{Dim}\left(\mathrm{P}_{\mathrm{m}}(A) \mid \mathrm{E}\right)=\mathcal{H}(\beta)
$$

By Lemma 5.35, then, it suffices to prove that

$$
\operatorname{dim}\left(\mathrm{P}_{\mathrm{m}}(A) \mid \mathrm{E}\right) \geq \mathcal{H}(\alpha)
$$

and

$$
\operatorname{Dim}\left(\mathrm{P}_{\mathrm{m}}(A) \mid \mathrm{E}\right) \geq \mathcal{H}(\beta)
$$

Note that (5.12) is trivial if $\alpha=0$, and (5.13) is trivial if $\beta=0$. If $\alpha>0$, let $s \in[0, \mathcal{H}(\alpha)) \cap \mathbb{Q}$, and let $d^{-}$be an $n^{k}$-time computable $s$-gale. Similarly, if $\beta>0$, let $t \in[0, \mathcal{H}(\beta)) \cap \mathbb{Q}$, and let $d^{+}$be an $n^{k}$-time computable $t$-gale. It suffices to show that

$$
\alpha>0 \Rightarrow \mathrm{P}_{\mathrm{m}}(A) \cap \mathrm{E} \nsubseteq S^{\infty}\left[d^{-}\right]
$$

and

$$
\beta>0 \Rightarrow \mathrm{P}_{\mathrm{m}}(A) \cap \mathrm{E} \not \subseteq S_{\mathrm{str}}^{\infty}\left[d^{+}\right]
$$

Let $B=g_{k+3}^{-1}(A)$. It is clear that $B \in \mathrm{P}_{\mathrm{m}}(A) \cap \mathrm{E}$. Also, by Lemma 5.34, $B \in \operatorname{RAND}^{\vec{\gamma}^{\prime}}\left(n^{k}\right)$, where $\vec{\gamma}^{\prime}=\vec{\gamma}^{g_{k+3}}$. Since

$$
\begin{aligned}
& s<\mathcal{H}(\alpha)=H^{-}(\vec{\gamma})=H^{-}\left(\vec{\gamma}^{\prime}\right), \\
& t<\mathcal{H}(\beta)=H^{+}(\vec{\gamma})=H^{+}\left(\vec{\gamma}^{\prime}\right),
\end{aligned}
$$

and $\vec{\gamma}^{\prime}$ is $\mathrm{O}(n)$-time-computable, Lemma 5.31 tells us that $\alpha>0 \Rightarrow B \notin S^{\infty}\left[d^{-}\right]$and $\beta>0 \Rightarrow$ $B \notin S_{\text {str }}^{\infty}\left[d^{+}\right]$. Thus (5.14) and (5.15) hold. 
In light of Theorem 5.27, the following question concerning the relativized feasible dimension of NP is natural.

Open Question 5.36. For which pairs of real numbers $\alpha, \beta \in[0,1]$ does there exist an oracle A such that $\operatorname{dim}_{\mathrm{p}^{A}}\left(\mathrm{NP}^{A}\right)=\alpha$ and $\operatorname{Dim}_{\mathrm{p}^{A}}\left(\mathrm{NP}^{A}\right)=\beta$ ?

\subsection{Reductions to Nondense Languages}

The hypothesis that NP does not have p-measure 0 , written $\mu_{\mathrm{p}}(\mathrm{NP}) \neq 0$, has been used extensively in computational complexity theory and has been shown to have many plausible consequences that are not known to follow from the weaker $\mathrm{P} \neq \mathrm{NP}$ hypothesis. If $\mu_{\mathrm{p}}(\mathrm{NP}) \neq 0$, then $\operatorname{dim}_{\mathrm{p}}(\mathrm{NP})=1$, but if $\mu_{\mathrm{p}}(\mathrm{NP})=0, \operatorname{dim}_{\mathrm{p}}(\mathrm{NP})$ could be any number between 0 and 1 . This means that the hypothesis that NP has positive p-dimension, written $\operatorname{dim}_{\mathrm{p}}(\mathrm{NP})>0$, is potentially weaker than $\mu_{\mathrm{p}}(\mathrm{NP}) \neq 0$.

This positive dimension hypothesis on NP was used in [19] to extend the inapproximability result of Håstad [16] for MAx3SAT. The main theorem of [19] asserts that if $\operatorname{dim}_{\mathrm{p}}(\mathrm{NP})>0$, then MAX3SAT is exponentially hard to approximate in that any approximation algorithm must either use exponential time or fail to approximate well on a set of exponential density.

Theorem 5.37. (Hitchcock [19]) If $\operatorname{dim}_{\mathrm{p}}(\mathrm{NP})>0$, then any approximation algorithm $\mathcal{A}$ for the MAX3SAT optimization problem must satisfy at least one of the following.

1. There is some $\delta>0$ such that $\mathcal{A}$ uses at least $2^{n^{\delta}}$ time.

2. For all $\epsilon>0, \mathcal{A}$ has performance ratio less than $\frac{7}{8}+\epsilon$ on an exponentially dense set of satisfiable instances.

Here we say that a language $B \subseteq\{0,1\}^{*}$ is (exponentially) dense if there is a $\delta>0$ such that for all sufficiently large $n,\left|B_{\leq n}\right| \geq 2^{n^{\delta}}$. Let DENSE be the class of all dense languages. The following theorem asserts that the classes of languages reducible to nondense languages under reductions with a restricted number of queries has p-measure 0.

Theorem 5.38. Let $\alpha<1$. 
1. (Lutz and Mayordomo [37]) $\mu_{\mathrm{p}}\left(\mathrm{P}_{n^{\alpha}-\mathrm{tt}}\left(\mathrm{DENSE}^{c}\right)\right)=0$.

2. (Lutz and Zhao [39]) $\mu_{\mathrm{p}}\left(\mathrm{P}_{n^{\alpha / 2}-\mathrm{T}}\left(\mathrm{DENSE}^{c}\right)\right)=0$.

It seems difficult to extend Theorem 5.38 from p-measure to p-dimension. However, the following result is true. This was a key ingredient in the proof of Theorem 5.37.

Theorem 5.39. (Hitchcock $[19]) \operatorname{dim}_{\mathrm{p}}\left(\mathrm{P}_{\mathrm{m}}\left(\mathrm{DENSE}^{c}\right)\right)=0$.

In this section we use the unpredictability characterization of dimension to extend Theorem 5.39 in two ways. Theorem 5.43 strengthens it from many-one reductions to conjunctive reductions. Before that, in Theorem 5.42, we give a related result for strong dimension. Both results make use of Theorem 3.33. The following two propositions about combining predictors will be useful in the proofs.

Proposition 5.40. Let $\left\{\pi_{l} \mid l \in \mathbb{N}\right\}$ be a family of predictors uniformly computable in $O\left(n^{k}\right)$ time. Then there is a predictor $\pi$ exactly computable in $O\left(n^{k+2}\right)$ time such that for all $l \in \mathbb{N}$, for all $w \in\{0,1\}^{>l}, \mathcal{L}^{\log }(\pi, w) \leq \mathcal{L}^{\log }\left(\pi_{l}, w\right)+c(l+1)$, where $c$ is a constant independent of $l$ and $w$.

Proof. Let $f$ be computable in $O\left((l+r+|w|)^{k}\right)$ time such that

$$
\left|f(l, w, b, r)-\pi_{l}(w, b)\right| \leq 2^{-r}
$$

for all $l, r \in \mathbb{N}, w \in\{0,1\}^{*}$, and $b \in\{0,1\}$. We can assume that

$$
f(l, w, b, r)=k 2^{-r}
$$

for some $1 \leq k \leq 2^{r}-1$ and that

$$
f(l, w, 0, r)+f(l, w, 1, r)=1 .
$$


For any $l \in \mathbb{N}, w \in\{0,1\}^{*}$, and $b \in\{0,1\}$, let

$$
\hat{\pi}_{n}(w, b)= \begin{cases}f(l, w, b,|w|)-2^{-|w|} & \text { if } f(l, w, b,|w|) \in\left(\frac{1}{2}, 1\right) \\ f(l, w, b,|w|)+2^{-|w|} & \text { if } f(l, w, b,|w|) \in\left(0, \frac{1}{2}\right) \\ f(l, w, b,|w|) & \text { if } f(l, w, b,|w|) \in\left\{0, \frac{1}{2}, 1\right\}\end{cases}
$$

Using the inequality

$$
\log \frac{1}{\hat{x}} \leq \log \frac{1}{x}+2^{-r+2}
$$

for $x \leq \hat{x}+2^{-r}$ when $\hat{x} \geq \frac{1}{2}$, notice that

$$
\mathcal{L}^{\log }\left(\hat{\pi}_{l}, w\right) \leq \mathcal{L}^{\log }\left(\pi_{l}, w\right)+\sum_{i=0}^{|w|-1} 2^{-i+2} \leq \mathcal{L}^{\log }\left(\pi_{l}, w\right)+8
$$

For any $l \in \mathbb{N}$ and $w \in\{0,1\}^{*}$, let

$$
\hat{\mu}_{l}(w)=\prod_{i=0}^{|w|-1} \hat{\pi}_{l}(w[0 . . i-1], w[i])
$$

For any $m \in \mathbb{N}$ and $w \in\{0,1\}^{m}$, define

$$
\rho_{m}(w)=2^{-(2 m+1)}+\sum_{j=0}^{m-1} 2^{-(2 j+3)} \hat{\mu}_{j}(w)
$$

Then we have

$$
\begin{aligned}
\rho_{m+1}(w 0)+\rho_{m+1}(w 1) & =2^{-(2 m+3)}+\sum_{j=0}^{m} 2^{-(2 j+3)} \hat{\mu}_{j}(w 0)+2^{-(2 m+3)}+\sum_{j=0}^{m} 2^{-(2 j+3)} \hat{\mu}_{j}(w 1) \\
& =2^{-(2 m+2)}+\sum_{j=0}^{m} 2^{-(2 j+3)} \hat{\mu}_{j}(w) \\
& =2^{-(2 m+3)}\left(2+\hat{\mu}_{m}(w)\right)+\rho_{m}(w)-2^{-(2 m+1)} \\
& \leq \rho_{m}(w)+2^{-(2 m+3)}(3-4) \\
& <\rho_{m}(w)
\end{aligned}
$$


for all $w \in\{0,1\}^{m}$. The predictor $\pi$ is defined by

$$
\begin{aligned}
& \pi(w, 1)=\frac{\rho_{|w|+1}(w b)}{\rho_{|w|}(w)} \\
& \pi(w, 0)=1-\pi(w, 1)
\end{aligned}
$$

for all $w \in\{0,1\}^{*}$. For any $l \in \mathbb{N}$, if $|w|>l$, we have

$$
\begin{aligned}
\mathcal{L}^{\log }(\pi, w) & =\sum_{i=0}^{|w|-1} \log \frac{1}{\pi(w[0 . . i-1], w[i])} \\
& \leq \sum_{i=0}^{|w|-1} \log \frac{\rho_{i}(w[0 . . i-1])}{\rho_{i+1}(w[0 . . i])} \\
& =\log \frac{\rho_{0}(\lambda)}{\rho_{|w|}(w)} \\
& \leq \log \frac{2^{2 l+3}}{\hat{\mu}_{l}(w)} \\
& =\mathcal{L}^{\log \left(\hat{\pi}_{l}, w\right)+2 l+3} \\
& \leq \mathcal{L}^{\log }\left(\pi_{n}, w\right)+2 l+11 .
\end{aligned}
$$

As $\pi(w, b)$ is exactly computable in time $O\left(|w|^{k+2}\right)$, the proposition holds.

The following proposition allows us to only be concerned with individual languages when investigating the dimension of a class. This proposition is similar to one given by Ambos-Spies, Merkle, Stephan, and Reimann [2].

Proposition 5.41. Let $\mathcal{C}$ be a class of languages and let $r \in \mathbb{N}$. If for each $A \in \mathcal{C}$ there is some predictor $\pi_{A}$ computable in $O\left(n^{r}\right)$ time such that $\mathcal{L}^{\log }\left(\pi_{A}, A\right) \leq s$, then $\operatorname{dim}_{\mathrm{p}}(\mathcal{C}) \leq s$. Analogously, if for each $A \in \mathcal{C}$ there is a predictor $\pi_{A}$ computable in $O\left(n^{r}\right)$ time satisfying $\mathcal{L}_{\text {str }}^{\log }\left(\pi_{A}, A\right) \leq s$, then $\operatorname{Dim}_{\mathrm{p}}(\mathcal{C}) \leq s$

Proof. This follows from Proposition 5.40 because the family of predictors computable in $O\left(n^{r}\right)$ time is uniformly computable in $O\left(n^{r+1}\right)$ time.

Now we come to the first main result of this section. Here we say that $B$ is i.o.-dense if 


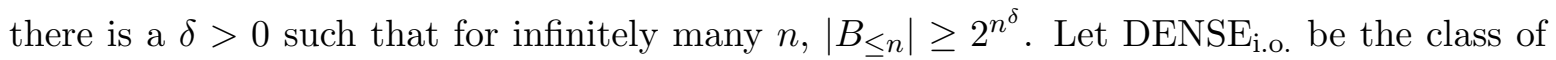
all i.o. dense languages.

Theorem 5.42. $\operatorname{Dim}_{\mathrm{p}}\left(\mathrm{P}_{\mathrm{ctt}}\left(\mathrm{DENSE}_{\mathrm{i} . \mathrm{o} .}^{c}\right)\right)=0$.

Proof. Assume that $A \leq_{\mathrm{ctt}}^{\mathrm{p}} D \in \mathrm{DENSE}_{\mathrm{i} . \mathrm{o}}^{c}$ via a reduction $f$ that is computable in $n^{r}$ time almost everywhere. For each $w \in\{0,1\}^{*}$, define the set of strings

$$
T_{w}=\bigcup_{i: w[i]=1} f\left(s_{i}\right)
$$

Then if we define

$$
T=\bigcup_{w \sqsubseteq A} T_{w}
$$

we have $T \subseteq D$ and $A \leq_{\mathrm{ctt}}^{\mathrm{p}} T$ via $f$. In fact, $T$ is the minimal set to which $A$ is conjunctively reduced via $f$. This useful idea comes from [9].

Let $k \geq 1$ and let $\epsilon=\frac{1}{k+1}$. Define a predictor $\pi$ for $A$ as follows.

- If $f\left(s_{|w|}\right) \subseteq T_{w}$, then $\pi(w, 1)=1$ and $\pi(w, 0)=0$.

- Otherwise, $\pi(w, 0)=1-\epsilon$ and $\pi(w, 1)=\epsilon$.

That is, when predicting the membership of a string $x$ in $A, \pi$ computes the portion of $T$ that has already been determined by the previous strings. If all the queries for $x$ are already known to be in $T$, then $\pi$ can be certain that $x$ is a member of $A$. Otherwise, $\pi$ predicts with high confidence that $x$ is not in $A$.

Observe that if $|w|=n, \pi(w, b)$ is computable in time $O\left(n(\log n)^{r}\right)=O\left(n^{2}\right)$. We will show that the strong log-loss rate of $\pi$ on $A$ is at most $\frac{2}{k}$. By Proposition 5.41, this will imply that

$$
\operatorname{Dim}_{\mathrm{p}}\left(\mathrm{P}_{\mathrm{ctt}}\left(\mathrm{DENSE}_{\text {i.o. }}^{c}\right)\right) \leq \frac{2}{k}
$$

As $k \geq 1$ is arbitrary, the theorem follows. 
To compute the loss of $\pi$ on $A$ we define an auxiliary sequence $J$ by

$$
J[n]=1 \Longleftrightarrow f\left(s_{n}\right) \subseteq T_{A[0 . . n-1]} .
$$

Intuitively, $J$ is the sequence of bits forecast by $\pi$, and our goal is to show that $J$ is close to $A$. We consider the following three cases for each $j \in \mathbb{N}$.

(1) $J[j]=1$ : This implies that $A[j]=1$ and $\log \frac{1}{\pi(A[0 . . j-1], A[j])}=\log 1=0$.

(2) $J[j]=0$ and $A[j]=1$ : Then $\log \frac{1}{\pi(A[0 . . j-1], A[j])}=\log \frac{1}{\epsilon}=\log (k+1)$.

(3) $J[j]=0$ and $A[j]=0$ : Then $\log \frac{1}{\pi(A[0 . . j-1], A[j])}=\log \frac{1}{1-\epsilon}=\log \frac{k+1}{k}$.

For each $i \in\{1,2,3\}$, let $\#_{i}(n)=\mid\{0 \leq j<n \mid$ Case $(i)$ holds for $j\} \mid$. Cases (1) and (3) are good for $\pi$. We will show that Case (2) is rare.

More specifically, we claim that

$$
\limsup _{n \rightarrow \infty} \frac{\#_{2}(n)}{n}=0
$$

To see this, let $\alpha$ be the value of this limit superior, suppose that $\alpha>0$, and let $0<\alpha^{\prime}<\alpha$. Whenever Case (2) holds for some $j$, we have $f\left(s_{j}\right) \nsubseteq T_{A[0 . . j-1]}$ and $A[j]=1$, so $\left|T_{A[0 . . j]}\right|>$ $\left|T_{A[0 . . j-1]}\right|$. This implies that $\left|T_{A[0 . . n]}\right| \geq \#_{2}(n)$ for all $n$. We then have infinitely many $n$ for which

$$
\left|T_{A[0 . . n]}\right| \geq \alpha^{\prime} n
$$

Suppose that (5.1) holds for some $m$ where $2^{n}-1<m \leq 2^{n+1}-1$. Then

$$
\left|T_{A\left[0.2^{n+1}-1\right]}\right| \geq\left|T_{A[0 . . m]}\right| \geq \alpha^{\prime} m \geq \alpha^{\prime} 2^{n} .
$$

Since $n^{r}$ bounds the lengths of $f^{\prime}$ 's queries almost everywhere, we have

$$
T_{A\left[0 . .2^{n+1}-1\right]} \subseteq T_{\leq n^{r}}
$$


for all sufficiently large $n$ as $A\left[0 . .2^{n+1}-1\right]$ is the characteristic string of $A_{\leq n}$. Combining (5.2) and (5.3), we have

$$
\left|T_{\leq n^{r}}\right| \geq \alpha^{\prime} 2^{n}
$$

for infinitely many $n$. This implies that

$$
\left|T_{\leq n}\right| \geq 2^{n^{\frac{1}{r+1}}}
$$

for infinitely many $n$, so $T \in \operatorname{DENSE}_{\text {i.o. }}$, a contradiction. Therefore we must have $\alpha=0$.

Finally, we have

$$
\begin{aligned}
\mathcal{L}_{\text {str }}^{\log }(\pi, A) & =\limsup _{n \rightarrow \infty} \frac{\mathcal{L}_{\pi}^{\log }(A[0 . . n-1])}{n} \\
& =\limsup _{n \rightarrow \infty} \frac{\#_{1}(n) \cdot 0+\#_{2}(n) \cdot \log (k+1)+\#_{3}(n) \cdot \log \frac{k+1}{k}}{n} \\
& =\log (k+1) \limsup _{n \rightarrow \infty} \frac{\#_{2}(n)}{n}+\log \frac{k+1}{k} \limsup _{n \rightarrow \infty} \frac{\#_{3}(n)}{n} \\
& \leq \log \frac{k+1}{k} \leq \frac{1}{k \ln 2}<\frac{2}{k} .
\end{aligned}
$$

We now extend the proof of Theorem 5.42 to show that a larger class has p-dimension 0. This is a strengthening of Theorem 5.39.

Theorem 5.43. $\operatorname{dim}_{\mathrm{p}}\left(\mathrm{P}_{\mathrm{ctt}}\left(\mathrm{DENSE}^{c}\right)\right)=0$.

Proof. Assume that $A \leq_{\mathrm{ctt}}^{\mathrm{p}} D \in \mathrm{DENSE}^{c}$ via a reduction $f$ that is computable in a polynomial time bound $q$. For each $n \in \mathbb{N}$ and $w \in\{0,1\}^{*}$, define the set of strings

$$
T_{w}^{(n)}=\bigcup_{i \geq 2^{n}-1: w[i]=1} f\left(s_{i}\right)
$$

Let $k \geq 1$ and let $\epsilon=\frac{1}{k+1}$. For each $n \in \mathbb{N}$, define a predictor $\pi_{n}$ for $A$ as follows.

- If $|w| \geq 2^{n}-1$, then: 
- If $f\left(s_{|w|}\right) \subseteq T_{w}^{(n)}$, then $\pi_{n}(w, 1)=1$ and $\pi_{n}(w, 0)=0$

- Otherwise, $\pi_{n}(w, 0)=1-\epsilon$ and $\pi_{n}(w, 1)=\epsilon$.

- Otherwise, $\pi_{n}(w, 0)=\pi_{n}(w, 1)=\frac{1}{2}$.

That is, $\pi_{n}$ behaves just like the predictor from the previous proof, except that it makes no prediction on strings of length less than $n$ and ignores the queries these strings make.

Let $p(n)=n^{2}$ and let $\delta>0$. Then for infinitely many $n$, we have

$$
\left|D_{\leq q(p(n))}\right| \leq 2^{n^{\delta}}
$$

Letting $v_{n}=A\left[0 . .2^{p(n)+1}-2\right]$, the characteristic string of $A$ on $\{0,1\} \leq p(n)$, we have

$$
T_{v_{n}}^{(n)} \subseteq D_{\leq q(p(n))}
$$

for all $n$.

Fix $n$ and assume that (5.4) is true. Define a sequence $J^{(n)}$ by

$$
J^{(n)}[j]=1 \Longleftrightarrow f\left(s_{j}\right) \subseteq T_{A[0 . . j-1]}^{(n)} .
$$

As in the previous proof, we consider the following three cases for each $j$ where $2^{n}-1 \leq j<$ $2^{p(n)+1}-1$. (Here $j$ ranges over the indices for the strings of lengths $n$ through $p(n)$.)

(1) $J^{(n)}[j]=1$ : This implies that $A[j]=1$ and $\log \frac{1}{\pi_{n}(A[0 . . j-1], A[j])}=\log 1=0$.

(2) $J^{(n)}[j]=0$ and $A[j]=1$ : Then $\log \frac{1}{\pi_{n}(A[0 . . j-1], A[j])}=\log (k+1)$.

(3) $J^{(n)}[j]=0$ and $A[j]=0$ : Then $\log \frac{1}{\pi_{n}(A[0 . . j-1], A[j])}=\log \frac{k+1}{k}$.

For each $i \in\{1,2,3\}$, let $\#_{i}^{(n)}=\mid\left\{2^{n}-1 \leq j<2^{p(n)+1}-2 \mid\right.$ Case $(i)$ holds for $\left.j\right\} \mid$. Then, as in the previous proof, we have

$$
\left|T_{v_{n}}^{(n)}\right| \geq \#_{2}^{(n)} .
$$


Combining (5.6), (5.5), and (5.4), we have

$$
\#_{2}^{(n)} \leq 2^{n^{\delta}}
$$

Now we can upper bound the log-loss of $\pi_{n}$ on $v_{n}$ whenever (5.4) holds:

$$
\begin{aligned}
\mathcal{L}^{\log }\left(\pi_{n}, v_{n}\right) & =\left(2^{n}-1\right) \cdot \log \frac{1}{2}+\#_{1}^{(n)} \cdot 0+\#_{2}^{(n)} \cdot \log (k+1)+\#_{3}^{(n)} \cdot \log \frac{k+1}{k} \\
& \leq 2^{n}+2^{n^{\delta}} \log (k+1)+\left|v_{n}\right| \cdot \log \frac{k+1}{k} \\
& \leq \frac{2}{k}\left|v_{n}\right|+2^{n}+2^{n^{\delta}} .
\end{aligned}
$$

As $\pi_{n}(w, b)$ is computable in $O\left(|w|^{2}\right)$ time, Proposition 5.40 yields a predictor $\pi_{A}$ computable in $O\left(|w|^{4}\right)$ time such that for all $n$ where (5.4) holds,

$$
\frac{\mathcal{L}^{\log }\left(\pi_{A}, v_{n}\right)}{\left|v_{n}\right|} \leq \frac{2}{k}+\frac{2^{n}+2^{n^{\delta}}+c(n+1)}{2^{n^{2}+1}-1}
$$

This happens infinitely often, so $\mathcal{L}^{\log }\left(\pi_{A}\right) \leq \frac{2}{k}$.

Proposition 5.41 implies that

$$
\operatorname{dim}_{\mathrm{p}}\left(\mathrm{P}_{\mathrm{ctt}}\left(\mathrm{DENSE}^{\mathrm{c}}\right)\right) \leq \frac{2}{k}
$$

as $A$ is an arbitrary member of the class. This holds for all $k \geq 1$, so the theorem follows.

Theorem 5.43 can be immediately extended using the work done in Section 5.1.4.

Corollary 5.44. $\operatorname{dim}_{\mathrm{p}}\left(\mathrm{P}_{1-\mathrm{tt}}\left(\mathrm{P}_{\mathrm{ctt}}\left(\mathrm{DENSE}^{c}\right)\right)\right)=0$.

Proof. By Theorem 5.17 we know that $\mathrm{P}_{1-\mathrm{tt}}\left(\mathrm{P}_{\mathrm{ctt}}\left(\mathrm{DENSE}^{c}\right)\right)$ and $\mathrm{P}_{\mathrm{m}}\left(\mathrm{P}_{\mathrm{ctt}}\left(\mathrm{DENSE}^{c}\right)\right)$ have the same p-dimension. Since $\mathrm{P}_{\mathrm{m}}\left(\mathrm{P}_{\mathrm{ctt}}\left(\mathrm{DENSE}^{c}\right)\right)=\mathrm{P}_{\mathrm{ctt}}\left(\mathrm{DENSE}^{c}\right)$, the corollary follows from Theorem 5.43 .

We now have a new proof of the following known result about hard languages for E.

Corollary 5.45. (Watanabe [63]) Every $\leq_{\mathrm{ctt}}^{\mathrm{p}}$-hard language for $\mathrm{E}$ is dense. 
Proof. Because $\operatorname{dim}_{\mathrm{p}}(\mathrm{E})=1$ and $\mathrm{p}$-dimension is monotone, we have $\mathrm{E} \nsubseteq \mathrm{P}_{\mathrm{ctt}}\left(\mathrm{DENSE}^{c}\right)$ by Theorem 5.43. 


\section{BIBLIOGRAPHY}

[1] K. Ambos-Spies, E. Mayordomo, and X. Zheng. A comparison of weak completeness notions. In Proceedings of the Eleventh IEEE Conference on Computational Complexity, pages 171-178, 1996.

[2] K. Ambos-Spies, W. Merkle, J. Reimann, and F. Stephan. Hausdorff dimension in exponential time. In Proceedings of the 16th IEEE Conference on Computational Complexity, pages 210-217, 2001.

[3] K. Ambos-Spies, H.-C. Neis, and S. A. Terwijn. Genericity and measure for exponential time. Theoretical Computer Science, 168(1):3-19, 1996.

[4] K. Ambos-Spies, S. A. Terwijn, and X. Zheng. Resource bounded randomness and weakly complete problems. Theoretical Computer Science, 172(1-2):195-207, 1997.

[5] K. B. Athreya, J. M. Hitchcock, J. H. Lutz, and E. Mayordomo. Effective strong dimension, algorithmic information, and computational complexity. Technical Report cs.CC/0211025, Computing Research Repository, 2002.

[6] C. H. Bennett and J. Gill. Relative to a random oracle $A, \mathrm{P}^{A} \neq \mathrm{NP}^{A} \neq$ co- $\mathrm{NP}^{A}$ with probability 1. SIAM Journal on Computing, 10:96-113, 1981.

[7] R. V. Book. Comparing complexity classes. Journal of Computer and System Sciences, 9:213-229, 1974.

[8] J. M. Breutzmann and J. H. Lutz. Equivalence of measures of complexity classes. SIAM Journal on Computing, 29(1):302-326, 2000. 
[9] H. Buhrman, E. Hemaspaandra, and L. Longpré. SPARSE reduces conjunctively to TALLY. SIAM Journal on Computing, 24(4):673-681, 1995.

[10] H. Buhrman and D. van Melkebeek. Hard sets are hard to find. Journal of Computer and System Sciences, 59(2):327-345, 1999.

[11] G. J. Chaitin. A theory of program size formally identical to information theory. Journal of the Association for Computing Machinery, 22:329-340, 1975.

[12] T. Cover. Universal gambling schemes and the complexity measures of Kolmogorov and Chaitin. Technical Report 12, Stanford University Department of Statistics, October 1974 .

[13] K. Falconer. Fractal Geometry: Mathematical Foundations and Applications. John Wiley \& Sons, 1990.

[14] S. A. Fenner. Gales and supergales are equivalent for defining constructive Hausdorff dimension. Technical Report cs.CC/0208044, Computing Research Repository, 2002.

[15] L. Fortnow and J. H. Lutz. Prediction and dimension. Journal of Computer and System Sciences. To appear. Preliminary version appeared in Proceedings of the 15th Annual Conference on Computational Learning Theory, pages 380-395, 2002.

[16] J. Håstad. Some optimal inapproximability results. In Proceedings of the Twenty-Ninth Annual ACM Symposium on Theory of Computing, pages 1-10, 1997.

[17] F. Hausdorff. Dimension und äusseres Mass. Mathematische Annalen, 79:157-179, 1919.

[18] J. M. Hitchcock. Correspondence principles for effective dimensions. Theory of Computing Systems. To appear. Preliminary version appeared in Proceedings of the 29th International Colloquium on Automata, Languages, and Programming, pages 561-571, 2002.

[19] J. M. Hitchcock. MAX3SAT is exponentially hard to approximate if NP has positive dimension. Theoretical Computer Science, 289(1):861-869, 2002. 
[20] J. M. Hitchcock. Fractal dimension and logarithmic loss unpredictability. Theoretical Computer Science, 304(1-3):431-441, 2003.

[21] J. M. Hitchcock. Gales suffice for constructive dimension. Information Processing Letters, 86(1):9-12, 2003.

[22] J. M. Hitchcock. Small spans in scaled dimension. Technical Report cs.CC/0304030, Computing Research Repository, 2003.

[23] J. M. Hitchcock, J. H. Lutz, and E. Mayordomo. Scaled dimension and nonuniform complexity. Journal of Computer and System Sciences. To appear. Preliminary version appeared in Proceedings of the 30th International Colloquium on Automata, Languages, and Programming, pages 278-290, 2003.

[24] J. M. Hitchcock, J. H. Lutz, and S. A. Terwijn. The arithmetical complexity of dimension and randomness. In Proceedings of the 12th Annual Conference of the European Association for Compter Science Logic, 2003. To appear.

[25] D. W. Juedes and J. H. Lutz. The complexity and distribution of hard problems. SIAM Journal on Computing, 24(2):279-295, 1995.

[26] H. Ki and T. Linton. Normal numbers and subsets of $\mathbb{N}$ with given densities. Fundamenta Mathematicae, 144:163-179, 1994.

[27] P. Lévy. Théorie de l'Addition des Variables Aleatoires. Gauthier-Villars, 1937 (second edition 1954).

[28] M. Li and P. M. B. Vitányi. An Introduction to Kolmogorov Complexity and its Applications. Springer-Verlag, Berlin, 1997. Second Edition.

[29] W. Lindner. On the polynomial time bounded measure of one-truth-table degrees and p-selectivity, 1993. Diplomarbeit, Technische Universität Berlin. 
[30] J. H. Lutz. Dimension in complexity classes. SIAM Journal on Computing. To appear. Preliminary version appeared in Proceedings of the Fifteenth Annual IEEE Conference on Computational Complexity, pages 158-169, 2000.

[31] J. H. Lutz. The dimensions of individual strings and sequences. Information and Computation. To appear. Preliminary version appeared as [35].

[32] J. H. Lutz. An upward measure separation theorem. Theoretical Computer Science, 81(1):127-135, 1991.

[33] J. H. Lutz. Almost everywhere high nonuniform complexity. Journal of Computer and System Sciences, 44(2):220-258, 1992.

[34] J. H. Lutz. A small span theorem for P/Poly-Turing reductions. In Proceedings of the Tenth Annual Structure in Complexity Theory Conference, pages 324-330, 1995.

[35] J. H. Lutz. Gales and the constructive dimension of individual sequences. In Proceedings of the 27th International Colloquium on Automata, Languages, and Programming, pages 902-913, 2000. Revised as [31].

[36] J. H. Lutz. Information and computation seminar. Iowa State University, 2000. Unpublished lectures.

[37] J. H. Lutz and E. Mayordomo. Measure, stochasticity, and the density of hard languages. SIAM Journal on Computing, 23(4):762-779, 1994.

[38] J. H. Lutz and E. Mayordomo. Cook versus Karp-Levin: Separating completeness notions if NP is not small. Theoretical Computer Science, 164(1-2):141-163, 1996.

[39] J. H. Lutz and Y. Zhao. The density of weakly complete problems under adaptive reductions. SIAM Journal on Computing, 30(4):1197-1210, 2000.

[40] P. Martin-Löf. The definition of random sequences. Information and Control, 9:602-619, 1966. 
[41] P. Mattila and R. Mauldin. Measure and dimension functions: measurability and densities. Mathematical Proceedings of the Cambridge Philosophical Society, 121:81-100, 1997.

[42] E. Mayordomo. Almost every set in exponential time is P-bi-immune. Theoretical Computer Science, 136(2):487-506, 1994.

[43] E. Mayordomo. A Kolmogorov complexity characterization of constructive Hausdorff dimension. Information Processing Letters, 84(1):1-3, 2002.

[44] N. Merhav and M. Feder. Universal prediction. IEEE Transactions on Information Theory, 44(6):2124-2147, 1998.

[45] N. Nisan and A. Wigderson. Hardness vs randomness. Journal of Computer and System Sciences, 49:149-167, 1994.

[46] P. Odifreddi. Classical recursion theory, volume 125 of Studies in Logic and the Foundations of Mathematics. North-Holland, 1989.

[47] C. A. Rogers. Hausdorff Measures. Cambridge University Press, 1998. Originally published in 1970.

[48] H. Rogers, Jr. Theory of Recursive Functions and Effective Computability. McGraw Hill, New York, N.Y., 1967.

[49] B. Ryabko. The nonprobabilistic approach to learning the best prediction. Technical Report TR02-011, Electronic Colloquium on Computational Complexity, 2002.

[50] B. Ya. Ryabko. Algorithmic approach to the prediction problem. Problems of Information Transmission, 29:186-193, 1993.

[51] B. Ya. Ryabko. The complexity and effectiveness of prediction problems. Journal of Complexity, 10:281-295, 1994.

[52] C. P. Schnorr. A unified approach to the definition of random sequences. Mathematical Systems Theory, 5:246-258, 1971. 
[53] C. P. Schnorr. Zufälligkeit und Wahrscheinlichkeit. Lecture Notes in Mathematics, 218, 1971.

[54] C. P. Schnorr. Process complexity and effective random tests. Journal of Computer and System Sciences, 7:376-388, 1973.

[55] C. P. Schnorr. A survey of the theory of random sequences. In R. E. Butts and J. Hintikka, editors, Basic Problems in Methodology and Linguistics, pages 193-210. D. Reidel, 1977.

[56] L. Staiger. Kolmogorov complexity and Hausdorff dimension. Information and Computation, 103:159-94, 1993.

[57] L. Staiger. A tight upper bound on Kolmogorov complexity and uniformly optimal prediction. Theory of Computing Systems, 31:215-29, 1998.

[58] L. Staiger. How much can you win when your adversary is handicapped? In Numbers, Information and Complexity, pages 403-412. Kluwer, 2000.

[59] D. Sullivan. Entropy, Hausdorff measures old and new, and limit sets of geometrically finite Kleinian groups. Acta Mathematica, 153:259-277, 1984.

[60] K. Tadaki. A generalization of Chaitin's halting probability $\omega$ and halting self-similar sets. Hokkaido Mathematical Journal, 31:219-253, 2002.

[61] C. Tricot. Two definitions of fractional dimension. Mathematical Proceedings of the Cambridge Philosophical Society, 91:57-74, 1982.

[62] J. Ville. Étude Critique de la Notion de Collectif. Gauthier-Villars, Paris, 1939.

[63] O. Watanabe. Polynomial time reducibility to a set of small density. In Proceedings of the Second Structure in Complexity Theory Conference, pages 138-146, 1987.

[64] A. K. Zvonkin and L. A. Levin. The complexity of finite objects and the development of the concepts of information and randomness by means of the theory of algorithms. Russian Mathematical Surveys, 25:83-124, 1970. 